DOE/ET/32079--2

DE87 010466

\title{
A LONG TERM RADIOLOGICAL RISK MODEL FOR PLUTONIUM-FUELED AND FISSION REACTOR \\ SPACE NUCLEAR SYSTEM
}

\author{
Prepared for \\ office of Coordination and Special Projects \\ U.S. Department of Energy
}

Prepared by

Bart W. Bartram

David $R$. Dougherty

\author{
NUS Corporation \\ 4 Research Place \\ Rockville, Maryland 20850
}

\section{DISCLAIMER}

This report was prepared as an account of work sponsored by an agency of the United States Government. Neither the United States Government nor any agency thereof, nor any of their employees, makes any warranty, express or implied, or assumes any legal liability or responsibility for the accuracy, completeness, or usefulness of any information, apparatus, product, or process disclosed, or represents that its use would not infringe privately owned rights. Reference herein to any specific commercial product, process, or service by trade name, trademark, manufacturer, or otherwise does not necessarily constitute or imply its endorsement, recommendation, or favoring by the United States Government or any agency thereof. The views and opinions of authors expressed herein do not necessarily state or reflect those of the United States Government or any agency thereof. 


\section{DISCLAIMER}

This report was prepared as an account of work sponsored by an agency of the United States Government. Neither the United States Government nor any agency Thereof, nor any of their employees, makes any warranty, express or implied, or assumes any legal liability or responsibility for the accuracy, completeness, or usefulness of any information, apparatus, product, or process disclosed, or represents that its use would not infringe privately owned rights. Reference herein to any specific commercial product, process, or service by trade name, trademark, manufacturer, or otherwise does not necessarily constitute or imply its endorsement, recommendation, or favoring by the United States Government or any agency thereof. The views and opinions of authors expressed herein do not necessarily state or reflect those of the United States Government or any agency thereof. 


\section{DISCLAIMER}

Portions of this document may be illegible in electronic image products. Images are produced from the best available original document. 
TABLE OF CONTENTS

Section page

1.0 INTRODUCTION 1-1

2.0 SUMMARY OF RISR III MODEL (STAGE TWO) 2-1

3.0 SUMMARY OF RISK III MODEL (STAGE ONE) 3-1

3.1 Source Term Definition 3-1

3.2 Environmental Radioactive Concentrations 3-1

3.2.1 Eigh Altitude Vapor Releases 3-1

3.2.2 High Altitude Particulate Releases 3-2

3.2.3 Low Altitude Releases 3-2

3.2.4 Aquatic Releases 3-2

3.2.5 Environmental Concentration Outputs 3-3

3.3 Radiological Dose Calculations 3-3

3.3.1 Radiological Doses (Dispersed Fuel) 3-3

3.3.2 Radiological Doses (Intact Sources) 3-4

3.4 Radiological Bealth Effects 3-4

3.5 Estimation of Risk. 3-5

4.0 RADIOISOTOPIC INVENTORY VERSUS TIME 4-1

5.0 ENVIRONMENTAL TRANSFER PROCESSES 5-I

5.1 Atmospheric Transfer Processes 5-1

5.1.1 High Altitude Releases 5-1

5.1 .1 .1 Worldwide Interaction
Statistics

5.1.1.2 High Altitude Release (Vapor) 5-6

5.1.1.3 High Altitude Particulate. Releases

5.1.2 Low Altitude Release Model

5.1.2.1 Maximum Individual Exposures $5-34$

5.1.2.2 General Population Exposures 5-38

5.2 Aquatic Dispersion Models 5-43

6.0 RADIOLOGICAL DOSE MODELS 6-I

6.1 Radiological Dose Models for Dispersed Fuel 6-1

6.2 Radiological Dose Model for Intact Fuel 6-17

7.0 POPULATION HEALTH EFFECTS MODEL 7-1

7.1 Dose Response Models 7-1

7.2 BEIR III Report Summary 7-1

8.0 UPDATE ON INTERNAL DOSE MODEL $8-1$

9.0 REFERENCES 


\section{LIST OF TABLES}

Table

Page

5-1 Equal Area Latitude Band Boundaries

$5-4$

5-2 Earth Surface Characteristics of Each

Equal Area Latitude Band

$5-5$

5-3 Fraction of Source Falling in Each Equal

Area Latitude Band as a Function of the

Injection Latitude

$5-16$

5-5 Aerodynamic Regimes for Given Particle Size Ranges

$5-28$

5-6 Worldwide Average Joint Frequency Distribution of Wind Speed and stability Class

6-1 Dose Calculations Biotransfer Factors

$6-4$

$6-2$

Usage Rates for Various Exposure Pathways

$6-7$

6-3

Dose Calculations Dose Factor Library

$6-8$

Alternative Health Effects Risk Estimators

7-1 Alternative Health Effec
for Radiation Exposure

$7-4$

$7-2$

BEIR III Cancer Risk Estimates - Fatal Cancers per Miliion Man-Rem of Total Body Exposure

$7-10$

7-3 Excess Cancer Incidence and Mortality per

Million Persons per Year per Rad by Cancer Site (Organ) 
LIST OF FIGURES

Eigure No.

$\underline{\text { Page }}$

$1-1$

Plutonium Environmental Transport

Flow Diagram

$1-2$

$2-1$

Quantified Plutonium Environmental

Transport Flow Diagram

$2-5$

$2-2$

Environmental Transfer Matrix, $\left[\lambda_{i}, j\right]$

and Its Physical Interpretation

$2-7$

4-1 Radioactive Decay of Pu-238 and

Pu-242

$4-3$

$4-2$

Radioactive Decay of Pu-239

4-4

4-3

Radioactive Decay of Pu-236 and

Pu-240

$4-5$

4-4

Radioactive Decay of $\mathrm{Pu}-241$

4-6

5-1

Cell Framework Numbering Sequence

$5-3$

$5-2$

Nomenclature for Great Circle Routes

$5-7$

$5-3$

Random Impact Probabilities for

Great Circle Routes Defined by

Inclination $\eta$

$5-8$

5-4 Expected Population Density Impacted for

Great Circle Routes Defined by Inclina-

tion $\eta$ (Land Impact Probability $=1.0$ )

$5-10$

$5-5$

High Altitude Release Parameters

for Each Atmospheric Compartment

$5-10$

5-6 Ground Level Normalized Concentrations

for High Altitude Vapor Releases

(Uniform Worldwide Distribution)

$5-14$

5-7 Deposition of Small Particles $(\leq 4 \mu \mathrm{m})$

Released at High Altitude as a Function

of Injection Latitude

$5-15$

5-8 Normalized Population Risk Factor as a Function of Injection Latitude or High Altitude Vapor Releases

Time Integrated Ground Level Airborne

Mass Concentration for Discrete Time

Periods Due to Bigh Altitude Vapor

Releases

5-24 


\section{LIST OF FIGURES (Continued)}

Figure No.

$\underline{\text { Page }}$

$5-10$

Ground Concentrations Due to High

Altitude Vapor Releases

$5-24$

5-11 Vegetation Concentrations Due to $\mathrm{High}$

Altitude Vapor Releases

$5-26$

5-12 Descent Time for Particulates Released

at High Altitudes as a Function of

Release Height and Particle Size

$5-30$

5-13 Cumulative Mass Distribution for Particles

Less Than or Equal to 2000 Microns

$5-31$

5-14 Normalized Deposition Density as a

Function of Affected Area for Various

Release Beights of $45^{\circ}$ Latitude

$5-32$

5-15 Expected Wind Speed for Random Impacts Along Great Circle Routes Defined by $\eta$

$5-35$

$5-16$

Atmospheric Stability Class for Random Impacts Along Great Circle Routes

Defined by $\eta$

$5-39$

5-17 Integrated Ground Level Airborne Concentrations Due to Low Altitude Releases

5-18 Ground Concentrations Due to Low

Altitude Releases

$5-41$

5-19 Vegetation Concentrations Due to Low

Altitude Releases

$5-41$

Dissolution of Encapsulated Fuel in

Aquatic Environments

$5-45$

5-21 Dissolution of Encapsulated Fuel in

Aquatic Enironments as a Function of

Time and Dissolution Rate

$7-1$

Comparison of Computational Methods for

BEIR III Risk Estimates

7-9

8-1 Pu-238 Inhalation Doses Bone Using

INHALE III and ICRP 19 Region Fraction for $\mu$ with Flat Particle size Distribution 


\section{LIST OF FIGURES (Continued)}

Figure No.

Page

8-2

Pu-238 Inhalation Doses Bone Using

INHALE III and ICRP 19 Region Fraction

for $\mu$, with $1 \mu \mathrm{Ci}-\mathrm{sec} / \mathrm{m}^{3}$ or entire PPO

Particle Size Distribution $(270 \mathrm{ft} / \mathrm{sec}$

Impact)

$8-4$ 


\subsection{INTRODUCTION}

Nuclear heat sources can be used in a spectrum of space, terrestrial, and undersea applications to generate electrical power by thermoelectric or dynamic-cycle conversion. The principal application is the use of plutonium (Pu) -238 in radioisotopic thermal electric generators in space nuclear electric power systems. Fission reactors also are being considered for future applications in such systems. Such systems under certain normal operating and postulated accident conditions could release activity into the environment, resulting in risks to the population in the form of radiological doses and associated health effects. This report addresses the long term modeling of such activity released into the environment and the calculation of associated radiological risk to the population.

There are many possible scenarios for the release of activity, its dispersion through the environment, and subsequent population exposure by various pathways. Activity could be released at high and low altitudes, on the land surface, or underwater. The released activity would then be dispersed into various environmental media (air, soil, and water) and interact with people through various exposure pathweys leading to inhalation, ingestion, and external radiological doses and associzted health effects. Possible scenarios for the release of activity, its dispersion in the environment, and subsequent population exposure addressed in this report are shown in the flow diagram presented in Figure 1-1. (1)

The evaluation of the radiological impact of postulated scenarios involving releases of activity into the environment includes the following steps:

1. Identification of postulated accident or normal operation release modes, including the probability of release and the release location.

2. Source term definition, including the activity of each radionuclide released and the corresponding chemical form and particle size distribution.

3. Analysis of the time behavior and dispersion of the released activity to determine the concentrations in environmental media (air, soil, and water) as a function of time.

4. Analysis of the interaction between the environmental concentrations and man, leading to ingestion, inhalation and external doses through each environmental exposure pathway.

5. The evaluation of the radiological impact on man in terms of the population doses received and the resulting health effects. 


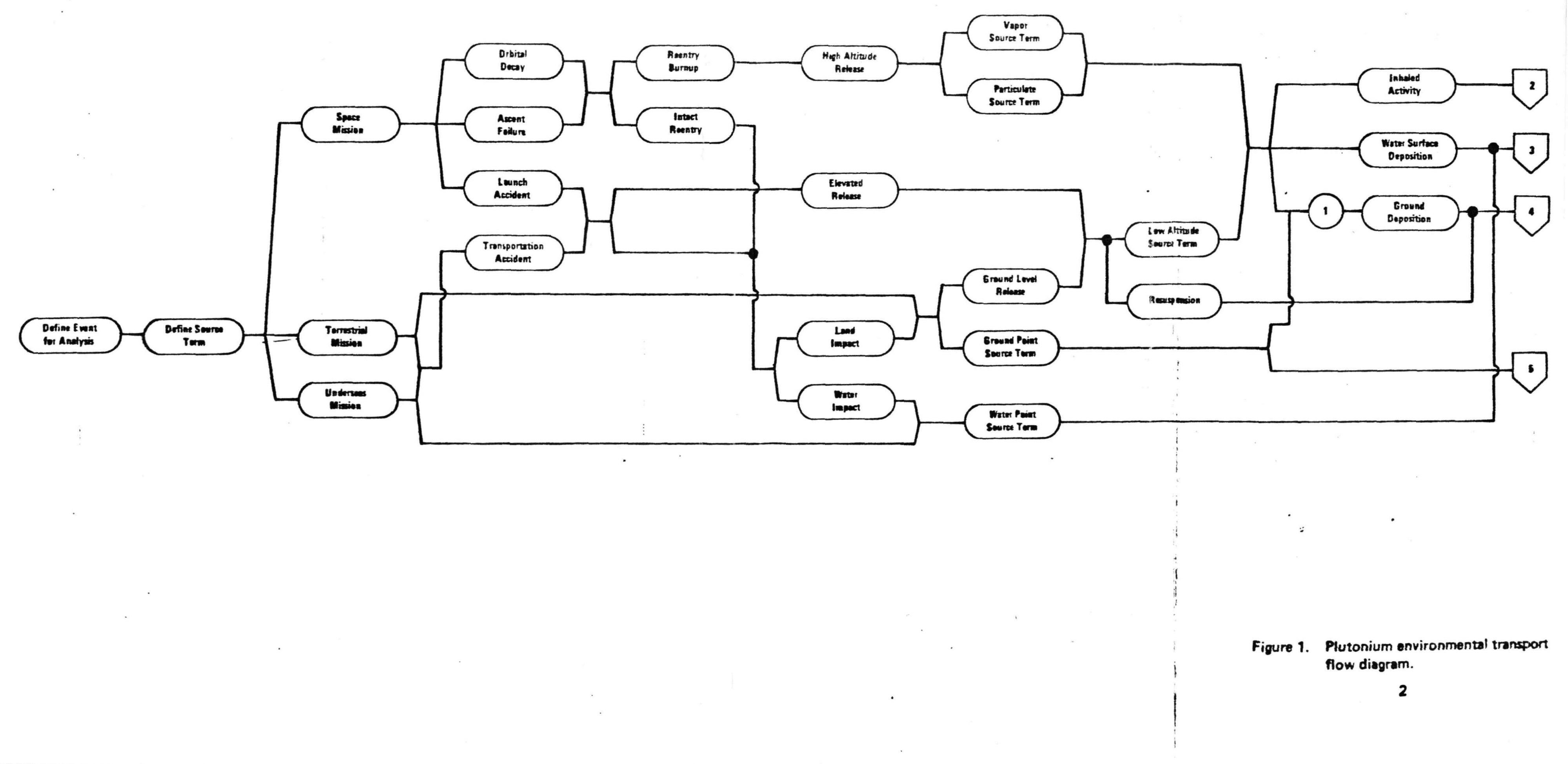




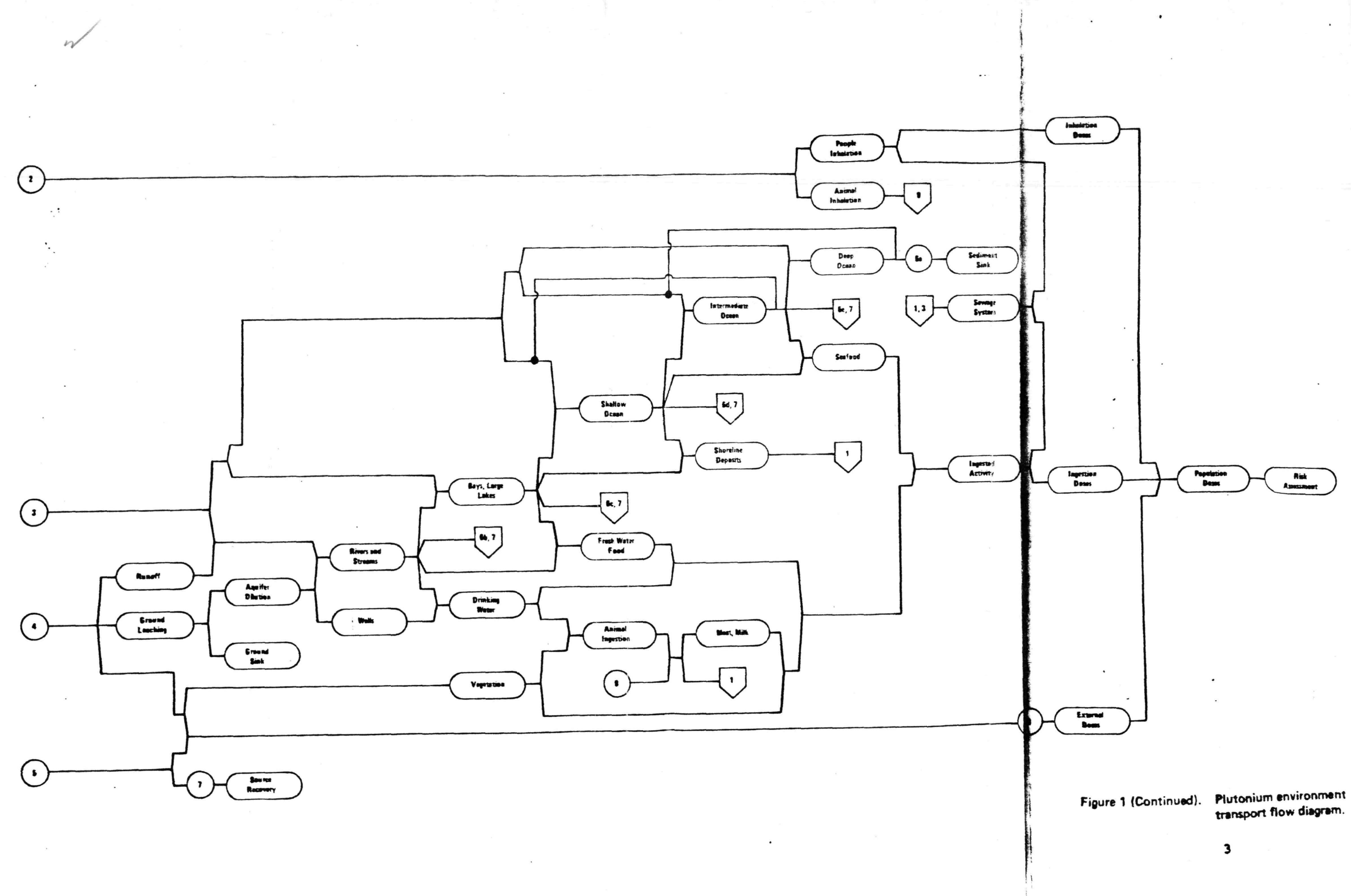


The radiological doses to the general population following a postulated release of activity can be calculated in various levels of detail depending on the amount and quality of input data available, the geographic area affected by the release, and the time scale of interest. For time scales on the order of 1 year, in which the released activity is restricted to a well-defined localized geographical area detailed dose calculations can be performed to determine the maximum individual dose, the population-versus-dose distribution, and the number of persons receiving a dose above a specified level for each environmental pathway leading to inhalation, ingestion, and external doses. In order to carry out such calculations, spatial distributions of the time-integrated activity in the air, water, and on land relative to population and food supply distributions must be determined using refined atmospheric and aquatic dispersion models. This type of calculation scheme is incorporated into the RISK II model, developed by the NUS Corporation which was designed primarily fof evaluating Pu-238 systems and is documented in OSM Volume 2.72$)$

The problem addressed in this report is the long-term risk due to activity released into the environment. The exposure is calculated in terms of the integrated population-versus-dose distribution expressed in total person-rems so that the corresponding health effects can be calculated. The time scale of interest is on the order of 1,000 of years. Because of the time scale of the model, the time transformation by radioactive decay of the fuel radionuclides must be accounted for. Furthermore, many of the environmental pathways neglected in the RISK II model must now be considered because of the time scale of the model. The model described in this report is called RISR III. It is applicable to not only Pu-238, but also fission reactor fuels and any other radioisotopic fuel mix.

The RISK III model under development is both more complex in some ways and more simplified in others than RISR II. The RISR III model is more complex in that more pathways are addressed over longer time periods and radioisotopic decay is accounted for. Due to the long term nature of the model it is somewhat more abstract. While RISR II uses wheels to cescribe localized distributions of population and other parameters, the RISR III model uses average densities. It is also assumed that a linear dose response model relates radiological doses to associated health effects. These assumptions lead to a considerable simplification in the radiological dose calculations. Conventional environmental dispersion modeling as in RISR II is required only if it is desired to calculate the dose-rate distribution over the population. Since the objective of RISR III is to calculate population exposure in terms of person-rems and apply the linear dose response model to determine health effects, a considerable simplification in the calculations results. In an air environment, for example, the critical points of initial contamination are people and food crops or animals. If it is assumed that the critical points of initial contamination are uniformly distributed throughout the environmental medium of interest, then the exposure is directly proportional to 
the total quantity of activitu in the dispersing medium and independent of the distribution of the activity in that dispersing medium. This is a direct result of the linear dose response model and the mean value theorem of integral calculus.

In the RISR III model, reflected by stage two in the development plan, the environmental mass transfer of released activity along the pathways shown in Figure $1-1$ can be represented by a set of simultaneous differential equations. The differential equations are approximated by their finite difference form. These are then put into a matrix form. An environmental response matrix is constructed where each element of the matrix represents the transfer from one environmental location to another per unit time. The quantity of activity in each environmental compartment at a given time is determined by stepping an initial environmental inventory matrix describing the scenario to be analyzed through successive time steps by successive multiplication of the environmental response matrix. Radioactive decay and radiological doses and associated health effects are incorporated in additional matrices that multiply the environmental inventory matrix. This method is known as a Markov type chain method.

The RISR III model as described in this report actually represents the first stage of a two stage model development plan. The ultimate RISR III model design, represented by stage two in the development plan, will be described below as well as in Chapter 2 to provide a framework. The present implementation of the RISR III model, representing stage one in the development plan, will be described in the chapters subsequent to Chapter 2 along with a computer program called TDOS, designed to assist in the RISR III calculations. The two stages of the development plan are described briefly below.

Many pathways shown in Figure 1-1 have been included that some may feel are insignificant. Indeed, many are insignificant in terms of individual doses. However, it is not at all clear that these same pathways are insignificant in terms of population doses over Iong time periods. Instead of prejudging and possibly deleting pathways at this point, these pathways have been retained so that the model can evaluate their significance in a more sensitive manner. Many of these pathways have also been retained because the model could be extended to other radioisotopes and toxic substances in general that may not be radioactive. This point should be kept in mind as the model is being described.

The RISK III model reflected by stage two of the development plan is fully described in Reference 1 and Chapter 2 of this report. The main limitation of RISR III as reflected in stage two of the development plan is the lack of data on many of the environmental transfer parameters, making it difficult to use in practical applications. The stage two version of RISR III represents a future model that will be userul when enough data becomes available through experimental programs to determine all the environmental transfer parameters. 
The RISK III model as reflected in stage one of the development plan represents the implementation of the long-term risk analysis methodology for practical applications. Concentrations of released fuel as a function of time in environmental media are calculated for selected release scenarios. Then using a computer code called TDOs the concentrations are converted to dose and health effects as a function of time. The RISR III model as reflected in stage one of the development plan is described in the chapters subsequent to Chapter 2 of this report.

To be realistic, the limitations of the RISR III models as well as models of a similar nature must be fully recognized. Many input parameters are required in the modeling. The values of these parameters may be wide ranging. Due to lack of data, representative values of these parameters may be poorly defined for a given scenario, and therefore somewhat arbitrarily selected. Bence the validity of the modeling results suffer. The model is intended primarily to organize an otherwise complex situation with the understanding the results of such modeling should not be considered valid in an absolute sense. The results are only as good as the input data and the extent to which the model represents the real world. With this in mind the model is considered usetul in accomplishing the following objectives:

\section{Comparing the population risk of alternative system} designs on a relative basis

2. Evaluating the relative importance of each population exposure pathway

3. Evaluating the sensitivity of the results to variations in transport parameters and system design

4. Identification of critical transport parameters requiring further experimental study.

This report describes the RISR III model in detail, including the environmental transfer of released activity, the time transformation of the initial fuel mix, the population exposure through each environmental pathway, and the resulting radiological doses and associated health effects. Chapter 2 summarizes the RISR III (Stage 2) model. Chapter 3 summarizes the RISR III (Stage 1) model. Chapter 4 describes the time transformation of the initial fuel mix due to radioactive decay. Chapter 5 develops the models needed to calculate environmental concentrations versus time. Chapter 6 describes the radiological dose models for inhaled and ingested activity, and external doses due to ground plane activity. Chapter 7 reviews the health effects associated with radiological doses and describes how such health effects are calculated. 


\subsection{SUMMARY OF RISK III MODEL (STAGE TWO)}

The chapter describes the RISt III model as reflected in stage two of the development plan. More detailed information is presented in Reference 1. The first two steps in evaluating the population risks due to the release of activity associated with a given scenario is to define the source term and evaluate its transport as a function of time throughout the environment. The source term definition includes the released mass, its chemical and isotopic composition, particle size, state of encapsulation, and release location. The initial curie activity of each isotope can then be determined from the mass quantity and isotopic composition. The initial source term is an input to the model and must be defined based on the system design and mission profile. The model describing the transport of the released source term through the environment is described in this chapter.

The transport of the released source term through the environment can be described in terms of either mass or activity. The model can be greatly simplified if source term transport in the environment is treated on a unit mass basis as a function of time and then converting the results to isotopic activity at the desired time. This results in the need for tracking only the single variable, mass, in the transport model instead of many variables represented by the spectrum of isotopes of interest and their transformations due to radioactive decay. This separation of the problem assumes that all the isotopes in the fuel mix behave chemically and physically the same in the environment.

Consider the environmental transfer flow diagram presented in Figure 1, applicable to any given source term release scenario. Mass transport of a released source term through the environment, represented in Figure 1 , can be described by a set of simultaneous time-dependent differential equations. Such a set of differential equations would be very difficult, if not impossible, to solve exactly for the quantity of mass from the initial source term that is in each location in the environment at a given time $t$. As an approximation, the mass transport problem can be treated in discrete time steps. This is equivalent to transforming the set of simultaneous differential equations into their finite difference form and solving them numerically. In this case the mass transport during time step $k$ will depend on the mass inventories at each location in the environment at time step k-1. Processes that are a function of their immediate past history are known as Markov processes, and their behavior can be described by the Markov chain techniques. (3)

The application of the Markov chain technique in this case consists of simply representing the set of finite difference equations by matrices, and stepping the solution through in discrete time steps by successive matrix multiplication. The accuracy of 
the solution increases as the time step is made smaller. A brief description of this technique is as follows:

- There are $\mathbf{N}$ mass inventory boxes or environmental locations.

- The mass inventory value for the $i^{\text {th }}$ box is designated by $x_{i}$.

- The vector $[X]^{n}$ represents the values in each box at a particular time nt. That is,

$$
\begin{aligned}
& x^{0}=x_{1}^{0}, x_{2}^{0}, \ldots, \ldots x_{i}^{0}, \ldots, x_{N}^{0} \\
& x^{1}=x_{1}^{1}, x_{2}^{1}, \ldots . . x_{i}^{1}, \ldots . ., x_{N}^{1} \\
& \text { - } \\
& \cdot \\
& x^{n}=x_{1}^{n}, x_{2}^{n}, \ldots, x_{i}^{n}, \ldots . . x_{N}^{n}
\end{aligned}
$$

The vector $[X]^{n}$ is represented by a column matrix called the environmental inventory matrix.

- The integer $\mathrm{n}$ is a time index and'represents the number of time-step intervals $\Delta t$ that have elapsed.

- The environmental transfer matrix that gives the values for inventory box $x_{i}$ after one time step is $\left[\lambda_{i}, j\right]$, where

$$
\left[\lambda_{i, j}\right]=\left[\begin{array}{ccc}
\lambda_{1,1}, \lambda_{1}, 2, \ldots, \lambda_{1, j}, \ldots, \lambda_{I, N} \\
\lambda_{2,1}, \lambda_{2,2}, \ldots, \lambda_{2, j}, \ldots, \lambda_{2, N} \\
\vdots & & \vdots \\
\lambda_{N, 1}, & \lambda_{N}, 2, \ldots, \lambda_{N, j}, \ldots, \lambda_{N, N}
\end{array}\right]
$$


where
$\lambda_{i, j}=$ fraction per unit time of the inventory quantity in box $x_{i}$ tnat is transferred into box $x_{j}$.
$\lambda_{i, i}=$ fraction per unit time of the inventory quan- tity in box $x_{i}$ that remains in box $x_{i}$ after one time step.

- The matrix multiplication to obtain new mass inventory values after one time step is as follows:

$$
[x]^{n}=[x]^{n-1}[i, j]
$$

As an example, consider an environmental pathway model with the four mass inventory boxes below. With an initial input $x_{1}$ to box $x_{1}$ and all other boxes initially at zero, the inventory of all the boxes at the end of each step will be as follows:

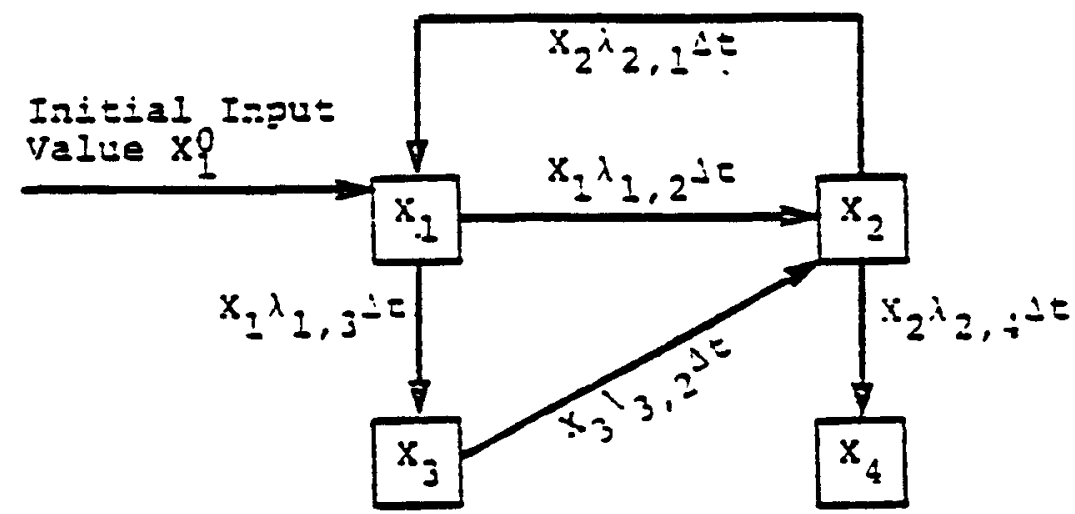

Therefore, after one time step $\Delta t$,

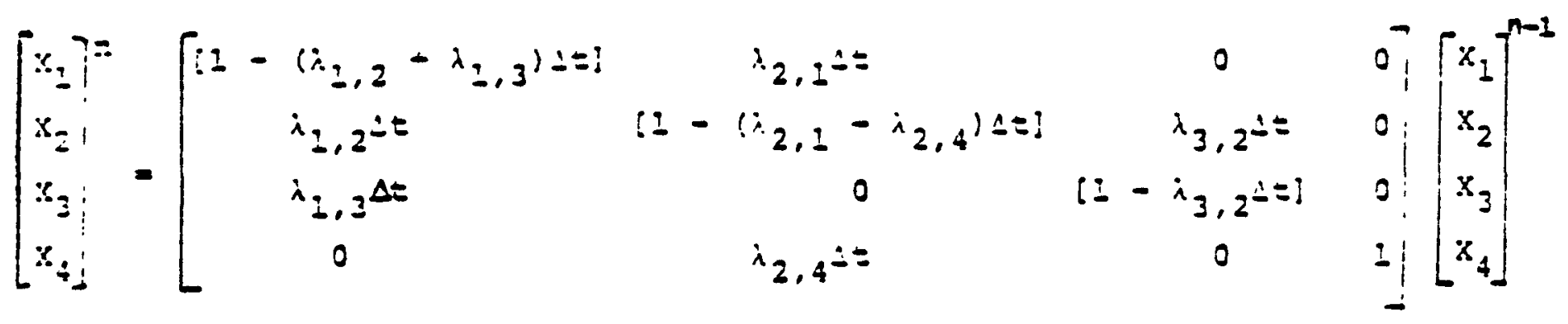

Now consider the application of the above methodology to the environmental pathway model represented by Figure 1. The elements of the environmental transfer matrix $\left[\lambda_{i} j\right]$ can be established by arbitrarily numbering the environmental locations (inventory boxes) 
shown in Figure 1-1. Eowever, the environmental transfer matrix can make more physical sense if assignments of the $i, j$ indices are made according to the type of transfer operation that occurs, including the following:

- Forward transfer pathways, $i<j$

- Feedback transfer pathways, $i>j$

- Stationary pathways, $i=j$ (occurring when a fraction of the mass remains in box $i$ after a given time step)

- Transfers to sinks

- Transfers to food products

- Transfer to man

This formulation for the assignment of $i, j$ indices has been applied to the environmental pathway diagram represented by Figure 1-1. The resulting annotated environmental pathway diagram, representing the model ardressed in this report, is presented in Figure 2-1. The corresponding environmental transfer matrix, $[\lambda i, j]$ and a diagram illustrating its physical interpretation are presented in Figure 2-2. Cetailed information describing each element of these matrices and the data inputs required for this model is presented in Appendix A.

The environmental mass transfer model thus formulated is such that at time $t=0$, initial mass source terms are assigned to environmental locations shown in Figure 2-1 appropriate to the scenario to be analyzed. This establishes the initial environmental inventory matrix, $[\mathrm{X}]$. To evaluate the mass inventory at each environmental location at time $t^{\prime}=n t$, or $n$ time steps later, represented by $[x]^{n}$, the envijonmental response matrix, $\left[\lambda_{i} j\right]$, successively multiplies $[X]$ n times. It should be noted that the elements, $\lambda_{i} j$, of the environmental response matrix, $\left[\lambda_{i}, j\right]$, describe what is happening within each mass inventory box (environmental location). The elements, $\lambda_{i}, j$, can range in complexity from constants (mass fraction per unit time transferred) to time-dependent equations in which the mass fraction transferred from a given box is a function of time.

The output from the environmental transport model at time $t$, represented by the elements of the environmental inventory matrix, $[\mathrm{X}]^{\mathrm{n}}$, is transformed into the activity associated with each isotope of the fuel mix. The elements of the environmental transfer matrix $\left[\lambda_{i}, j\right]$, described above, represent the fraction of material at location $i$ in the environment transferred to location $j$ per unit time. The elements, or environmental transfer parameters, $\lambda_{j} j$ are not necessarily constants. In general they are described by time-dependent equations representing the physical/chemical process that affects transfers from one environmental location to another. 


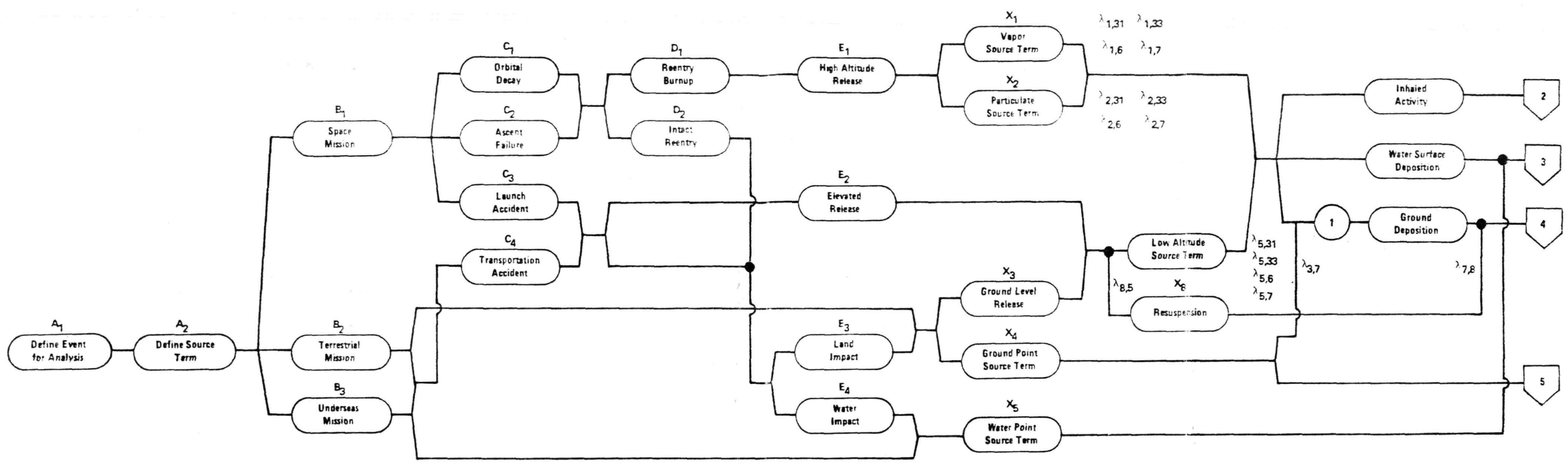




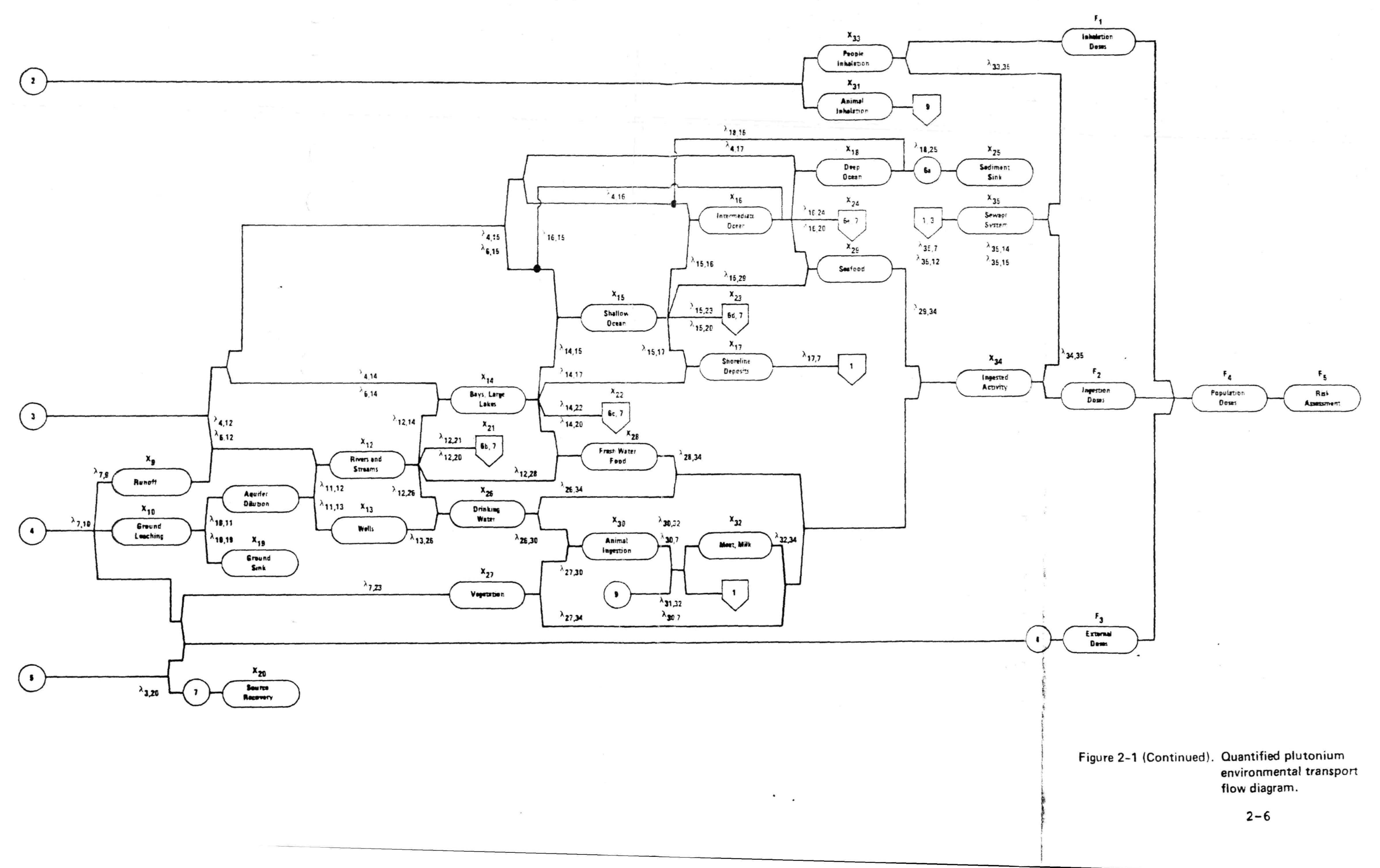



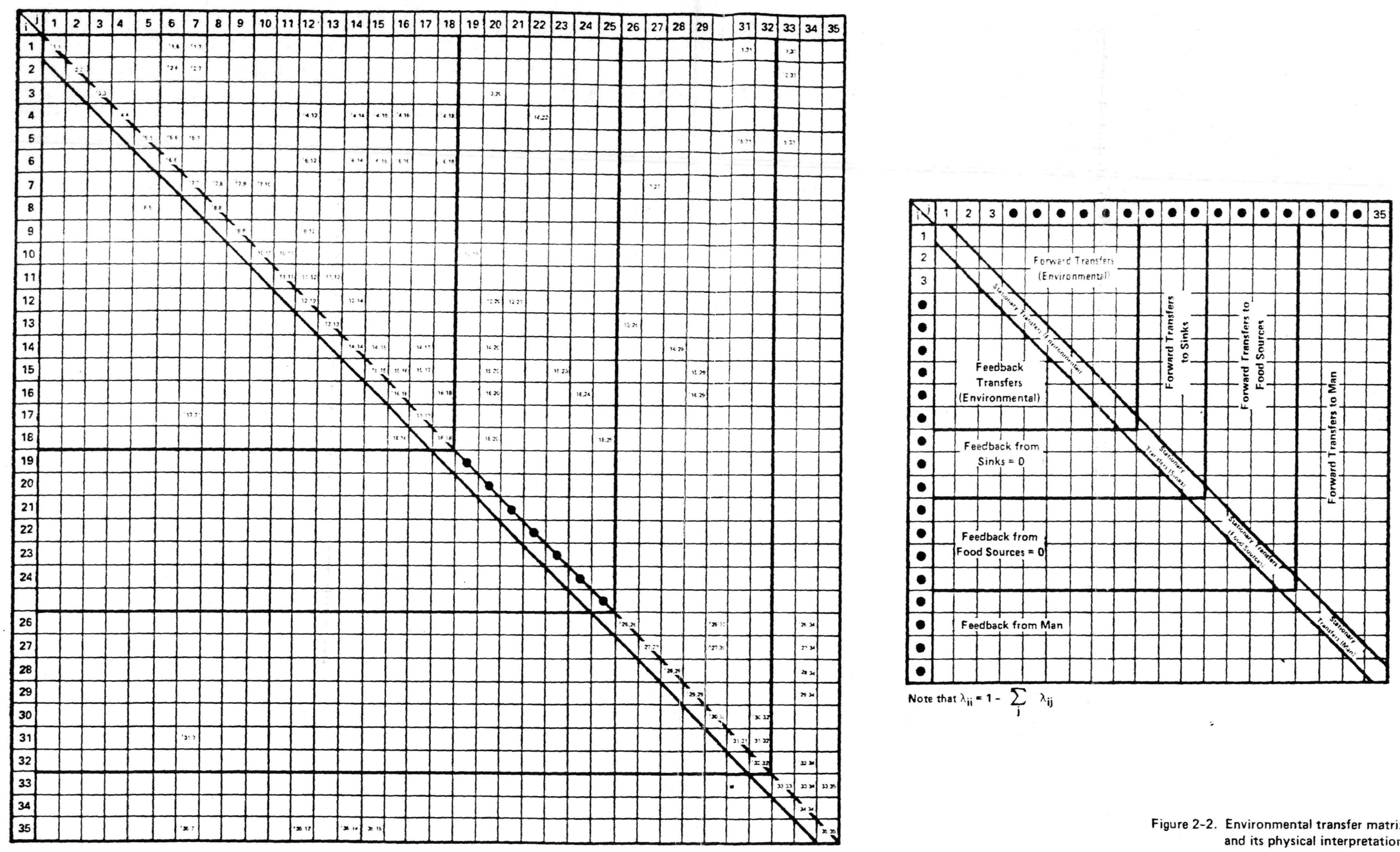

Figure 2-2. Environmental transfer matrix, $\left[\lambda_{i, j}\right]$ and its physical interpretation.

$2-7$ 
Many aspects of plutonium environmental transport are not fully understood. This is due in part to the difficulty of interpreting environmental transport in general and in part to the complicated chemical and physical properties of plutonium. Nevertheless, the large amount of data that has been collected reveals some general trends concerning environmental plutonium transport

To put these environmental transfer parameters into perspective, a brief discussion of the effects of environmental plutonium transport leading to population doses is given. Referring to Figure 2-1, the primary and secondary dose pathways are considered to be inhalation and ingestion, especially over short time periods. $(7,8)$ since plutonium generally remains in particulate form, particularly true for the more refractory oxide states $(9,10)$ such as those used in space nuclear systems, only the respirable fraction of particle sizes lead to inhalation doses. (1I) on a long-term basis, the major terrestrial and aquatic plutonium receptors are the soil and sediment. Resuspension phenomena can make the plutonium available for inhalation or deposition on plant surfaces. Plutonium dioxide has a tendency to form agglomerated particles with soil or sediment, making it less available for resuspension. $(7,11,12)$ plutonium dioxide tends to form fine particulates with weathering, leading to stronger physiochemical attachment to agglomerate paterials and further removing it from the resuspension process. Resuspension processes can af g $^{\circ}$ gntribute to the deposition of contaminants on vegetation. $(12,13)$ The larger agglomerated particles do not remain on the foliage for long periods of time.

It is generally recognized that the degree of plutonium contamination in various terrestrial or aquatic ecosystems depends strongly op the degree of intimate contact with the soil or sediment. $\{5,12,15,16,17\}$ Thus, as one progresses to the higher life forms, there is a marked decrease in plutonium concentrations.

In terrestrial ecosystems, the major pathway to people is ingestion of crops and secondarily the ingestion of animal flesh or by-products. In aquatic ecosystems, the major dose pathway to people is the ingestion of filter feeders such as mussels or clams and secondarily the ingestion of fish. Aquatic plants would contain high plutonium concentrations, but aquatic plants are usually not eaten by man. Because of the low solubility of plutonium and the purification systems for drinking water, water ingestion is a minor pathway. 


\subsection{SUMMARY OF RISR III MODEL (STAGE ONE)}

The RISR III model as reflected in stage one of the development plan is summarized in this chapter and described in detail in the subsequent chapters. The manner in which the five steps in the risk analysis procedure as described in Chapter 1 and implemented in RISR III (stage one) is summarized below.

\subsection{SOURCE TERM DEFINITION}

The specific activity of the fuel at the time of reentry has been evaluated using the ORIGIN code as described in Chapter 4.0. To account for long term behavior of released fuel in the environment, specific activities were also calculated for time steps from $10^{2}$ seconds to $10^{11}$ seconds following release.

\subsection{ENVIRONMENTAL RADIOACTIVE CONCENTRATIONS}

Environmental radioactive concentrations have been calculated as a function of time for the following types of releases:

- High altitude vapor releases

- High altitude particulate releases

- Low altitude (including ground level) releases

- Aquatic releases

In order to account for the radiological impact over long time periods, the concentrations due ts a given release were calculated in ten time steps ranging from $10^{2}$ to 1011 seconds. Furthermore, the evaluation of environmental concentrations was performed on strictly a fuel mass basis due to the large number of radionuclides of interest. Mass concentration was then converted to radioactivity concentration of each radionuclide by applying the time dependent specific activity factors generated as described in section 3.1 .

\subsubsection{HIGH ALTITUDE VAPOR RELEASES}

Eigh altitude vapor releases result from partial or complete burnup due to reentry heating. The transport of such vapor (assumed to be $45 \mu \mathrm{m}$ in diameter) is governed primarily by worldwide atmospheric circulation patterns. The atmosphere can be divided into three compartments: tne mesosphere (4.5 x $10^{4} \mathrm{~m}$ altitude), the stratosphere $\left(10^{4}-4.5 \times 10^{4} \mathrm{~m}\right)$, and the troposphere $\left(0-10^{4} \mathrm{~m}\right)$. Transport between these atmospheric compartments has been described in an approximate manner by an exponential box model, analogous to radioactive decay in $_{2} \mathrm{f}$ ich a residence half-life is assigned to each compartment. $(19,20)$ The residence half-lives are based on fallout data derived from nuclear weapons testing. Latitudinal variation of the ground level activity derived from the high altitude release at a given latitude is also based on nuclear weapons fallout data. 
For high altitude vapor releases at a given latitude, the model predicts the normalized ground plane and airborne concentrations as a function of latitude and time after release.

\subsubsection{HIGH ALTITUDE PARTICULATE RELEASES}

High altitude particulate releases ( $>45 \mu \mathrm{m}$ in diameter) can occur under the same conditions that result in high altitude vapor releases described above. The resulting particulates will follow trajectories to the earth's surface that are determined by the mass, size, and snape of each particle, the release altitude, as well as the winds and density profile of the atmosphere.

The atmospheric dispersion of such particulate released at high altitudes has been treated in Reference 3 using a modified version of the Model $B$ computer code developed by the Travelers Research Corporation. (21) In this model, the trajectories of individual mass elements are cetermined based on the rate of change of latitude, longitude and altitude during local wind speed components and the terminal fall velcocity of the particles. A particle size
distribution was used that is typical of fractured Pu- $0_{2}$ fuel, $(2)$ and assumed representative of the fuel types used in this study.

Inputting the release altitude and latitude, the model predicts the ground plane concentration as a function of affected area. Resuspension and weathering of ground plane deposition is accounted for outside of the model.

\subsubsection{LOW ALTITUDE RELEASES}

Low altitude dispersion of fuel can result from 1) ground level releases following land impact or 2) resuspension of activity previously deposited derived from both high and low altitude releases.

Radiological doses to the general population due to low altitude releases must account for vanishing small concentrations at great distances from the point of impact because such concentrations are important when a linear dose response model is used. A special model is used in calculating population dose in which all released activity is accounted for in an average concentration calculation.

\subsubsection{AQUATIC RELEASES}

Aquatic transfer processes include the interaction of airborne dispersed activity and intact sources with water bodies. These interactions include fuel dissolution, transfers to sediment, and concurrent covering of intact sources by sedimentation and encrustation.

These processes have been characterized with an exponential box model with results evaluted parametrically as a function of time required to breach encapsulation, fuel dissolution rate, transfer 
rate of dissolved fuel to sediment, and removal rate of intact sources by sedimentation or encrustation.

Average aquatic concentrations were calculated as a function of time for the ten discrete time steps mentioned previously. The average concentrations were calculated based on reference volumes

- required to produce annual $\mathrm{fish/seafood}$ and drinking water to persons within the affected fallout area, accounting for the land/ water fraction within the area.

\subsubsection{ENVIRONMENTAL CONCENTRATION OUTPUTS}

The outputs of the environmental concentration models and interaction statistics described above include the following information for ten time steps centered from $10^{2}$ to $10^{11}$ seconds following the accident:

- Normalized airborne concentration $\left(g / \mathrm{m}^{3} / g\right.$ released)

- Normalized ground concentration $\left(g / \mathrm{m}^{2} / g\right.$ released)

- Normalized vegetation concentration $\left(g / \mathrm{m}^{2}\right.$. leaf area/g released)

- Normalized aquatic concentration $\left(g / \mathrm{m}^{3} / g\right.$ released)

- Total grams of fuel released

- Number of persons affected.

These results serve as input to the radiological dose calculations described in the next section.

\subsection{RADIOLOGICAL DOSE CALCULATIONS}

Maximum individual and population doses were calculated for both dispersed fuel and intact sources as described below.

\subsubsection{RADIOLOGICAL DOSES (DISPERSED FUEL)}

Radiological doses due to dispersed fuel were calculated in discrete time steps following the release, using as inputs the results of the environmental concentration calculations described in the previous section. A special computer code, TDOS, was developed by NUS to evaluate the doses in discrete time steps using the dose methodology presented in NRC Regulatory Guide 1.109. (22) For individual and population exposures during each time step, the resulting 50-year integrated dose commitments were calculated for the following pathways:

\footnotetext{
- Inhalation

- Cloud immersion

- Ground plane radiation

- Vegetation ingestion
} 

- Meat ingestion
- Milk ingestion
- Water ingestion
- Aquatic foods ingestion.

Resulting health effects, evaluated as described in section 3.4, are also calculated by TDOS.

The Tros code output includes the following:

- User supplied input parametrics

- User supplied specific activities by nuclide and time

- Dose factor library

- Bio-transfer factors

- Dose or health effects by nuclide, time, organ and pathway

- Environmental dose commitments and health effects for the first year, first 100 years, and the total for all time periods.

\subsubsection{RADIOLOGICAL DOSES (INTACT SOURCES)}

Radiological doses due to external radiation from intact sources have been evaluated using standard methods. The intact sources were treated as point sources with radiation fields attenuated inversely as the square of the distance and by absorption. Buildup in the air media and interactions at the air/ground interface were accounted for in a limited manner.

The results were then reported in terms of rem/hr as a function of distance and time.

\subsection{RADIOLOGICAL HEALTH EFFECTS}

Health effects associated. with radiological doses resulting from dispersed fuel and intact sources include acute maximum individual health effects and long term population effects.

A linear dose response model was used in evaluating long term population health effects based on the NAS-NRC Committee on the Biological Effects of Ionizing Radiation (BEIR) as presented in the BEIR I report. (23) The linear dose response model assumes the health effects, in terms of premature cancer deaths, are proportional to tne dose, even for vanishingly small doses. A linear-quadratic dose response model was adopted by the BEIR Committee in the recently published BEIR III report, $(24)$ but it was not possible to incorporate it in the present analysis at this time. However, the BEIR III approach is fully described. The linear dose response model is considered to be conservative, especially for low dose levels, when compared to the linear quadratic model. 


\subsection{ESTIMATION OF RISR}

The overall mission risk of each system was calculated as follows:

$$
R_{i}=\sum_{j} \sum_{k} P_{i j k} \quad r_{i j k}
$$

where

$$
\begin{aligned}
R_{i}= & \begin{array}{l}
\text { overall mission risk of system } i \text {, expected fatalities/ } \\
\text { mission }
\end{array} \\
\mathbf{r}_{i j k}= & \begin{array}{l}
\text { radiological impact of system } i \text { with release type } j \\
\text { during mission phase } \mathrm{k} \text { with conditional probability } \\
\text { of } 1.0, \text { deaths }
\end{array} \\
\mathbf{P}_{i j k}= & \begin{array}{l}
\text { probability of release type } j \text { during mission phase } k \\
\text { for system } i .
\end{array}
\end{aligned}
$$




\subsection{RADIOISOTOPIC INVENTORY VERSUS TIME}

The initial radioisotopic fuel mix in plutonium-fueled space nuclear systems will be transformed in time as the initial radioisotopes decay and daughter radioisotopes accumulate. In the case of a fission reactor system, fission products are generated during operation followed by fission product decay after shutdown. The radioisotopic mix and, therefore, the radiological hazard of each system will vary over time. The radioisotopic inventory of each system has therefore, been evaluated.

The initial radioisotopic fuel $\mathrm{mix}$ and the basic characteristics of earh fuel type used in the systems under consideration are described previously in Appendix $\mathrm{H}$.

The decay or buildup of a given member of a radioactive decay chain can be described by the Bateman equation: $(25)$

$$
N_{j}=N_{i}^{0} \prod_{k=1}^{j-1} \sum_{l=1}^{j} \frac{\exp (-\lambda l t)}{\prod_{\substack{m=1 \\ m \neq l}}^{n}(\lambda m-\lambda l)}
$$

where

$$
\begin{aligned}
& \mathbf{N}_{i}^{O}= \text { number of } i \text { th parent atoms per gram of fuel at time } 0 \\
& \mathbf{N}_{j}=\text { number of } j \text { th daughter atoms per gram of fuel at time } t \\
& \mathbf{k}=\text { radioactive decay constant, sec-l }
\end{aligned}
$$

The number of atoms of a given isotope initially in the released fuel $\mathrm{mix}$ is

$$
N_{i}^{O}=\frac{P_{i} M A_{O}}{100 M_{i}}
$$

where

$$
\begin{aligned}
& M=\text { mass of fuel released, grams } \\
& P_{i}=\text { weight percent of isotope } i \text { initially in fuel } \\
& M_{i}=\text { mass of } 1 \text { gram-mole of isotope } i, g r a m s \\
& A_{0}=\text { Avogadro's number. }
\end{aligned}
$$

The activity of a given radioisotope is

$$
A_{j}=\frac{N_{j} \lambda_{j}}{a}
$$


where

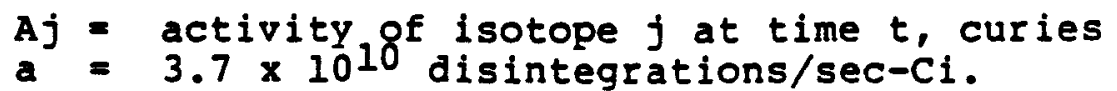

The radioisotopic inventory versus time for each fuel type and system has been calculated using the ORNL-developed ORIGEN code that uses the Bateman equation as the basis for the calculations. (18) Inputs to ORIGEN include the initial curie activity of each radioisotope in a given fuel type descrjbed in section 3.1 , and the output includes curie activity versus time for all parent and daughter radioisotopes, the gamma photon production rate and energy spectrum versus time, and the neutron production rate and energy spectrum versus time. Neutron production due to plutonium mix spontaneous fissioning and $(\alpha, n)$ reactions are accounted for.

The gamma and neutron production rates and energy spectra versus time obtained from ORIGEN are used as input to the external radiation field calculations described in Chapter 5. The radioactive decay schemes for the radionuclides in initial plutonium fuel mixes are presented in Figures 4-1 through 4-4. 

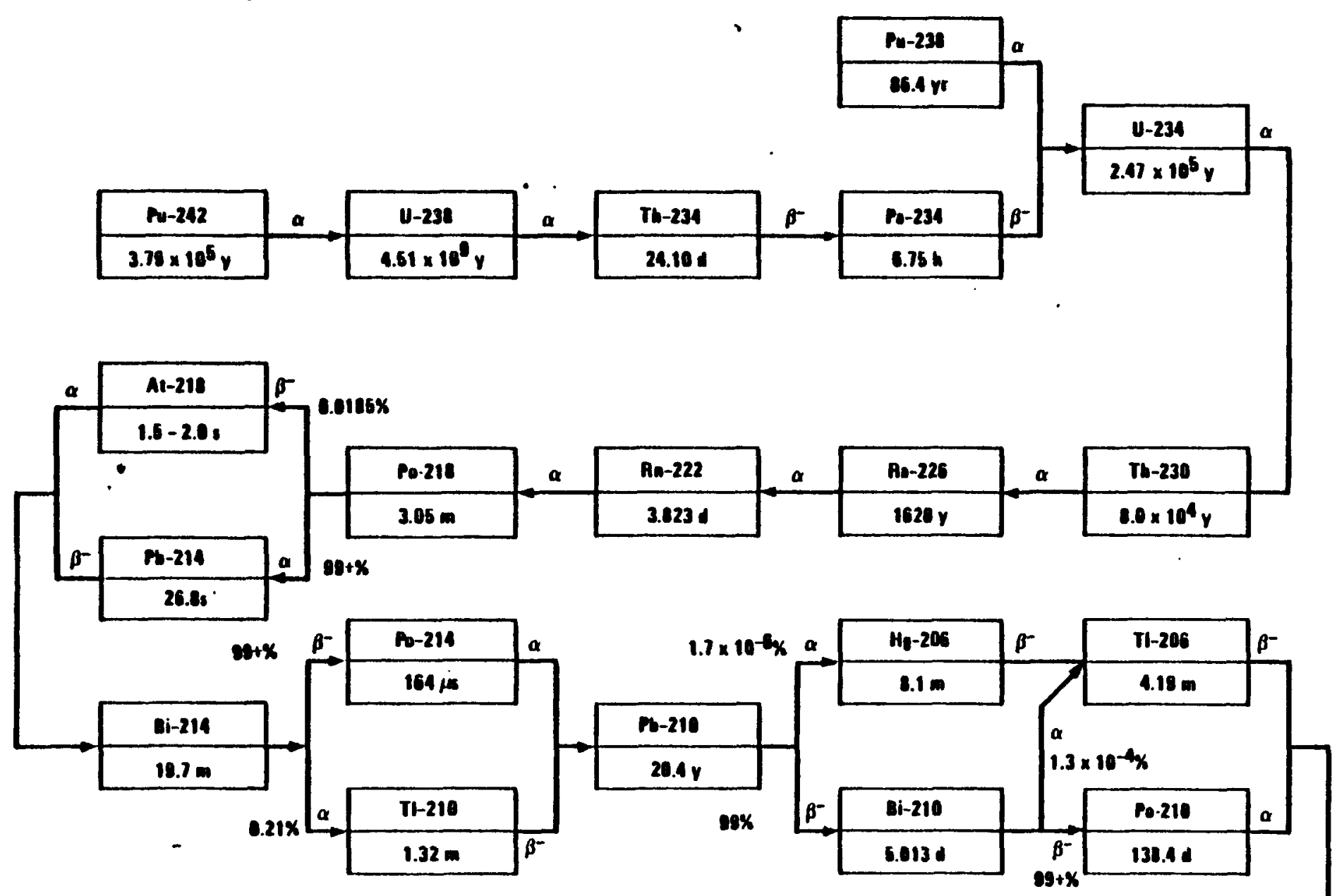

Figure 4-1. Radioactive decay of Pu-238 and Pu-242. 


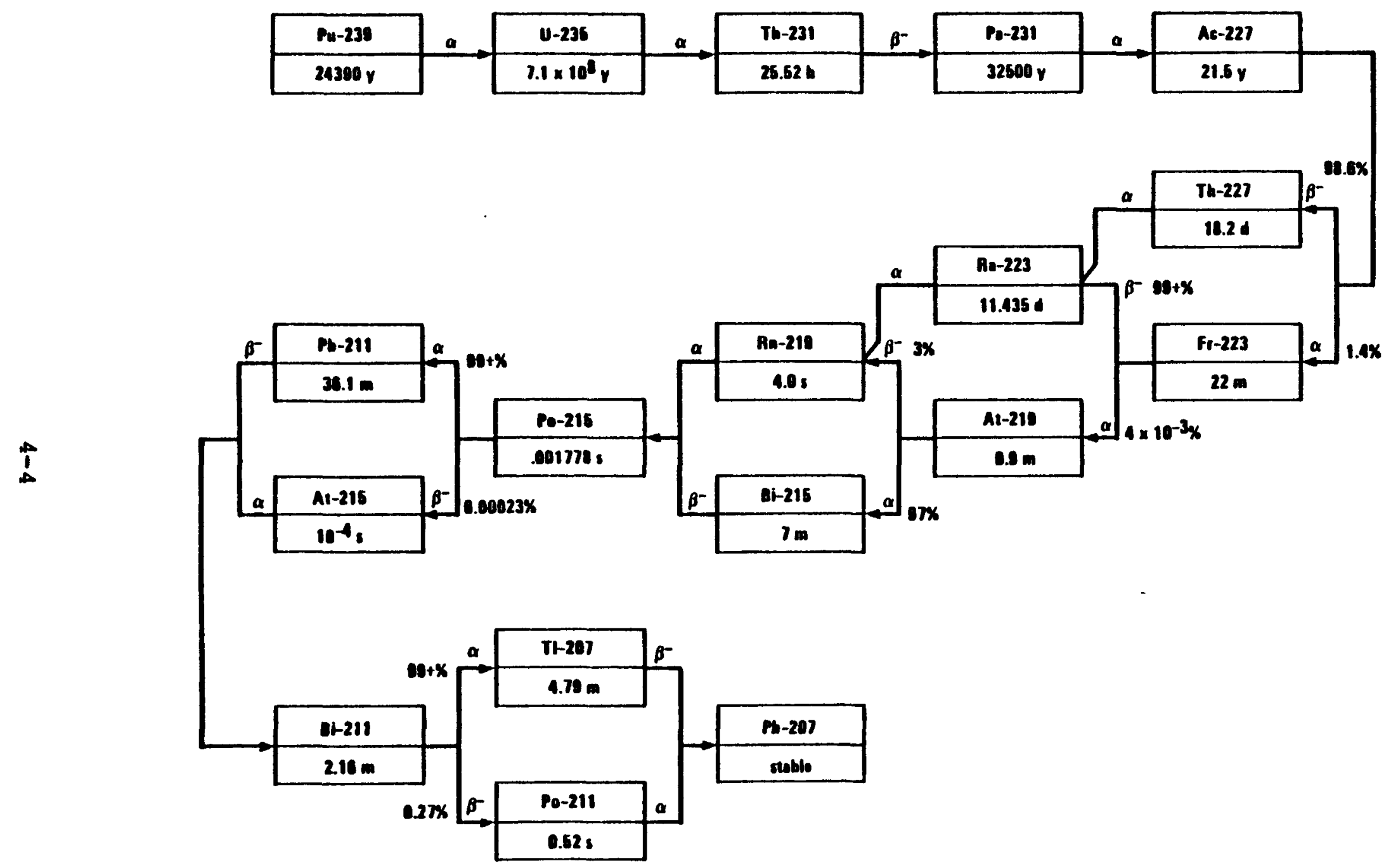

Figure 4-2. Radioactive decay of Pu-239. 


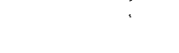

.

\begin{tabular}{|c|c|c|c|c|c|c|c|c|}
\hline$P u-240$ & $\alpha$ & $U-236$ & $a$ & Th-232 & $a$ & Ro-221 & $\beta^{-}$ & Ac-228 \\
\hline $8620 y$ & & $2.38 \times 10^{7} y$ & & $1.39 \times 10^{10} y$ & & $6.7 y$ & & $6.13 \mathrm{~h}$ \\
\hline
\end{tabular}

\begin{tabular}{|c|c|c|c|}
\hline$P u-236$ & $a$ & $u-232$ & $\alpha$ \\
\hline $2.05 y$ & & \\
\hline & & \\
\hline
\end{tabular}

\begin{tabular}{|c|c|c|c|c|c|c|c|c|c|}
\hline $\boldsymbol{\rho}$ & $P b-212$ & $a$ & Pe-216 & $a$ & $8 n-220$ & $a$ & An-224 & $\alpha$ & $T h-221$ \\
\hline & $-19.54 \mathrm{~h}$ & & 0.1458 & & 55.38 & & $3.54 \mathrm{~d}$ & & $1.91 \mathrm{y}$ \\
\hline
\end{tabular}

i

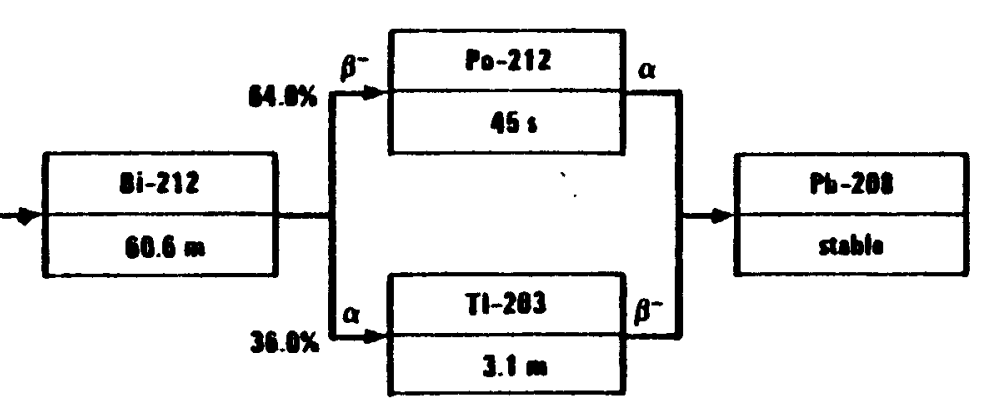

Figure 4-3. Radioactive decay of Pu-236 and Pu-240. 



\subsection{ENVIRONMENTAL TRANSFER PROCESSES}

The environmental transfer of released activity into the environment will be governed by many physical and chemical processes. These are described in this chapter for the following:

- Atmospheric transfer processes

- Aquatic transfer processes

In evaluating these environmental transfer processes, consideration must be given to interaction statistics. That is, for a given release scenario, the regional environment must be described so that the interaction between the source term and the environment can be evaluated. Interaction statistics for a given release scenario would include information such as the distribution of land, fresh water, ocean, population, and food supplies in the region that is expected to be affected by the release. General information of this nature is presented in the Overall safety Manual, Volumes 2 and 3 , for releases occurring on a worldwide basis and more specific information on releases in the vicinity of Cape Rennedy. (2) The specific interaction statistics required in a given analysis will be identified in the description of each environmental transfer process.

\subsection{ATMOSPHERIC TRANSFER PROCESSES}

Atmospheric transfer processes of interest in this study include the following:

1. High altitude release of vapor, particulate, and intact subassemblies resulting from system breakup.

2. Low altitude dispersion of elevated releases associated with an explosion or fire, and ground level releases of impacted fuel or resuspended material.

These two groups of atmospheric transfer processes are described further below.

\subsubsection{HIGH ALTITUDE RELEASES}

Each form of the released activity (vapor, particulates, or intact subassemblies) will be transported to ground level by differing mechanisms, ranging from atmospheric transport in the case of vapor to a trajectory behavior in the case of subassemblies, with particulates ranging in between depending on the mass, physical size, and shape of the released particulates. Each type of release therefore requires a different treatment in its behavior.

Before discussing each type of high altitude release in detail, consideration should first be given to interaction statistics 
relating the high altitude release point and features of the earth's surface. These interaction statistics are developed in a preliminary mariner in the next section and expanded upon in subsequent sections addressing each high altitude release type.

\subsubsection{Worldwide Interaction Statistics}

Earth surface characteristics affecting the risk of fuel released at high altitudes in the form of vapor, particulates, or intact subaşemblies have been analyzed in terms of a worldwide grid system. (2) A grid system was selected which produced 20 equal area latitude bands. Each of the 20 latitude bands was segmented into 36 equal area cells in $10^{\circ}$ longitude increments. Thus, a total of 720 cells are contained within the framework shown in Figure 5-1. Each equal area cell has an area of $708,435 \mathrm{~km}^{2}$, and its boundary latitudes are determined from

$$
A=R^{2}\left(\sin \theta_{2}-\sin \theta_{1}\right)\left(\theta_{2}-\theta_{1}\right)
$$

where

$$
\begin{aligned}
& \theta=\text { latitude } \\
& \theta=\text { longitude }
\end{aligned}
$$

The latitude bands for both the northern and southern hemisphere are bounded as shown in Table 5-1.

The grid established above allows the characteristics of each cell to be normalized to the same area. The surface characteristics of each cell, including the distribution of land, ocean, rock, soil, and population are developed in Volumes 2 and 3 of the Overall Safety Manual. (2) Ocean fractions are in turn divided into shallow ( $75 \mathrm{~m}$ average depth), intermediate $(500 \mathrm{~m}$ average depth), and deep ( $>500 \mathrm{~m}$ average depth) ocean fractions. Data on land and fresh water distribution for the onited states indicates that fresh water covers approximately 2.2 percent of the land. It has been assumed that the same fresh water fraction also characterizes the remaining land areas of the world. The resulting earth surface characteristies for each equal area latitude band is summarized in Table 5-2. This data is used to develop interaction statistics for high altitude release scenarios and earth impact scenarios as described below.

Consider a great circle earth track between two locations on earth having latitude/longitude coordinates of $\left(\theta_{1}, \phi_{1}\right)$ and $\left(\theta_{2}, \phi_{2}\right)$, respectively. The great circle route between these 


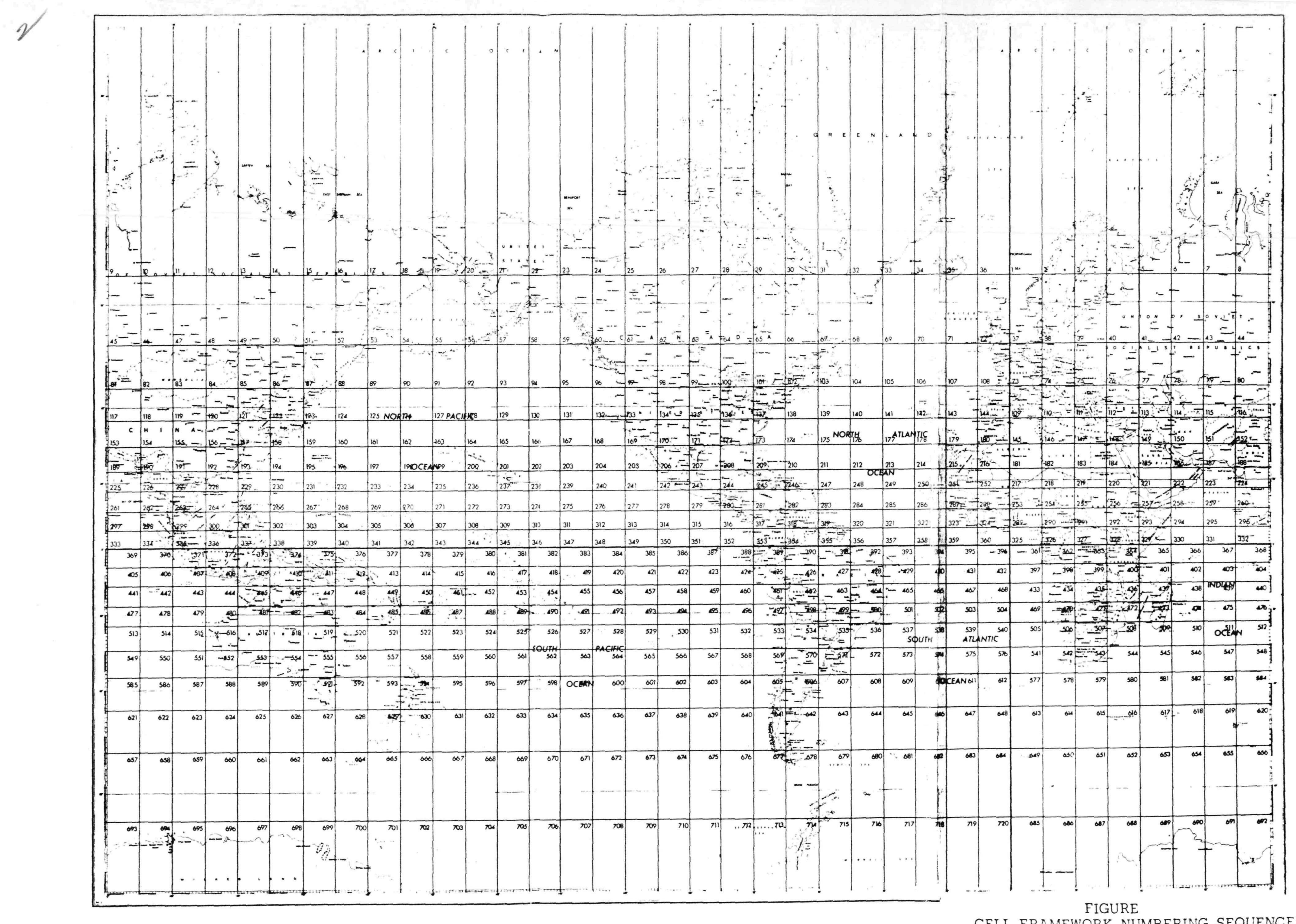


Table 5-1. Equal Area Latitude Band Boundaries

\section{Number of Band}

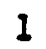

2

3

4

5

6

7

8

9

10

11

12

13

14

15

16

17

18

19

20

\section{Iatinde Rance}

$64^{\circ} 9 \cdot 30^{\prime \prime} \mathrm{N}-90^{\circ} \mathrm{N}$

$53^{\circ} 7 \cdot 30^{\prime \prime} \mathrm{N}-64^{\circ} 9.30^{\prime \prime} \mathrm{N}$

$44^{\circ} 26^{\prime} 30^{\prime \prime} \mathrm{N}-53^{\circ} 7.30^{\prime \prime} \mathrm{N}$

$36^{\circ} 52^{\prime} 30^{\prime \prime} \mathrm{N}-44^{\circ} 26^{\prime} 30^{\prime \prime} \mathrm{N}$

$30^{\circ} 30^{\prime \prime} \mathrm{N}-36^{\circ} 52 \cdot 30^{\prime \prime} \mathrm{N}$

$23^{\circ} 35^{\prime} 30^{\prime \prime} \mathrm{N}-30^{\circ} 30^{\prime \prime} \mathrm{N}$

$17^{\circ} 27^{\prime} 30^{\prime \prime} \mathrm{N}-23^{\circ} 35^{\prime} 30^{\prime \prime} \mathrm{N}$

$11^{\circ} 32^{\prime} 30^{\prime \prime} \mathrm{N}-17^{\circ} 27^{\prime} 30^{\prime \prime} \mathrm{N}$

$5^{\circ} 44^{\prime} 30^{\prime \prime} \mathrm{N}-11^{\circ} 32^{\prime} 30^{\circ} \mathrm{N}$

- Equator - $5^{\circ} 44^{\prime} 30^{\prime \prime} \mathrm{N}$

$5^{\circ} 44^{\prime} 30^{\prime \prime} \mathrm{S}$ - Equator

$11^{\circ} 32^{\prime} 30^{\prime \prime} \mathrm{s}-5^{\circ} 44^{\prime} 30^{\prime \prime} \mathrm{s}$

$17^{\circ} 27^{\prime} 30^{\prime \prime} \mathrm{S}-11^{\circ} 32 \cdot 30^{\prime \prime} \mathrm{s}$

$23^{\circ} 35^{\prime} 30^{\prime \prime} \mathrm{S}-17^{\circ} 27^{\prime} 30^{\prime \prime} \mathrm{S}$

$30^{\circ} 30^{\prime \prime} \mathrm{S}-23^{\circ} 35^{\prime} 30^{\prime \prime} \mathrm{S}$

$36^{\circ} 52.30^{\prime \prime} \mathrm{S}-30^{\circ} 30^{\prime \prime} \mathrm{s}$

$44^{\circ} 26^{\prime} 30^{\prime \prime} \mathrm{s}-36^{\circ} 52 \cdot 30^{\prime \prime} \mathrm{s}$

$53^{\circ} 730^{\prime \prime} \mathrm{s}-44^{\circ} 25^{\prime} 30^{\prime \prime} \mathrm{s}$

$64^{\circ} \mathrm{g} \cdot 30^{\prime \prime} \mathrm{s}-43^{\circ} 7130^{\prime \prime} \mathrm{s}$

$90^{\circ} \mathrm{s}$

- $64^{\circ} \mathrm{g} 30^{\prime \prime} \mathrm{s}$ 
Table 5-2. Earth Surface Characteristics of Each Equal Area Latitude Band

\begin{tabular}{|c|c|c|c|c|c|c|c|c|c|c|}
\hline $\begin{array}{l}\text { Lat I tude } \\
\text { band }\end{array}$ & $\begin{array}{c}\text { Land } \\
\text { rraction }\end{array}$ & Population & $\begin{array}{c}\text { Population } \\
\text { density. } \\
\text { persons/km2 }\end{array}$ & $\begin{array}{c}\text { soll } \\
\text { raction. }\end{array}$ & $\begin{array}{c}\text { Rock } \\
\text { Eraction }\end{array}$ & $\begin{array}{l}\text { Preab- } \\
\text { water } \\
\text { fraction } \\
\end{array}$ & $\begin{array}{c}\text { Ocean } \\
\text { fraction }\end{array}$ & $\begin{array}{c}\text { shallon } \\
\text { ocean } \\
\text { fraction }\end{array}$ & $\begin{array}{c}\text { Intermedi ate } \\
\text { ocean } \\
\text { fraction } \\
\end{array}$ & $\begin{array}{c}\text { Deep } \\
\text { ocean } \\
\text { fraction } \\
\end{array}$ \\
\hline 1 & 0.1656 & $30,169,587$ & 2.89 & 0.0 & 1.000 & 0.0102 & 0.5344 & 0.1648 & 0.1444 & 0.2252 \\
\hline 2 & 0.5850 & $161,300,755$ & 12.16 & 0.250 & 0.750 & 0.0129 & 0.1150 & 0.1247 & 0.0704 & 0.2199 \\
\hline 3 & 0.5677 & $432,222,025$ & 33.58 & 0.500 & 0.500 & 0.0124 & 0.4323 & 0.0441 & 0.0452 & 0.3430 \\
\hline 4 & 0.4548 & $543,074,832$ & 52.67 & 0.749 & 0.251 & 0.0100 & 0.5452 & 0.0349 & 0.0429 & 0.4813 \\
\hline 5 & 0.4370 & $691,722,558$ & 69.82 & 0.847 & 0.153 & 0.0096 & 0.5630 & 0.0357 & 0.0290 & 0.4983 \\
\hline 6 & 0.3971 & $605,118,210$ & 67.22 & 0.912 & 0.088 & 0.0087 & 0.6029 & 0.0312 & 0.0365 & 0.5352 \\
\hline 7 & 0.3349 & $387,278,363$ & 51.01 & 0.924 & 0.076 & 0.0074 & 0.6651 & 0.0358 & 0.0334 & 0.5959 \\
\hline 8 & 0.2522 & $199,943,660$ & 34.97 & 0.942 & 0.058 & 0.0055 & 0.7478 & 0.0214 & 0.0300 & 0.6964 \\
\hline 9 & 0.2128 & $203,931,205$ & 62.99 & 0.923 & 0.077 & 0.0031 & 0.8572 & 0.0400 & 0.0368 & 0.7801 \\
\hline 10 & 0.2150 & $78,887,239$ & 16.18 & 0.916 & 0.084 & 0.0047 & 0.7850 & 0.0400 & 0.0197 & 0.7253 \\
\hline 11 & 0.2481 & $69,355,336$ & 12.33 & 0.956 & 0.044 & 0.0055 & 0.7519 & 0.0326 & 0.0263 & 0.6930 \\
\hline 12 & 0.2189 & $132,699,175$ & 26.74 & 0.945 & 0.055 & 0.0048 & 0.7811 & 0.0387 & 0.0299 & 0.7125 \\
\hline 13 & 0.2169 & $43,233,315$ & 0.79 & 0.915 & 0.085 & 0.0048 & 0.7831 & 0.329 & 0.0200 & 0.7302 \\
\hline 14 & 0.2480 & $58,286,844$ & 10.37 & 0.911 & 0.089 & 0.0055 & 0.7520 & 0.0128 & 0.0319 & 0.7073 \\
\hline 15 & 0.2231 & $44,606,711$ & 8.02 & 0.908 & 0.092 & 0.0049 & 0.7769 & 0.0088 & 0.0155 & 0.7526 \\
\hline 16 & 0.1372 & $37,073,250$ & 12.18 & 0.888 & 0.112 & 0.0030 & 0.8628 & 0.0185 & 0.0172 & 0.8271 \\
\hline 17 & 0.0463 & $7,566,457$ & 7.21 & 0.704 & 0.296 & 0.0010 & 0.9537 & 0.0101 & 0.0256 & 0.9090 \\
\hline 10 & 0.0223 & 028,162 & 1.64 & 0.169 & 0.531 & 0.0005 & 0.9777 & 0.0172 & 0.0427 & 0.9178 \\
\hline 19 & 0.0036 & 56,396 & 0.69 & 0.235 & 0.765 & 0.0000 & 0.9964 & 0.0036 & 0.0115 & 0.9813 \\
\hline 20 & 0.5438 & 149 & 0.00 & 0.0 & 1.000 & 0.0120 & 0.4562 & 0.0077 & 0.0850 & 0.3635 \\
\hline
\end{tabular}


two locations can be defined by two angles, $\eta$ and $\phi$, shown in Figure 5-2 where

$$
\begin{aligned}
\eta & =\begin{array}{l}
\text { angle between the equatorial plane and the cir- } \\
\text { cumference plane of the great circle route }
\end{array} \\
\phi & =\begin{array}{l}
\text { longitude of the point of intersection of the } \\
\text { great circle circumference and the equator }
\end{array} \\
\theta_{1}, \theta_{2}= & \begin{array}{l}
\text { latitudes of the beginning and end of the great } \\
\text { circle route leg }
\end{array} \\
\phi_{1}, \phi_{2}= & \begin{array}{l}
\text { longitudes of the beginning and end of the great } \\
\text { circle route leg }
\end{array}
\end{aligned}
$$

For great circle routes whose circumference planes make an angle $\eta$ with the equatorial plane, impact probabilities for various earth surface characteristics have been computed in Reference 2 . The resulting impact probabilities for random impacts along a given great circle route defined by $\eta$ are summarized in Figures 5-3 and 5-4. It should be noted that $\eta$ is equivalent to an orbital inclination.

It is assumed that trajectory particulates and intact subassemblies released within a given equal area latitude band will be deposited on the earth's surface within that band, except for high altitude vapor releases which are discussed in section 5.1.1.2. When the injection latitude is known, the impact probabilities presented in Table 5-2 are used.

\subsubsection{High Altitude Release (Vapor)}

A high altitude vapor release is associated with the destruction and burnup of all or part of the system following a planned or unplanned system destruction. The transport of such vapor (assumed to be $\leq 4 \mu \mathrm{m}$ in diameter) is governed primarily by worldwide atmospheric circulation patterns. The atmosphere can be divided into three compartments: the mesosphere $\left(>4.5 \times 10^{4} \mathrm{~m}\right.$ altitude), the stratosphere $\left(10^{4}-4.5 \times 10^{4} \mathrm{~m}\right)$, and the troposphere $\left(0-10^{4} \mathrm{~m}\right)$. Transport between these atmospheric compartments can be described in an approximate manner by an exponential box model, analogous to radioactive decay, in which activity within a given compartment has a resident half-life. Studies based primarily on fallout from nuclear weapons testing indicate the regidept half-lives presented in Figure 5-5 for each compartment. $(1,19,20)$

The quantity of activity released in the mesosphere that is in each compartment after time $t$ is: 
1

Figure 5-2. Nomenclature for graat circle routes.

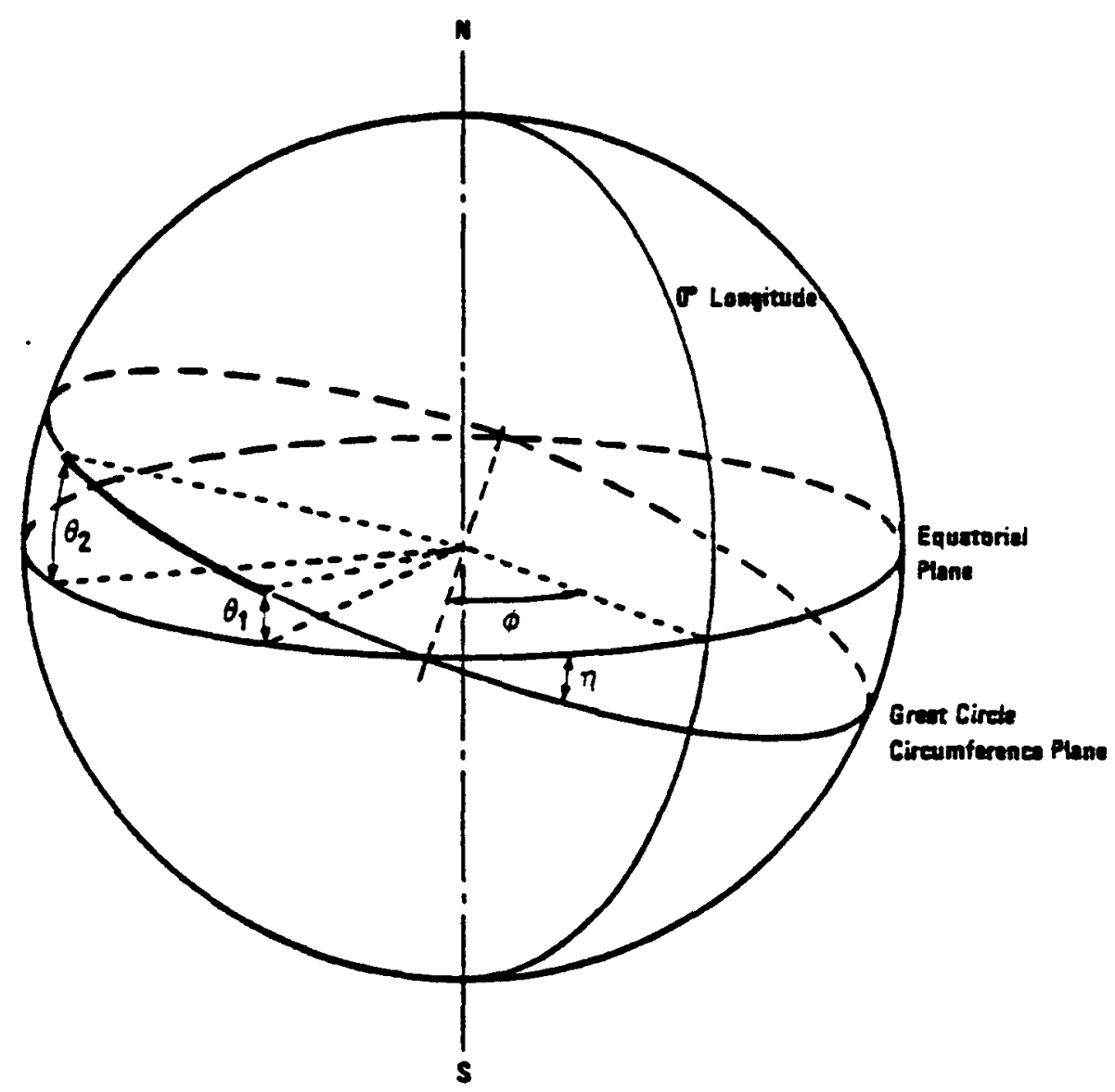




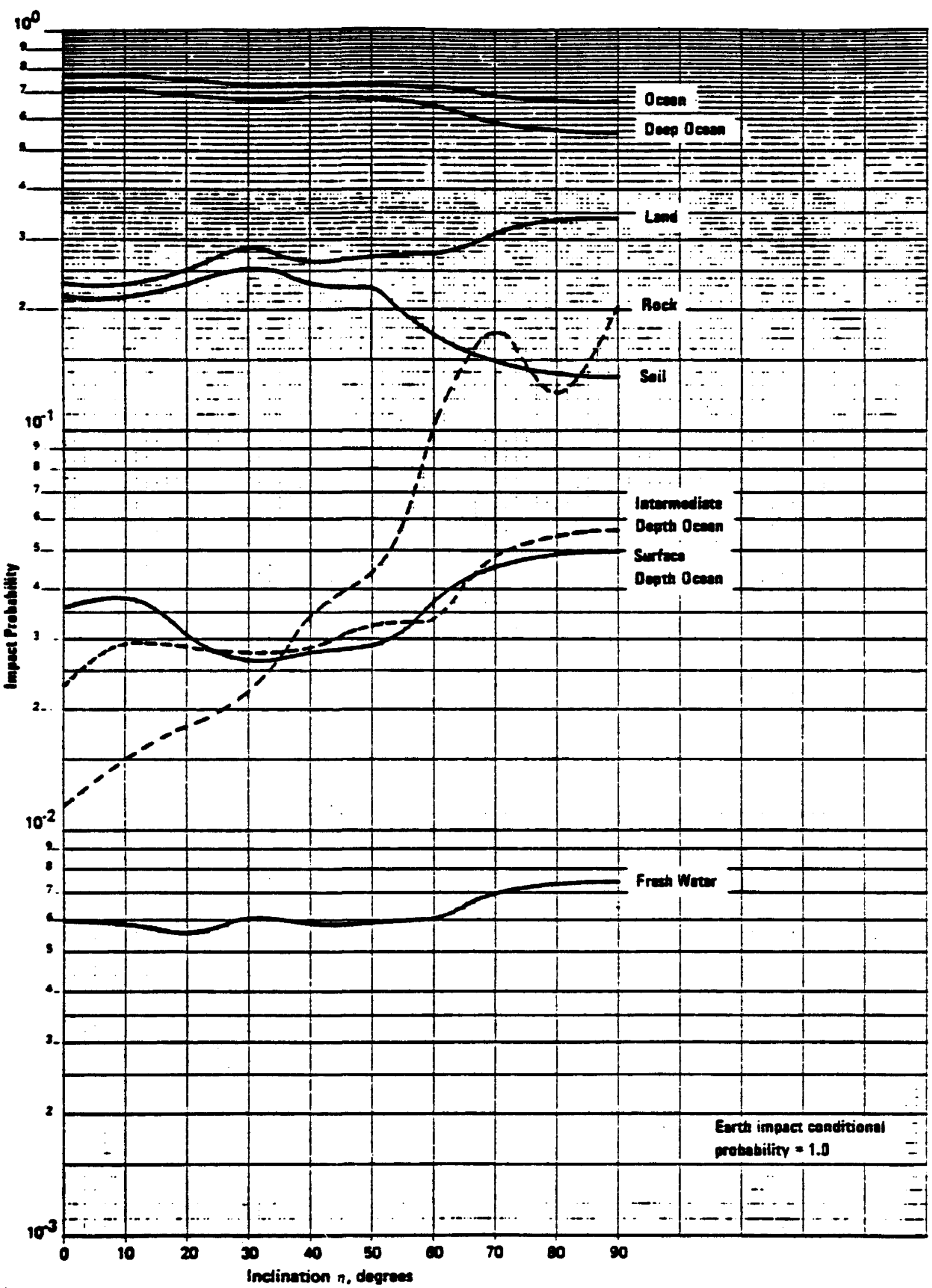

Figure 5-3. Random impact probabilities for great circle routes defined by inclination $\eta$. 


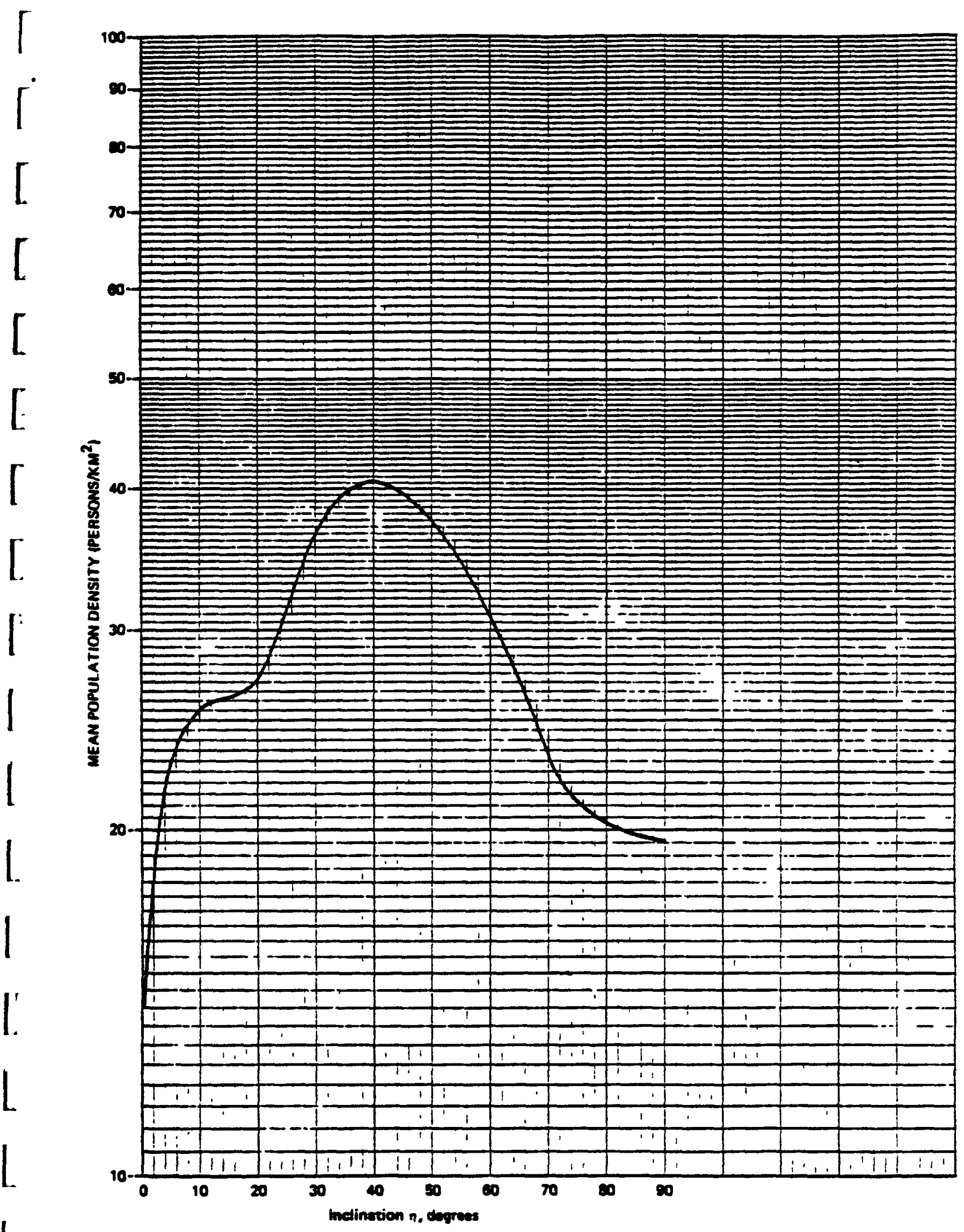

Figure 5-4. Expected population density impacted for great circle routes defined by inclination $n$ (land impact probability $=1.0$ ). 


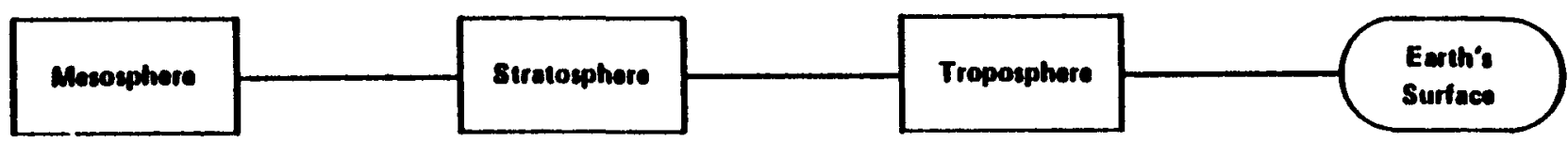

\begin{tabular}{|c|c|c|c|}
\hline Alliteude & $>4.6 \times 10^{4} \mathrm{~m}$ & $10^{4}-4.5 \times 10^{4} \mathrm{~m}$ & $0-10^{4} \mathrm{~m}$ \\
\hline $\begin{array}{l}\text { Residence } \\
\text { Hall-Life }\end{array}$ & 5 years & $0.76-2$ yoors & $5-15$ days \\
\hline $\begin{array}{l}\text { Pesidance } \\
\text { Hall-Life } \\
\text { Assumed in } \\
\text { This sindy }\end{array}$ & 6 years & 1.1 vears & 10 days \\
\hline
\end{tabular}

Figure 6-5. High altitude release parameters for each atmospheric compartment. 
Mesosphere: $\quad Q_{\mathrm{m}}(t)=Q_{\mathrm{m}}(0) \mathrm{e}^{-\lambda_{1} t}$

Stratosphere: $\quad Q_{s}(t)=Q_{m}(0) \frac{\lambda_{1}}{\lambda_{2} \lambda_{1}}\left(e^{-\lambda_{1} t-e^{-\lambda_{2} t}}\right)$

Troposphere:

$(5-4)$

$Q_{I}(t)=Q_{m}(0) \lambda_{1} \lambda_{2}\left[\frac{e^{-\lambda_{1} t}}{\left(\lambda_{3}-\lambda_{1}\right)\left(\lambda_{2}-\lambda_{1}\right)}+\frac{e^{-\lambda_{2} t}}{\left(\lambda_{3}-\lambda_{2}\right)\left(\lambda_{1}-\lambda_{2}\right)}+\frac{e^{-\lambda_{2}}=}{\left(\lambda_{1}-\lambda_{2}\right)\left(\lambda_{2}-\lambda_{3}\right)}\right]$

Earth's Surface:

$$
\begin{aligned}
Q_{\Xi}(t)=Q_{m}(0)[1 & +\frac{\lambda_{2} \lambda_{3}}{\left(\lambda_{1}-\lambda_{2}\right)\left(\lambda_{3}-\lambda_{1}\right)} e^{-\lambda_{1} t}+\frac{\lambda_{1} \lambda_{3}}{\left(\lambda_{2}-\lambda_{1}\right)\left(\lambda_{3}-\lambda_{2}\right)} e^{-\lambda_{2} t} \\
& \left.+\frac{\lambda_{1} \lambda_{2}}{\left(\lambda_{3}-\lambda_{1}\right)\left(\lambda_{2}-\lambda_{3}\right)} e^{-\lambda_{3} t}\right]
\end{aligned}
$$

where

$$
\begin{aligned}
Q_{m}(0)= & \begin{array}{l}
\text { Initial fuel source term released in the mesosphere, } \\
\text { grams }
\end{array} \\
Q_{m}(t)= & \text { Quantity of fuel in mesosphere after time } t \text {, grams } \\
Q_{s}(t)= & \begin{array}{l}
\text { Quantity of fuel in stratosphere after time } t, \\
\text { grams }
\end{array} \\
Q_{t}(t)= & \text { Quantity of fuel in troposphere after time } t \text {, grams } \\
\lambda_{1}= & \text { Eraction of fuel in mesosphere transferred to } \\
& \text { stratosphere per unit time } \\
\lambda_{2}= & \text { Eraction of fuel in stratosphere transferred to } \\
& \text { troposphere per unit time } \\
\lambda_{3}= & \text { fraction of fuel in troposphere transferred to } \\
& \text { earth's surface per unit time }
\end{aligned}
$$

It should be noted that

$$
\lambda_{i}=\frac{0.693}{T_{i} 1 / 2}
$$


where

$\lambda=$ transfer factor for atmospheric compartment $i, y^{-1}$

$T_{i l / 2}=\underset{i, y r}{\text { residence half-life of atmospheric compartment }}$

The quantity of activity in each compartment at time $t$ due to releases in the stratosphere is

$$
\begin{array}{ll}
\text { Stratosphere: } & Q_{s}(t)=Q_{s}(0) e^{-\lambda_{2} t} \\
\text { Troposphere: } & Q_{\frac{5}{5}}(t)=Q_{s}(0) \cdot\left(\frac{-\lambda_{2}}{\lambda_{3}-\lambda_{2}}\right)\left(e^{-\lambda_{2} t}-e^{-\lambda_{3} t}\right)
\end{array}
$$

Earth's Surface:

$$
\theta_{E}(t)=\theta_{5}(0)\left[I+\left(\frac{\lambda_{3}}{\lambda_{2}-\lambda_{3}}\right) e^{-\lambda_{2} t}+\left(\frac{\lambda_{2}}{\lambda_{3}-\lambda_{2}}\right) e^{-\lambda_{3} t}\right]
$$

The quantity of activity in each compartment at time $t$ due to releases in the troposphere is

$$
\begin{array}{ll}
\text { Troposphere: } & Q_{T}(t)=Q_{T}(0) e^{-\lambda_{3} t} \\
\text { Earth's surface: } & Q_{E}(t)=Q_{T}(0)\left(I-e^{-\lambda_{3} t}\right)
\end{array}
$$

The concentration in the troposphere at a given altitude is assumed to be proportional to the air density. The atmospheric density can be approximated by

$$
p(h)=a e^{-b h}
$$

where

$$
\begin{aligned}
p(\mathrm{~h}) & =\text { air density at altitude } \mathrm{h}, \mathrm{kg} / \mathrm{m}^{3} \\
\mathrm{a} & =1.3 \mathrm{~kg} / \mathrm{m}^{3} \mathrm{~m} \mathrm{~m}^{-1} \\
\mathrm{~b} & =1.283 \times 10^{4} \mathrm{~m}^{-1}
\end{aligned}
$$

Integrating. $p(h)$ from $h=0$ to $h=10^{4} \mathrm{~m}$, the mass of the troposphere is

$$
M_{T}=3.74 \times 1018 \mathrm{~kg}
$$


Assuming uniform worldwide distribution, the concentration within the troposphere is

$$
\bar{x}(h, t)=\frac{g(t)}{L_{T}} a e^{-b h}
$$

At ground level, $h=0$ and

$$
\bar{x}(0, t)=\frac{Q_{T}(t)}{M_{T}} \mathbf{a}
$$

The normalized concentration is

$$
\frac{\bar{x}(0, t)}{Q(0)}=\frac{Q_{T}(t)}{Q(0)} \frac{a}{Q_{T}}
$$

where

$$
Q(0)=\text { initial source term, grams }
$$

In the troposphere and the stratosphere, where the removal halflives have been given in Figure $5-5$ as a range, it is assumed the balf-life within a given compartment varies linearly with altitude over the half-life range for that compartment. Calculations were made of the ground level normalized concentrations due to releases at varying altitudes using Equations $(5-4),(5-8)$ and $(5-10)$. The results are presented in Figure 5-6.

For a given high altitude release it is also important to evaluate the distribution of material on the earth surface after deposition, since this will be highly non-uniform due to worldwide atmospheric dispersion patterns. Based on fallout data following nuclear weapons testing in the upper atmosphere, a fallout pattern presented in Figure 5-7 is expected. $(2,19,20)$ The distribution is assumed to be Gaussian in each hemisphere with a minimum occurring at the equator and maximums at mid-latitudes. The actual distribution will depend on the injection latitude or the latitude where reentry burnup occurs. Deposition of released vapor on the earth's surface has been evaluated as a function of injection latitude based on Figure 5-7. The results are sumarized in Table 5-3 as a function of injection latitude in terms of the fraction deposited in each of 20 equal area latitude bands described in section 5.1.1.1. The fraction of vapor released at high altitude transferred to each earth surface type is 


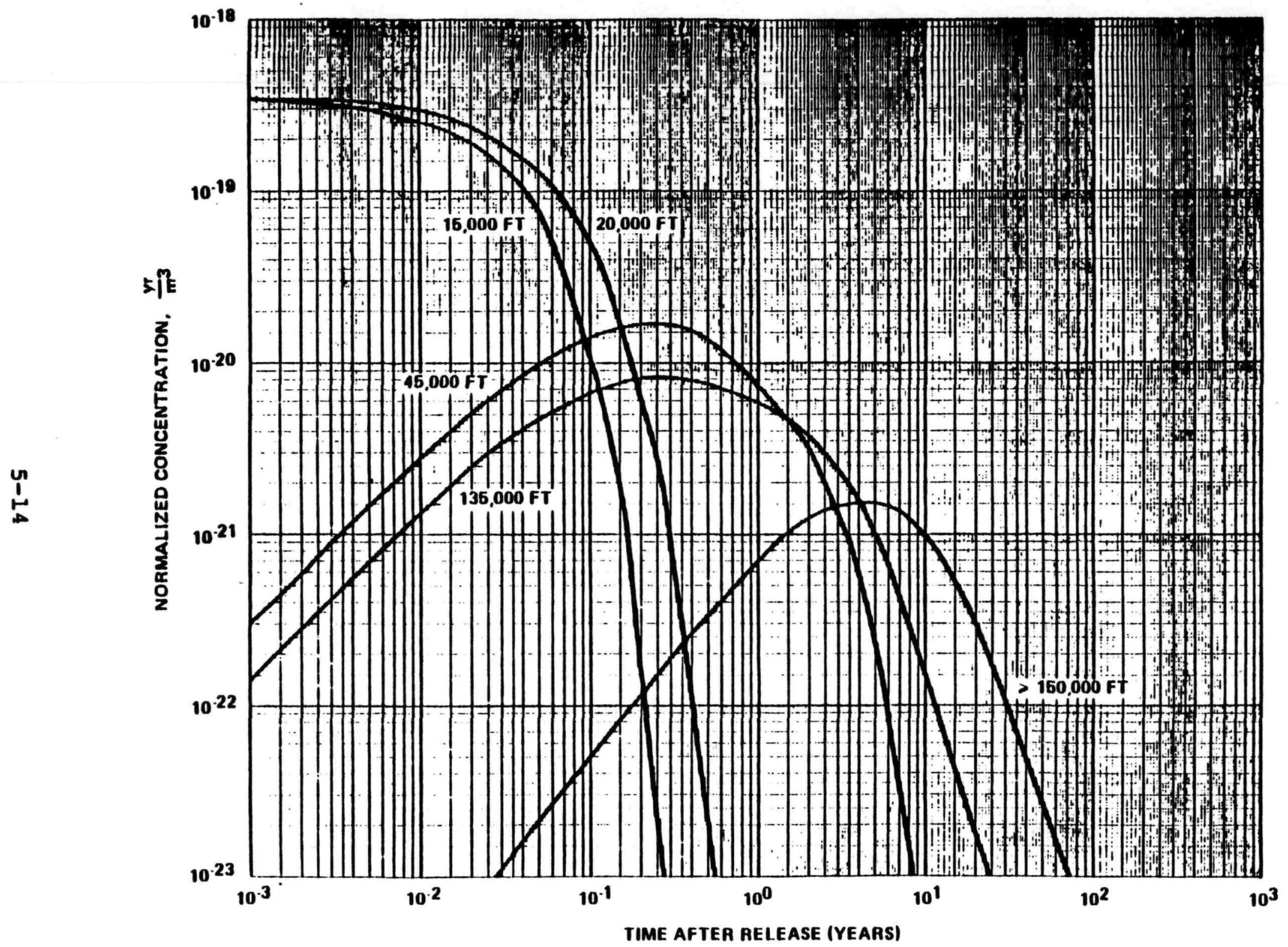

Figure 5-6. Ground level normalized concentrations for high altitude vapor releases (uniform worldwide distribution). 


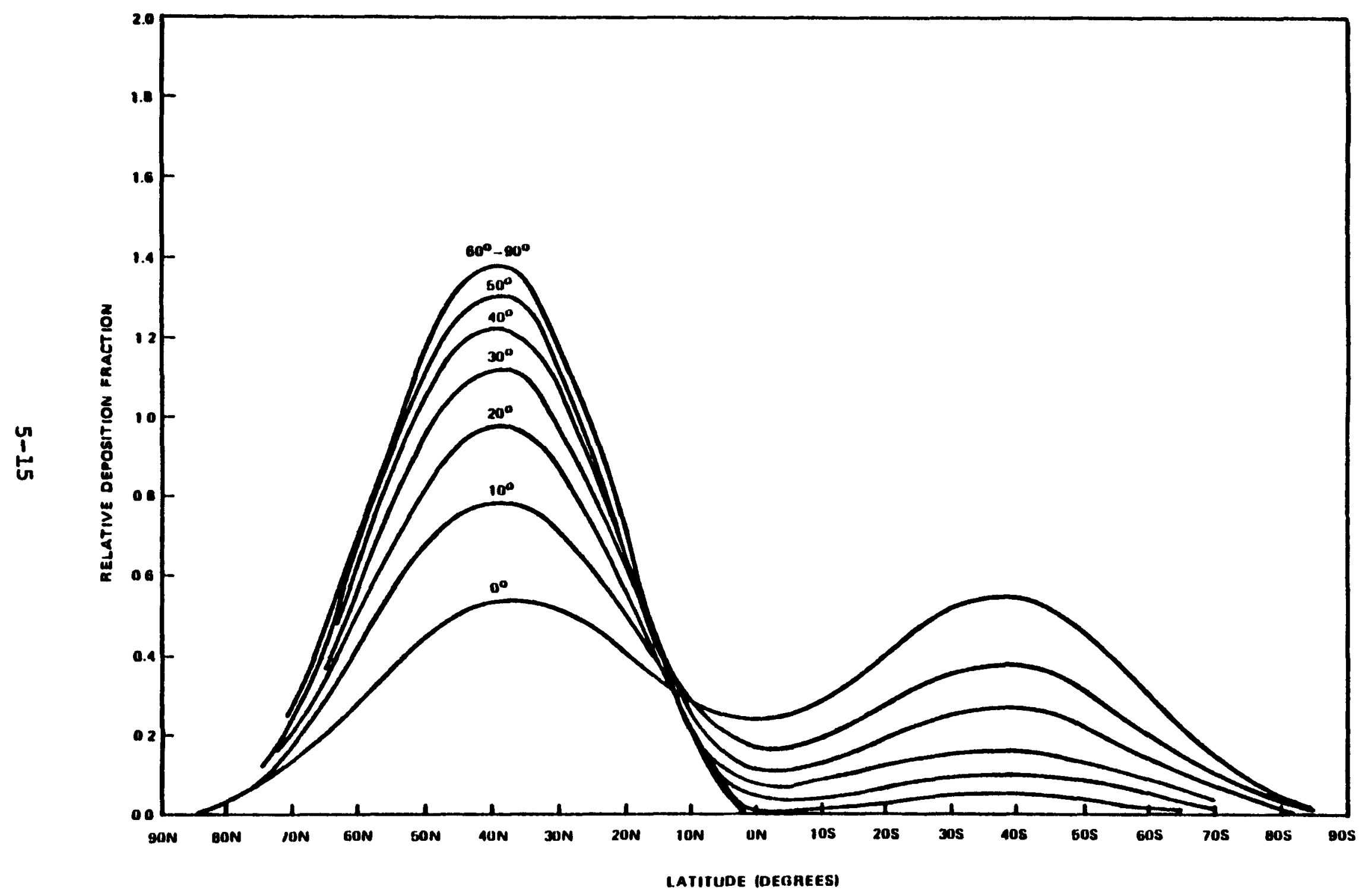

Figure 5-7. Deposition of small particles $(\leqslant 4 \mu)$ released at high altitude as a function of injection latitude. 
Table 5-3. Fraction of Source Falling in Each Equal Area Latitude Band as a Function of the Injection Latitude

\begin{tabular}{|c|c|c|c|c|c|c|c|c|c|c|c|}
\hline & & & & & Injer & Lun toat I & ude & & & & \\
\hline & $\begin{array}{l}\text { Buwal Area } \\
\text { ledIIlude Band }\end{array}$ & $02.50 \mathrm{y}$ & $200^{9}$ & & $200 \mathrm{~N}$ & $60^{\circ} \mathrm{y}$ & $-509 \mathrm{~N}$ & $400 \mathrm{~W}$ & 309 & $200 \mathrm{MI}$ & $10^{\circ}$ \\
\hline & 1 & .0727 & .0727 & & .0725 & .0210 & .0700 & .0672 & .0636 & .0560 & 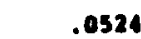 \\
\hline & 2 & .1470 & .1477 & & .1473 & .1450 & .1390 & .1317 & .1200 & .1064 & .0676 \\
\hline & 3 & .1707 & .1707 & & .1792 & .1753 & .1607 & .1504 & .1445 & .1263 & .1024 \\
\hline & 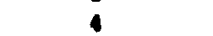 & .1745 & .1745 & & .1741 & .1712 & .1640 & .1548 & .1413 & .1236 & .1004 \\
\hline & 5 & .1606 & .1606 & & .1602 & .1576 & .1510 & .1120 & .1305 & .1145 & .0935 \\
\hline & 6 & .1109 & .1109 & & .1207 & .1091 & .1055 & .1001 & .0927 & .0030 & .07 \\
\hline & $i$ & .0823 & .0023 & & .0022 & .0012 & .0709 & .0754 & .0707 & .0644 & .0563 \\
\hline & 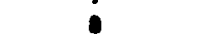 & .0474 & .0474 & & .0474 & .0471 & .0464 & .0454 & .0439 & .0421 & .0397 \\
\hline & 9 & .0170 & .0178 & & .0179 & .0182 & .0100 & .0199 & .0213 & .0231 & .0255 \\
\hline & 10 & .0074 & .0074 & & .0075 & .0000 & .0091 & .0100 & .0133 & .0164 & .0205 \\
\hline & $\begin{array}{l}11 \\
12\end{array}$ & 0.0000 & $\begin{array}{l}0.0000 \\
0.0000\end{array}$ & & $\begin{array}{r}.0001 \\
0.0000\end{array}$ & .0000 & $\begin{array}{l}.0024 \\
.0022\end{array}$ & $\begin{array}{r}.0049 \\
.0060\end{array}$ & .0002 & .0126 & .0183 \\
\hline & 13 & 0.0000 & 0.0000 & & .0002 & .0011 & .0030 & .0060 & .0115 & .0176 & .020 \\
\hline & 14 & 0.0000 & 0.0000 & & .0002 & .0014 & .0042 & .0005 & .0143 & .0219 & .0319 \\
\hline & 15 & 0.0000 & 0.0000 & & .0002 & .0017 & .0049 & .0100 & .0160 & .0257 & .03 \\
\hline & 16 & 0.0000 & 0.0000 & & .0003 & .0021 & .0060 & .0122 & .0205 & .0315 & .04 \\
\hline & 17 & 0.0000 & 0.0000 & & .0003 & .0022 & .0064 & .0129 & .0210 & .0333 & .0 \\
\hline & 10 & 0.0000 & 0.0000 & & .0003 & .0022 & .0065 & .0131 & .0221 & .0339 & .019 \\
\hline & 19 & 0.0000 & 0.0000 & & .0003 & .0019 & .0058 & .0116 & .0196 & .0300 & .0437 \\
\hline & 20 & 0.0000 & 0.0000 & & 0.0000 & .0013 & .0041 & .0002 & .0130 & .0211 & \\
\hline & & & & & Injectio & latitud & Band & & & & \\
\hline & $\begin{array}{l}\text { Kqual Area } \\
\text { Lat tytude Hand }\end{array}$ & $0^{\circ}$ & $100 \mathrm{~g}$ & $20 \%$ & 3008 & $109_{8}$ & 3008 & $60^{\circ}$ & $70^{\circ}$ & $00^{\circ} \mathrm{g}$ & 07.508 \\
\hline & $\mathbf{I}$ & .0437 & .0107 & .0211 & .0136 & .0082 & .0041 & .0013 & 0.0000 & 0.0000 & 0.0000 \\
\hline & 2 & .0622 & .0437 & .0300 & .0196 & .0116 & .0058 & .0019 & .0003 & 0.0000 & 0.0000 \\
\hline & 3 & .0703 & .0494 & .0339 & .0221 & .0131 & .0065 & .0022 & .0003 & 0.0000 & 0.0000 \\
\hline & 4 & .0691 & .0446 & .0333 & .0210 & .0129 & .0064 & .0022 & .0001 & 0.0000 & 0.0000 \\
\hline & 5 & .0652 & .0450 & .0115 & .0205 & .0122 & .0060 & .0021 & .0001 & 0.0000 & 0.0000 \\
\hline & 6 & .0532 & .0374 & .0257 & .0160 & .0100 & .0049 & .0017 & .0002 & 0.0400 & 0.0000 \\
\hline & 7 & .0453 & .0319 & .0219 & .0141 & .0085 & .0042 & .0011 & .0002 & 0.0000 & 0.0000 \\
\hline & - & .0363 & .0256 & .0176 & .0115 & .0068 & .0014 & .0011 & .0002 & 0.0000 & 0.0000 \\
\hline & 9 & .0208 & .0202 & .0139 & .0091 & .0060 & .0027 & .0009 & 0.0000 & 0.0000 & 0.0000 \\
\hline & 10 & .0260 & .0183 & .0126 & .0042 & .0049 & .0024 & .0000 & .0001 & 0.0000 & 0.0000 \\
\hline & 11 & .0260 & .0205 & .0164 & .0133 & .0100 & .0091 & .0000 & .0075 & .0024 & .0074 \\
\hline & 12 & .0280 & .0255 & .0231 & .0213 & .0199 & .0108 & .0142 & .0179 & .0170 & .0170 \\
\hline & 13 & .0363 & .0397 & .0421 & .0439 & .0454 & .0464 & .0471 & .0174 & .0474 & .0474 \\
\hline & 11 & .0453 & .0563 & .0644 & .0707 & .0754 & .0789 & .0012 & .0822 & .0823 & .0823 \\
\hline & 15 & .0532 & .0701 & (1) 30 & .0927 & .1001 & .1055 & .1091 & .1102 & .1109 & .1109 \\
\hline & 16 & .0652 & .0935 & .1145 & .1305 & .1420 & .1510 & .1576 & .1602 & .1606 & .1606 \\
\hline & 17 & .0691 & .1004 & .1236 & .1413 & .1518 & .1640 & .1712 & .1741 & .1745 & .1745 \\
\hline & iu & .0701 & .1024 & .1263 & .1445 & .1584 & .3607 & .1753 & .1782 & .1787 & .1787 \\
\hline & 19 & .0622 & .0476 & .1064 & .1206 & .1311 & .1390 & .1450 & .1173 & .1477 & .1478 \\
\hline & 20 & .0437 & .0524 & .0588 & .0636 & .0672 & .0700 & .0718 & .0725 & .0727 & .0727 \\
\hline
\end{tabular}




$$
s_{i j}=\sum_{k=1}^{20} s_{j k} z_{i k}
$$

where

$F_{i j}=$ fraction of high altitude vapor released at injection latitude $j$ transferred to surface type $i$

$F_{i k}=$ fraction of high altitude vapor released at injection latitude $j$ transferred to equal area latitude band $k$

$P_{i k}=$ fraction of surface type $i$ in equal area latitude band

Accounting for the latitudinal distribution of vapor released at high altitude, the ground level normalized concentration in each equal area latitude band is

$$
\begin{aligned}
\frac{x_{j k}(0, t)}{Q(0)} & =\frac{20 f_{j k} Q_{T}(t) a}{M_{T} Q(0)} \\
& =20 f_{j k} \frac{\bar{x}(0, t)}{Q(0)}
\end{aligned}
$$

where

$$
\begin{aligned}
& j k(0, t)= \text { ground level concentration at time } t \text { in equal area } \\
& \text { latitude band } k \text { due to a high altitude band } k \text { due } \\
& \text { to a high altitude vapor release in injection lati- } \\
& \text { tude band } j \text { at time } t=0,9 / \mathrm{m}^{3}
\end{aligned}
$$

The normalized population exposure is

$$
\begin{aligned}
\frac{E(t)}{Q(0)} & =\frac{1}{Q(0)} \sum_{k=1}^{20} x_{j k}(0, t) p_{k} \\
& =\frac{20 \bar{x}(0, t)}{Q_{(0)}} \sum_{k=1}^{20} s_{j k} z_{k} \\
& =\frac{\bar{x}(0, t) R_{j}}{Q(0)} P_{T}
\end{aligned}
$$


where

Pk = total population in equal area latitude band $k$

Rj = population risk factor for high altitude vapor releases in injection latitude $j$, persons

$$
-20 \sum^{20} \frac{E_{j k}{ }^{2} k}{P_{T}}
$$

$k=1$

$E(t)=$ population exposure at $t$ ime $t$, person-g/m $\mathrm{m}^{3}$

$\mathbf{P}_{\mathbf{T}}=$ total world population $\left(3.79 \times 10^{9}\right.$ persons $)$

A plot of the population risk factor $R_{j}$ as a function of injection latitude is present in Figure 5-8.

Op to this point only the instantaneous ground level mass concentration of vapor released at high altitude as a function of time has been addressed. The radiological doses to the general population due to vapor released at high altitude will be a function of the time integrated ground concentration, accounting properly for radioactive decay.

The instantaneous ground level concentrations calculated previously were in terms of $\mathrm{g} / \mathrm{m}^{3}$. To convert to radioactivity concentrations, note that

$$
\begin{aligned}
x(0, t)\left[\frac{c_{i}}{\mathrm{~m}^{3}}\right] & =x(0, t)\left[\frac{g}{\mathrm{~m}^{3}}\right]\left(\frac{\mathrm{A}_{0}}{\mathrm{M}_{0}}\right)\left(\frac{1 \mathrm{Ci}}{3.7 \times 10^{10 \mathrm{dis} / \mathrm{sec}}}\right) \\
& x\left[\frac{1 \mathrm{y}=}{(365 \text { days })(24 \mathrm{hr} / \mathrm{day})(3600 \mathrm{sec} / \mathrm{hI})}\right]
\end{aligned}
$$

where

$$
\begin{aligned}
A_{0} & =\text { Avogodro's number } \\
& =6.023 \times 10^{23} \text { atoms/gram-mole } \\
M_{0} & =\text { gram-molar weight } \\
\lambda & =\text { radioactive decay constant, } y^{-1}
\end{aligned}
$$

For fission products and transuranics released at high altitude, the calculation of the time integrated ground level concentration is not straight forward. Because of the large number of radionuclides of interest, many of which having more than one radioactive daughter that buildup, the time integration was performed numerically in discrete time steps. The time integrated ground level concentration for a given radionuclide was approximated by 


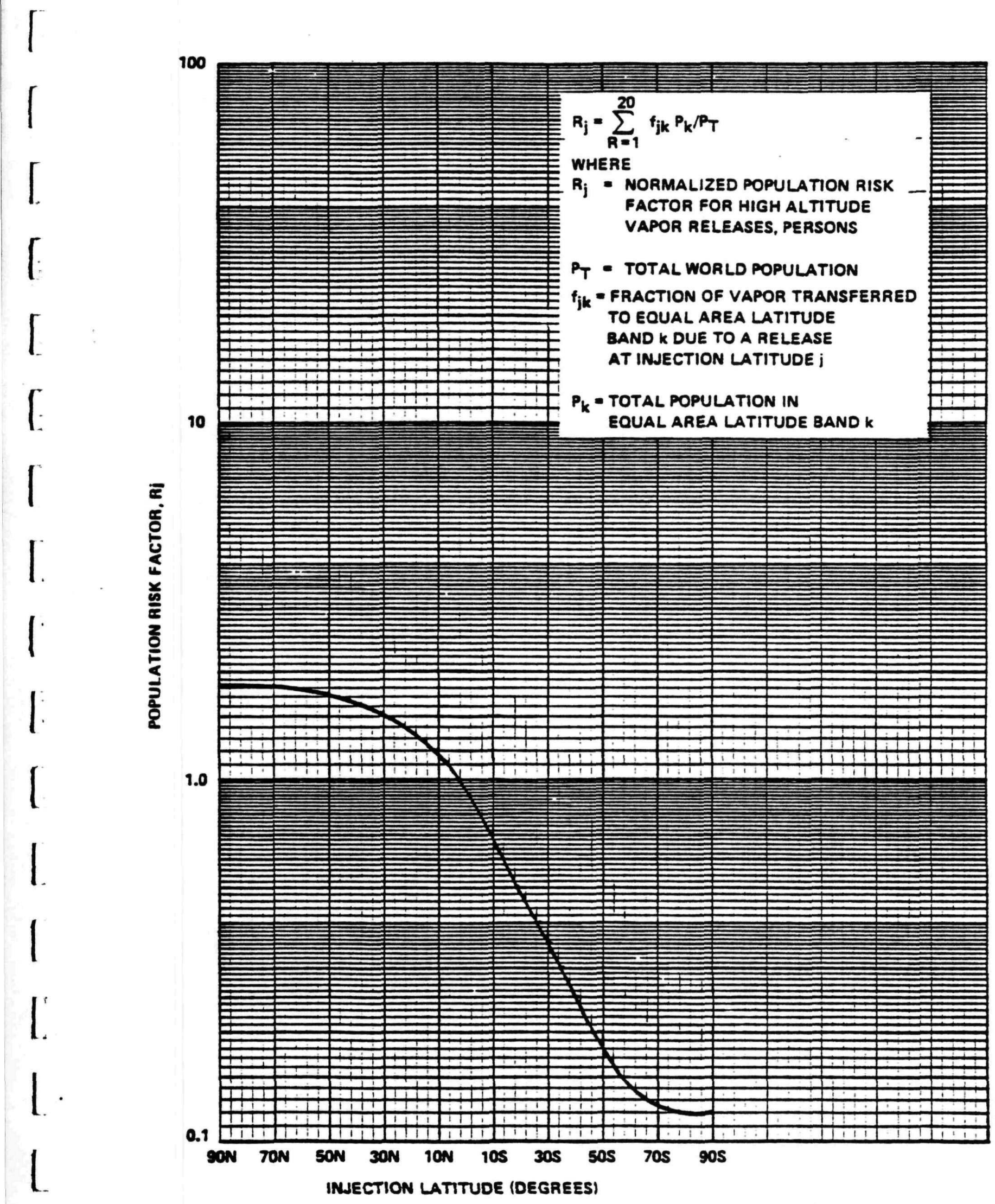

Figure 5-8. Normalized population risk factor as a function of injection latitude for high altitude vapor releases.

$$
\text { 5-19 }
$$




$$
\begin{aligned}
\psi_{i} & =\int_{0}^{\infty} \frac{x(0, t)}{Q(0)} q_{i}(t) d t \\
& =\sum_{j=1}^{n} g_{i}\left(t_{j}\right) \int_{t_{j-i}}^{t_{j+1}} \frac{x\left(0, t^{-}\right) d t^{-}}{Q(0)}
\end{aligned}
$$

where

$$
\begin{aligned}
& \psi_{i}= \begin{array}{l}
\text { time integrated ground level concentration of } \\
\text { radionuclide } i,
\end{array} \\
& q_{i}\left(t_{j}\right)= \begin{array}{r}
\text { total inventory of radionuclide } i \text { at time } t_{j}, \\
\text { curie }
\end{array} \\
& \frac{x(0, t)=}{(0)}=\begin{array}{l}
\text { ground level normalized concentration at time } \\
t \text { without radioactive decay, } y r / \mathrm{m}^{3}
\end{array}
\end{aligned}
$$

The ORIGIN computer program developed by Oak Ridge National Laboratory was used to calculate the inventory of fission products and transuranics as a function of time after shutdown. (18)

The integrated form of the integral between times $t_{j-I}$, and $t_{j+1}$ for mesosphere releases, using Equation $(5-4), j \bar{i}^{-1}$

$$
\begin{aligned}
\int_{t_{j-1}}^{t_{j+1}} \frac{x\left(0, t^{\prime}\right)}{a_{M}(0)} d t^{-}= & \frac{\lambda_{1} \lambda_{2}}{M_{1}}\left[\frac{e^{-\lambda_{1} t_{j-1}}-e^{-\lambda_{1} t_{j+1}}}{\lambda_{1}\left(\lambda_{3}-\lambda_{1}\right)\left(\lambda_{2}-\lambda_{1}\right)}+\frac{e^{-\lambda_{2} t_{j-1}}-e^{-\lambda_{2} t_{j-1}}}{\lambda_{2}\left(\lambda_{3}-\lambda_{2}\right)\left(\lambda_{1}-\lambda_{2}\right)}\right. \\
& \left.+\frac{e^{-\lambda_{3} t_{j}-1}-e^{-\lambda_{3} t_{j+1}}}{\lambda_{3}\left(\lambda_{1}-\lambda_{3}\right)\left(\lambda_{2}-\lambda_{3}\right)}\right]
\end{aligned}
$$

The integrated form of the integral between times $t_{j-1}$ and $t_{j+1}$
for stratosphere releases, using Equation $(5-8)$ is

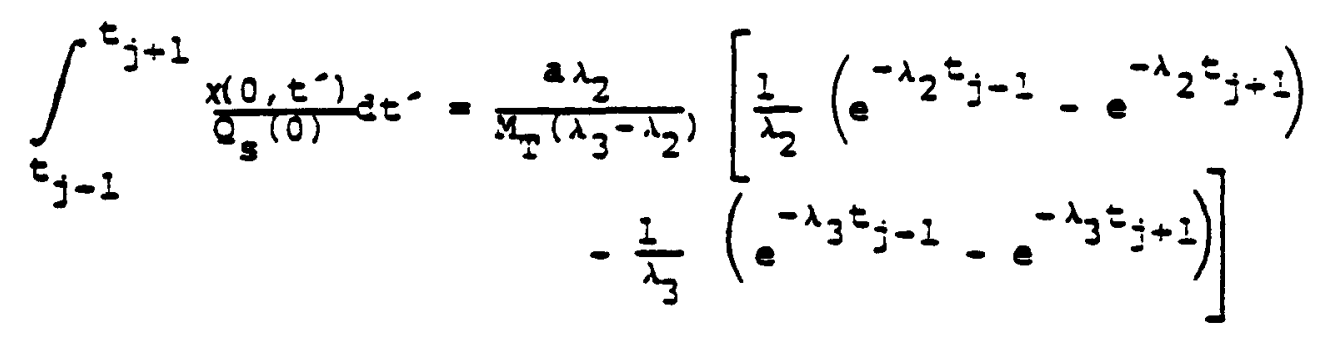


The integrated form of the integral between times $t_{j-1}$ and $t_{j+1}$
for troposphere releases, using Equation $(5-10)$ is

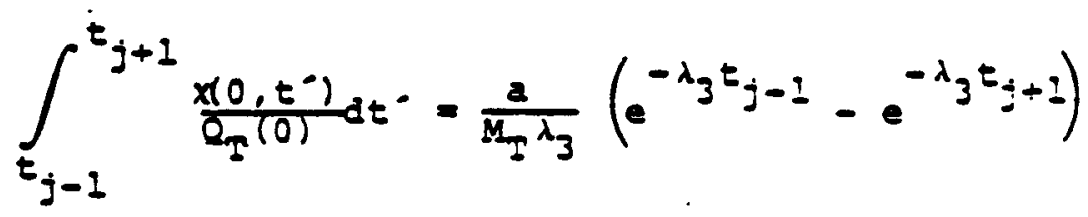

The normalized time integrated ground level concentrations for discrete time periods corresponding to those used in the ORIGIN source term calculation were evaluated and the results are summarized in Figure 5-9.

Resuspended air concentrations are not calculated for high altitude vapor releases because removal rates from each atmospheric compartment are based on ground level measurements as a function of time that already account for resuspension.

In addition to the time integrated ground level airborne concentrations during discrete time periods, other information of a similar nature is required for the radiological dose calculations. This information include the ground plane and vegetation concentrations as a function of time due to deposition.

The ground plane concentration as a function of time due to high altitude vapor releases is

$$
\omega(t)=\int_{0}^{t} D\left(t^{-}\right) e^{-\lambda_{w}\left(t-t^{\prime}\right)} d t^{\prime}
$$

where

$$
\begin{aligned}
\omega(t) & =\text { ground plane concentration at } t j m e t, g / m^{3} \\
D\left(t^{\prime}\right) & =\text { deposition rate at time } t, g / \mathrm{m}^{2}-y r \\
\lambda_{w} & =\text { ground plane weathering removal rate, }
\end{aligned}
$$




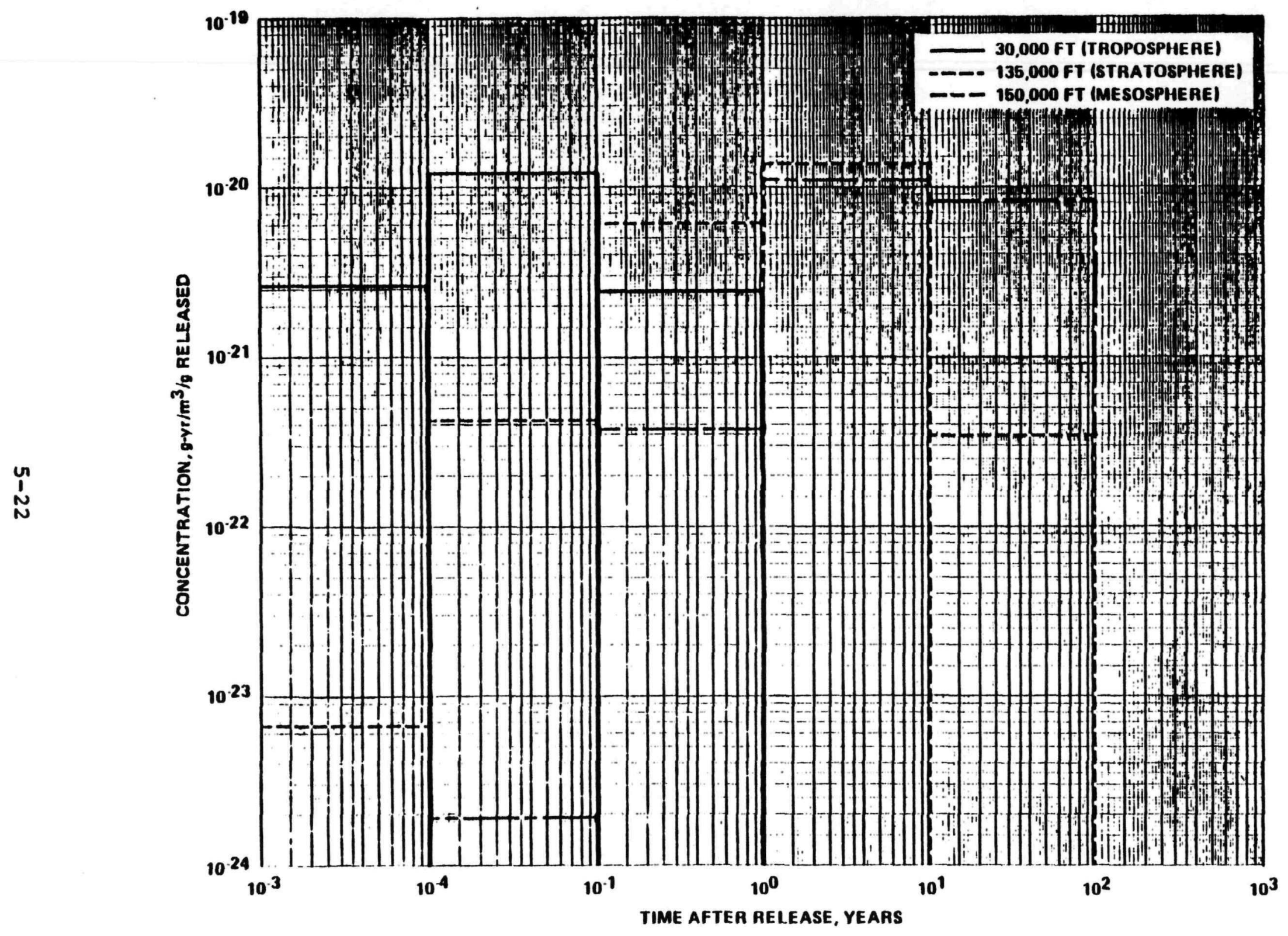

Figure 5-9. Time integrated ground level airborne mass concentration for discrete time periods due to high altitude vapor releases. 
The ground level deposition rate due to vapor releases in the mesosphere is determined from the time derivative of Equation $(5-5)$. Hence,

$$
\begin{aligned}
& D(t)=\frac{1}{\lambda} \frac{d Q_{g}(t)}{d E} \\
& =-\frac{Q_{1}(0) \lambda_{1} \lambda_{2} \lambda_{3}}{\lambda}\left[\frac{e^{-\lambda_{1} t}}{\left(\lambda_{1}-\lambda_{2}\right)\left(\lambda_{3}-\lambda_{1}\right)}+\frac{e^{-\lambda_{2} t}}{\left(\lambda_{2}-\lambda_{1}\right)\left(\lambda_{3}-\lambda_{2}\right)}-\frac{e^{-\lambda_{3}}=}{\left(\lambda_{3}-\lambda_{1}\right)\left(\lambda_{2}-\lambda_{3}\right)}\right]
\end{aligned}
$$

where

$$
\begin{aligned}
A & =\text { earth's surface area, } \mathrm{m}^{2} \\
& =5.168 \times 1013 \mathrm{~m}^{2}
\end{aligned}
$$

Integrating Equation (5-24) using Equation (5-25) then the worldwide average ground plane concentration is

$$
\begin{aligned}
I(t)=\frac{\rho_{m}(0) \lambda_{1} \lambda_{2} \lambda_{3}}{\lambda} & {\left[\frac{e^{-\lambda_{1} t}-e^{-\lambda_{w t}}}{\left(\lambda_{1}-\lambda_{w}\right)\left(\lambda_{1}-\lambda_{2}\right)\left(\lambda_{3}-\lambda_{1}\right)}+\frac{e^{-\lambda_{w} t}-e^{-\lambda_{w t}}}{\left(\lambda_{2}-\lambda_{w}\right)\left(\lambda_{2}-\lambda_{1}\right)\left(\lambda_{3}-\lambda_{2}\right)}\right.} \\
& \left.+\frac{e^{-\lambda_{3} t}-e^{-\lambda_{w t}}}{\left(\lambda_{3}-\lambda_{w}\right)\left(\lambda_{3}-\lambda_{1}\right)\left(\lambda_{2}-\lambda_{3}\right)}\right]
\end{aligned}
$$

Following a similar procedure the ground level concentrations due to stratosphere vapor releases using Equation (5-9) is

$$
\bar{w}(t)=\frac{Q_{5}(0) \lambda_{2} \lambda_{3}}{A\left(\lambda_{2}-\lambda_{3}\right)}\left[\frac{e^{-\lambda_{2} t}-e^{-\lambda_{w} t}}{\left(\lambda_{2}-\lambda_{w}\right)}-\frac{e^{-\lambda_{3} t}-e^{-\lambda_{w} t}}{\left(\lambda_{3}-\lambda_{w}\right)}\right]
$$

The ground level concentrations due to troposphere vapor releases using Equation $(5-11)$ is

$$
\bar{H}(t)=\frac{Q_{I}(0) \lambda_{3}}{A\left(\lambda_{3}-\lambda_{w}\right)}\left[e^{-\lambda_{w} t}-e^{-\lambda_{3} t}\right]
$$

The normalized ground concentration as a function of time for various release altitudes is shown in Figure 5-10. 
To account for the latitudinal distribution of ground concentration resulting from each injection latitude, note that

$$
\frac{\omega_{j k}(t)}{Q(0)}=20 \varepsilon_{j k} \frac{\bar{\omega}(t)}{Q(0)}
$$

where

$$
\begin{aligned}
\omega_{j k}(t)= & \text { ground plane concentration at time } t \text { in equal area } \\
& \text { latituge band } k \text { due to a release in injection latitude } \\
& j, g / m
\end{aligned}
$$

fjk has been defined previously in Equation (5-17) and given in Table 5-3.

The concentration of fallout vapor released at high altitude on foliar vegetation due to deposition is

$$
C_{\nabla}(t)=\int_{0}^{t} D(t) e^{-\lambda_{v}\left(t-t^{0}\right)} d t^{-}
$$

where

$$
\begin{aligned}
C_{v}(t)= & \text { vegetation concentration at } t \text { ime } t, g / g \text { of vegetation } \\
r= & \text { concentration factor, } g / \mathrm{m}^{2} \\
\lambda_{v}= & \text { removal rafe from vegetation, } \mathrm{yr}^{-1} \\
= & 18.068 \text { yr } \mathrm{I} \text { (based on a } 14 \text { day removal half life } \\
& \text { based on Reference 22) }
\end{aligned}
$$

Equation (5-29) for vegetation concentration is completely analogous to Equation (5-24) for ground plane concentrations. Therefore, Equations 5-26, 5-27, and 5-28 for ground plane concentrations due to vapor releases in the mesosphere, stratosphere, and troposphere may be used to calculate the corresponding vegetation concentration, $C_{v}(t)$, with the change $\lambda_{w} \rightarrow \lambda_{v}$ and $Q(0) \rightarrow r Q(0)$. The resulting normalized vegetation concentration as a function of time for various release altitudes is shown in Figure 5-11. The latitudinal dependence of vegetation concentration will be the same as that for the ground plane concentrations, as expressed by Equation $(5-29)$.

\subsubsection{Eigh Altitude Particulate Releases}

A high altitude particulate release $(\geq 4 \mu \mathrm{m}$ in physical diameter) can result from the disassembly of all or part of an alternative power system as a result of planned or unplanned destruction scenarios. The resulting particulates will follow trajectories to the earth's surface that are determined by the mass, size, and 
shape of each particle, the release altitude, as well as the wind and density profile of the atmosphere.

An evaluation of time of flight from a given release altitude to ground level has been performed based on terminal fall velocities. This approach is valid assuming that vertical wind speeds from the release altitude to ground level average out to be zero. The terminal fall velocity of a given particle can be calculated according to its aerodynamic regime as summarized in Table 5-5.

The terminal fall velocity for particles in the stokes Region, representing those particles of primary interest is

$$
v_{s}=\frac{g D^{2}(p-P a)}{1.8 \times 10^{9_{\mu}}}
$$

where

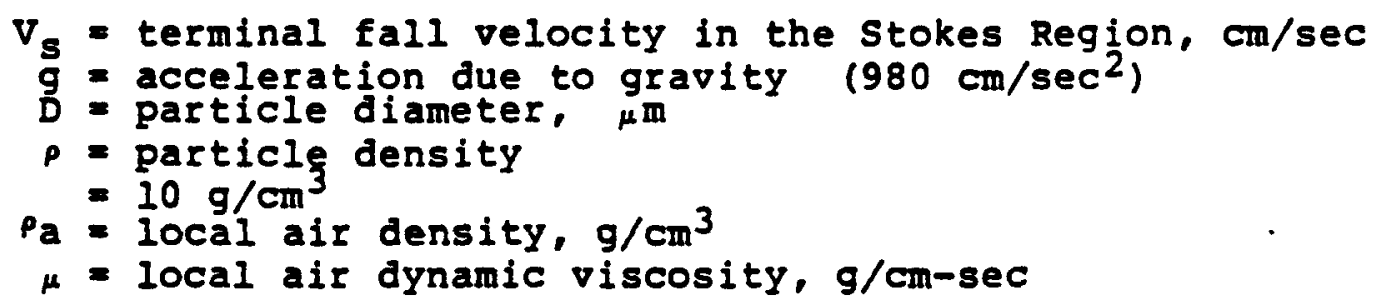

In the altitude range of $0-45 \mathrm{~km}$, the air density, $\rho_{a}$, ranges from $1.2 \times 10^{-3}$ to $1.8 \times 10^{-6} \mathrm{~g} / \mathrm{cm}^{3}$ which can be neglected compared to the particle density, $p$, of $10 \mathrm{~g} / \mathrm{cm}^{3}$. The dynamic viscosity of air has a corresponding range of $1.79 \times 10^{-4}$ to 1.67 $\times 10^{-4} \mathrm{~g} / \mathrm{cm}-\mathrm{sec}$ with a minimum of $1.42 \times 10^{-4} \mathrm{~g} / \mathrm{cm}-\mathrm{sec}$ between 11 to $20 \mathrm{~km}$. The ayerage dynamic viscosity through this altitude range is $1.53 \times 10^{-4} \mathrm{~g} / \mathrm{cm}-\mathrm{sec}$. This order of magnitude of the dynamic viscosity holds throughout the atmosphere, and the average value cited is considered representative for any high altitude point of release. $(26)$ The time of flight, $t$, in years from a given release altitude, $B$, in kilometers is

$$
t=\left(\frac{\mathrm{E}}{V_{s}}\right)\left(\frac{1000 \mathrm{~m}}{\mathrm{~g}}\right)\left(\frac{100 \mathrm{gm}}{\mathrm{ag}}\right)\left(\frac{1 \mathrm{hI}}{3600 \mathrm{sec}}\right)\left(\frac{1 \mathrm{dav}}{24 \mathrm{hI}}\right)\left(\frac{1 \mathrm{vI}}{365 \mathrm{days}}\right)
$$

Substituting for $B$ and $V_{s}$ using the appropriate input parameter values identified above, then

$$
t=\frac{8.91 \times 10^{-2} \mathrm{H}}{D^{2}}
$$


Table 5-5. Aerodymantc Regimes for Given Particle size Ranges.

Aerodynamic

Diameter Range,

Regime

$\operatorname{um}^{\mathrm{a}}$

Diameter Range for

Stokes-Cunningham

stokes

Intermediate

Newtons

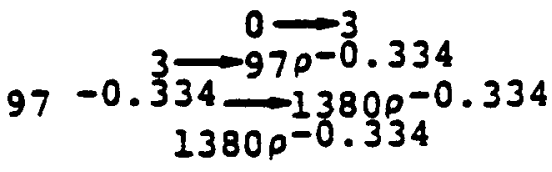

Fuel,amb

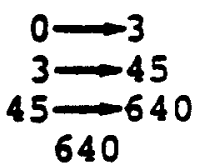

a $p=$ particle density, g/ $\mathrm{cm}^{3}$.

bThe density of fuel is assumed to be $10 \mathrm{~g} / \mathrm{cm}^{3}$. 
The particle descent time as a function of release altitude and particle size, based on Equation (5-37), is shown in Figure 5-14. A comparison of the descent times shown in Figure 5-12 and the ground level airborne concentration due to high altitude vapor releases shown in Figure 5-7 indicates the vapor model is representative of particle sizes greater than $4 \mu \mathrm{m}$, up to $20 \mu \mathrm{m}$ depending on the release altitude. At this point a critical assumption will be made related to this information. It is assumed the high altitude vapor model completely characterizes all particle in the inhalable range. A model will be described further below for fullout particulates that deposit on the ground but are not inhaled.

The time delay associated with particulates released at high altitudes in the non-inhalable range is assumed to be characterized by that for $45 \mu \mathrm{m}$ particles shown in Figure 5-12. The fallout particulates are assumed to be restricted to the latitude band within which breakup occurs, with random earth surface characteristics with the band presented in Table 5-2.

The atmospheric dispersion of such particulates released at high altitudes has been treated in Reference 1 using a modified version of the Model $\left(\mathrm{B}_{1}\right.$ fomputer code developed by the Travelers Research Corporation. $(21)$

In the Model B code, the trajectories of individual mass elements are obtained by numerical integration of ordinary differential equations for the rates of change of colatitude, longitude, and altitude, using local wind speed components and the terminal fall velocity of the particles.

Using this model, parametrics were generated as a function of release height at a $45^{\circ}$ latitude using an assumed particle size distribution presented in Figure 5-13.(2) typical of fractured $\mathrm{PuO}_{2}$ fuel and assumed to be representative in the present case. The resultant initial normalized ground deposition density versus affected area for each release altitude is presented in Figure 5-14. (2)

The parametrics for other latitudes are similar. The mass concentration on the ground after release is

$$
\omega(t)=\omega(0) e^{-\lambda \omega t}
$$

where $\lambda_{w}$ and $w$ have been defined previously in Equation 5-29. The corresponding concentration on vegetation 'is

$$
c_{v}(t)=\omega(0) e^{-\lambda} t
$$




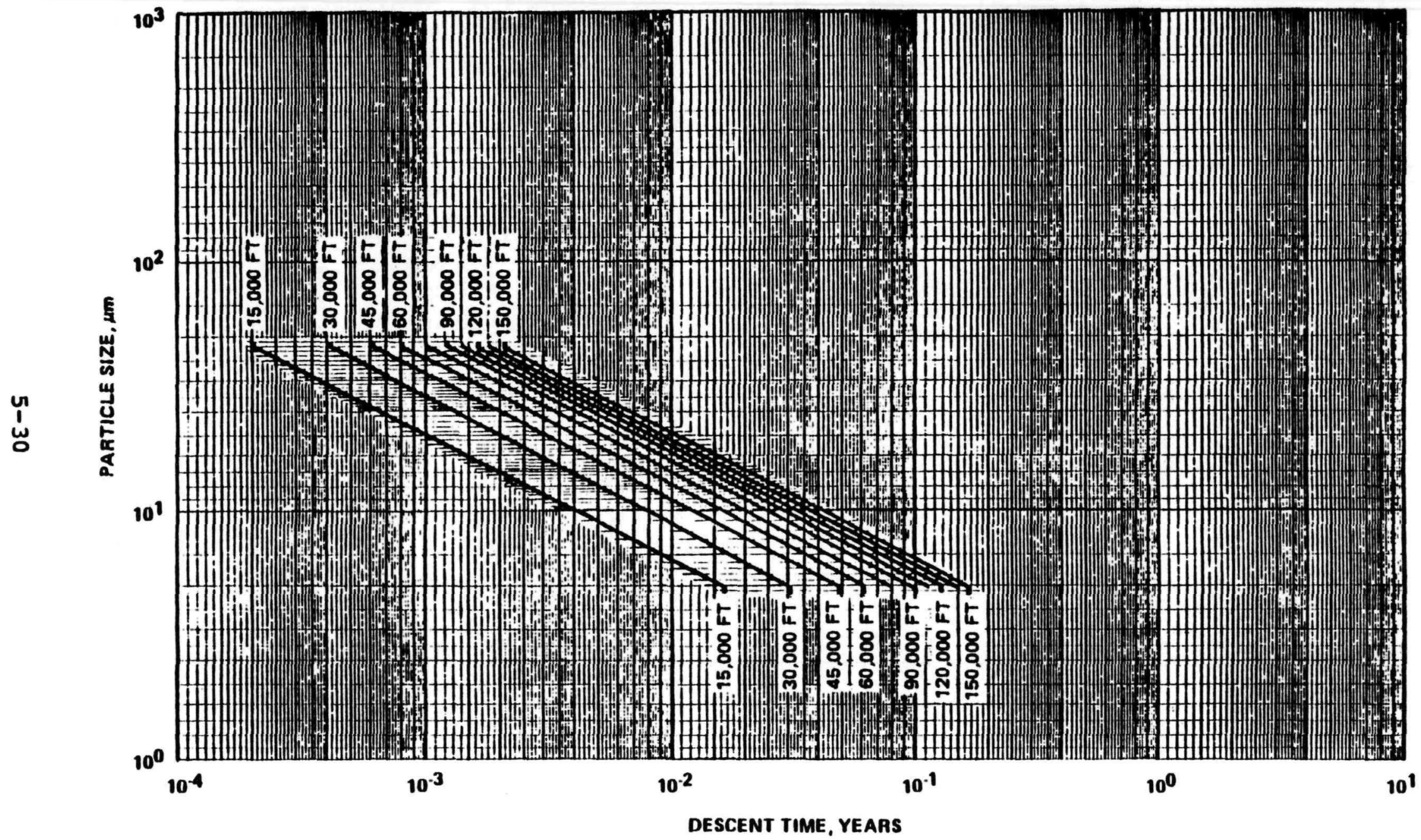

Figure 5-12. Descent times for particulates released at high altitudes as a function of release height and particle size. 


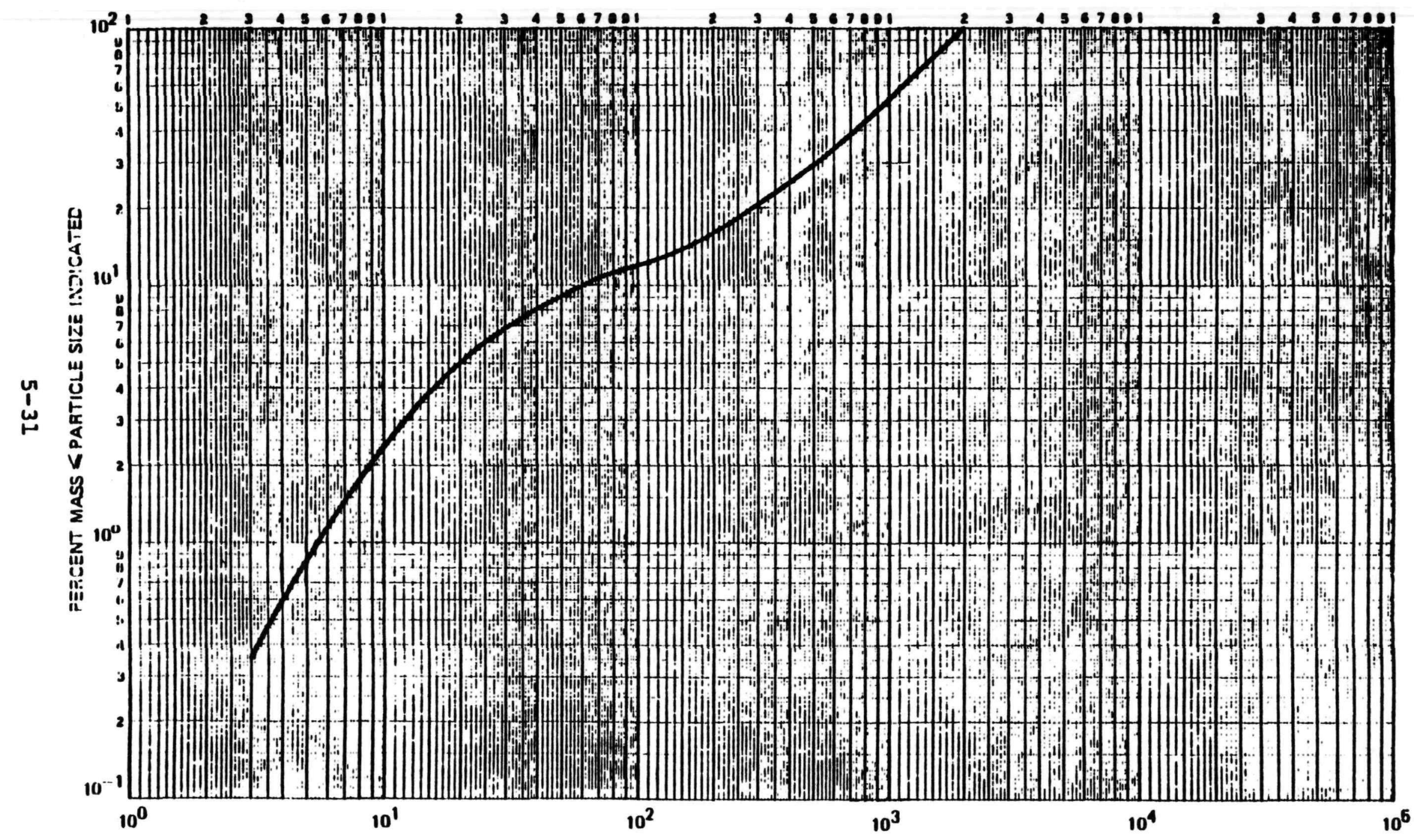

Figure 5-13. Cumulative mass distribution for particles less than or equal to 2000 microns. 


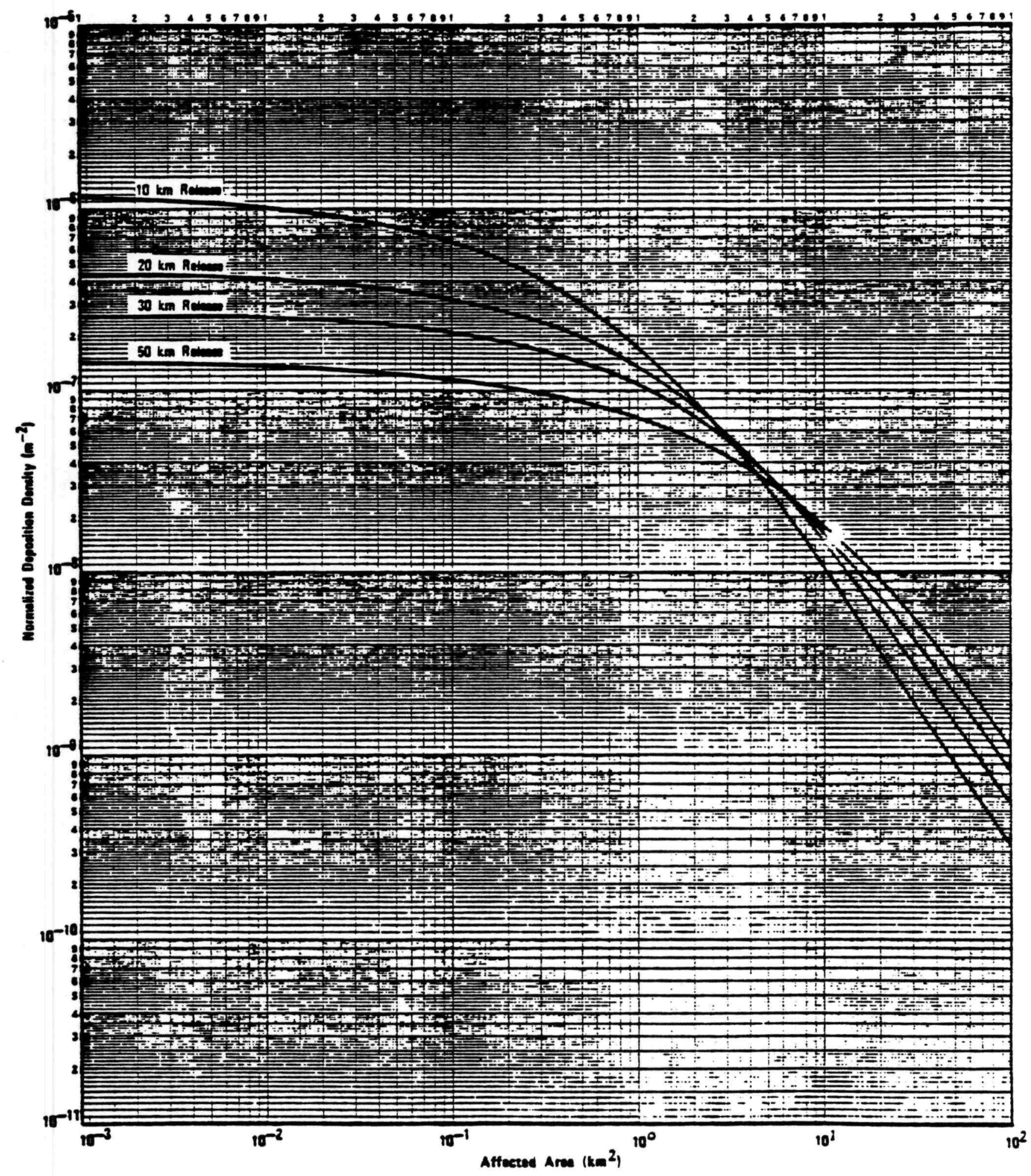

Figure 5-14. Normalized deposition density $\left(\mathrm{m}^{-2}\right)$ as a function of affected area $\left(\mathrm{km}^{2}\right)$ for various release heights at $45^{\circ}$ latitude. 
where $C_{v}(t), r$, and $\lambda_{v}$ have been defined previously in Equation 5-30.

Figure 5-14 and Equations 5-33 and 5-34 provide inputs to the radiological dose models described in Chapter 6.

Weathering of the ground plane deposit will result in the conversion of larger particulates into smaller particulates that become subject to resuspension.

The resuspended airborne concentration can be written as

$$
\begin{aligned}
x_{R}(t) & =R(t) \omega(t) \\
& =R(t) \omega(0) e^{-\lambda_{w} t}
\end{aligned}
$$

where

$$
\begin{aligned}
x_{R}(t) & =\text { suspended airborne concentration at } t \text { ime } t, g / m^{3} \\
R & =\text { resuspension factor at time } t, m^{-1} \\
\bar{\omega}(t) & =\text { average ground concentration at } t i m e t, g / m^{2}
\end{aligned}
$$

Experimental studies of the resuspension of plutonium dioxide par$t$ iculates indicated that the resuspension factor, $R(t)$ was $10^{-5}$ initially, decreasing exponentially to $10^{-9}$ after two years. After 2 years the fesuspension factor decreased at a rate of 108 per year. $\{27,28\}$ The time behayior of the resuspension factor can therefore be written as (1)

$$
R(t)=\begin{aligned}
& 10-5 e^{-4.6052 t} \text { for } t 2 \text { years } \\
& 10-9 e^{-0.1052 t} \text { for } t 2 \text { years }
\end{aligned}
$$

The average resuspended concentration over a given time step

$$
\begin{aligned}
\Delta t_{j} & =t_{j+1-t_{j-1} \text { is }} \\
\bar{x}_{R}\left(t_{j}\right)= & \frac{\omega(0)}{\Delta t_{j}}\left[\int_{0}^{2} 10^{-5} e^{-\left(4.605+\lambda_{w}\right) t} \theta\left(t-1 t_{j}\right) d t\right. \\
& \left.+\int_{2}^{\infty} 10^{-9} e^{-\left(0.105+\lambda_{w}\right) t} \theta\left(t-\Delta t_{j}\right) d t\right]
\end{aligned}
$$


where

$$
(t-2 t)=\left\{\begin{array}{lll}
1 & \text { if } t_{j-1} \leq t \leq t_{j+1} \\
0 & \text { if } t & t
\end{array}\right.
$$

The integrated airborne concentration during time $\Delta t$ due to resuspension is

$$
\psi_{R}\left(t_{j}\right)=\bar{x}_{R}\left(t_{j}\right) s t_{j}
$$

\subsubsection{LOW ALTITUDE RELEASE MODEL}

Low altitude dispersion of fuel can result from elevated releases associated with an explosion or fire, ground level releases of impacted fuel or resuspension of ground deposited particulates. Models will be presented for evaluation of maximum individual doses and population doses.

\subsubsection{Maximum Individual Exposures}

The airborne and ground mass concentrations associated with point source 5 fleases have been calculated with the XOQDOQ computer model $(29)$ used by the $0 . S$. Nuclear Regulatory Comission (NRC) to implempnt the calculations outlined in NRC Regulatory Guide 1.111. (30) The model calculates integrated direct airborne and ground mass concentrations at selected receptor locations using a straight line Gaussian model modified from Turnef (31) The model includes plume rise based on Briggs' formulation. $(32)$ Dry deposition is calculated based on depletion curves presented in Reference $(30)$ which are in turn based on an effective deposition velocity of $0.01 \mathrm{~m} / \mathrm{sec}$. The $X 0 Q D O Q$ model uses as inputs the annual meteorological joint-frequency distribution (JFD) of wind speed, wind direction, and atmospheric stability class, and the release conditions for each accident scenario considered, including the release height and fire heat flux (if any), and calculates the integrated direct airborne concentration, $x / Q$, and the normalized initial ground concentration, $w / Q$ at selected receptor locations.

The JFD used in the analysis was based on a worldwide meteorological study presented in References 2 and 33 . Composite JFDs for representative weather stations from 14 worldwide climatological categories were generated in Reference 33. The expected mean wind speed and atmospheric stability class for random impacts along great circle routes described by angle $\eta$ (see Section 5.1.1.2) are shown in Figures 5-15 and 5-16 based on the distribution of the 14 climatological categories. (2) For the present analysis, the meteorological JFD corresponding to a great circle inclination of $\eta=90^{\circ}$ was used, as presented in Table 5-6. The JFD essentially represents a worldwide average. The variability shown 


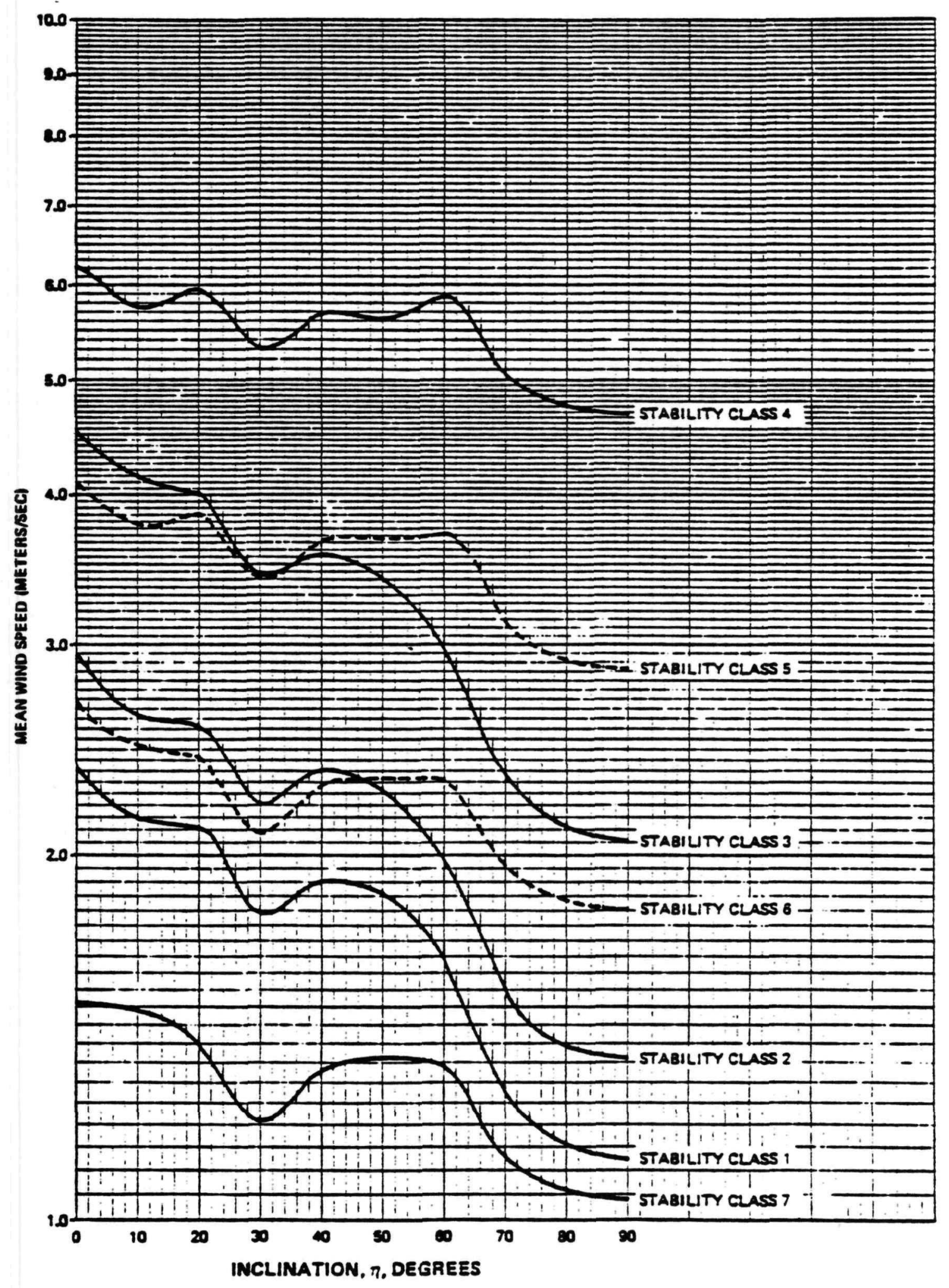

Figure 5-15. Expected wind speed for random impacts along great circle routes defined by $\eta$. 


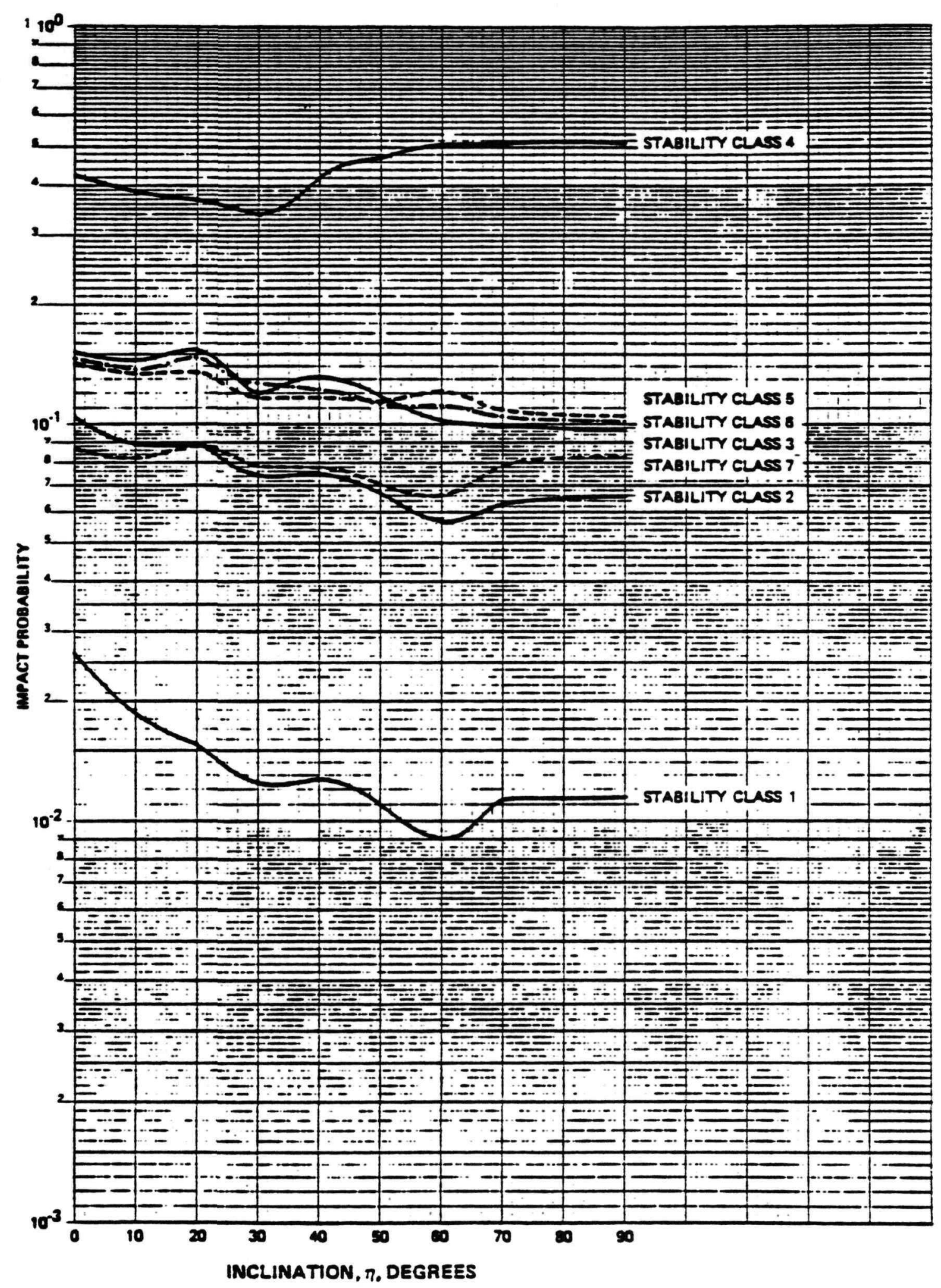

Figure 5-16. Atmospheric stability class for random impacts along great circle routes defined by $\eta$. 
Table 5-6. Worldwide Arerage Joint Frequency Distribution of wlnd Speed and Stability Class (Percent)

Midpoint

Hind Speed

\section{Stability Class}

\section{Inots}

m/sec

$\mathbf{A}$

B

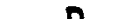

0

0

0.515

0.904

1.319

1.454

D

$\underline{E}$

E $\quad$ G

0.038

0.048

0.0

1.994

6.112

0.0290 .034

0.256

0.374

0.0

0.0

0.051

0.254

1.544

1.146

1.044

0.0

0.168

0.656

4.388

2.023

0.444

1.021

3.346

1.740

3.372

4.791

0.472

2.225

4.951

5.920

0.0

0.0

0.0

1.742

13.634

7.979

0.0

0.0

0.338

9.565

10.296

0.0

0.0

10.900

2.738

3.117

2.002

0.0

0.795

2.042

4.892

2.336

0.0

0.0

0.173

0.0

0.0

0.0

0.0

0.0

Total

$1.683 \quad 6.394$

9.784

52.145

$9.880 \quad 10.512$

9.602

I

1

1

1

1

1 
in Figures 5-15 and 5-16 relative to $\eta=90^{\circ}$ is not considered significant in the context of the error bands of the entire analysis. since the actual point of impact is hypothetical, there is no wind directionality associated with the analysis (i.e. all directions equally probable).

A series of case runs were made to calculate normalized integrated ground level airborne concentrations and ground concentrations as a function of distance. The cases consisted of unit source terms ranging from ground level release.

In addition to the direct air and ground concentrations calculated by $X O Q D O Q$, resuspended air concentrations during subsequent time periods were calculated using the resuspension model described by Equations 5-35 through 5-37 of section 5.1.1.2. Associated vegetation concentrations were calculated in accordance with Equation 5-30.

The results of the calculations are summarized in Figures 5-17 through 5-19 for the initial direct integrated ground level airborne concentration, ground concentration and vegetation concentrations as a function of downwind distance. The resulting concentrations were used in the maximum individual dose calculations as described in chapter 6 .

\subsubsection{General Population Exposures}

Exposure of the general population due to low altitude releases must account for vanishing small concentrations at great distances from the point of impact because such concentrations are important when the Iinear dose response model is used.

All the activity released and dispersed atmospherically will eventually be deposited on the ground. The total activity initially deposited is $(34)$

$$
Q(0)=\int_{0}^{2 \pi} \int_{0}^{R} \omega(E, \theta)=d z d \theta
$$

where

$$
\begin{aligned}
Q(0)= & \begin{array}{l}
\text { total fuel released through atmospheric } \\
\text { dispersion at time t=0, gram }
\end{array} \\
\omega(r, \theta)= & \text { total ground concentration after initial } \\
& \text { deposition at distance } r \text { in direction } \theta, g / \mathrm{m}^{2} \\
R= & \begin{array}{l}
\text { An arbitrary distance from the release point with- } \\
\text { in which the released activity is contained, m }
\end{array}
\end{aligned}
$$




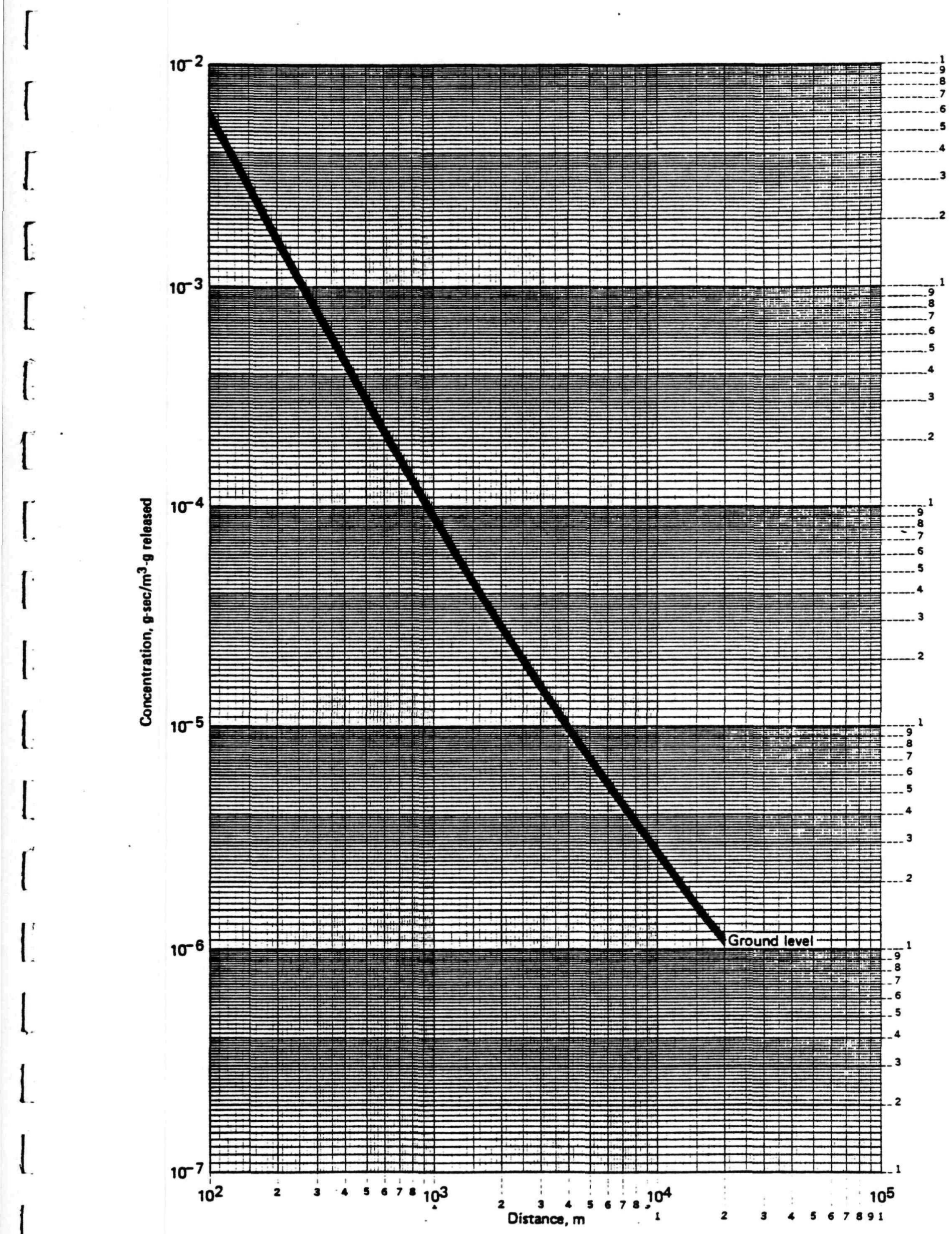

Figure 5-17. Integrated ground level airborne concentrations due to low altitude releases 

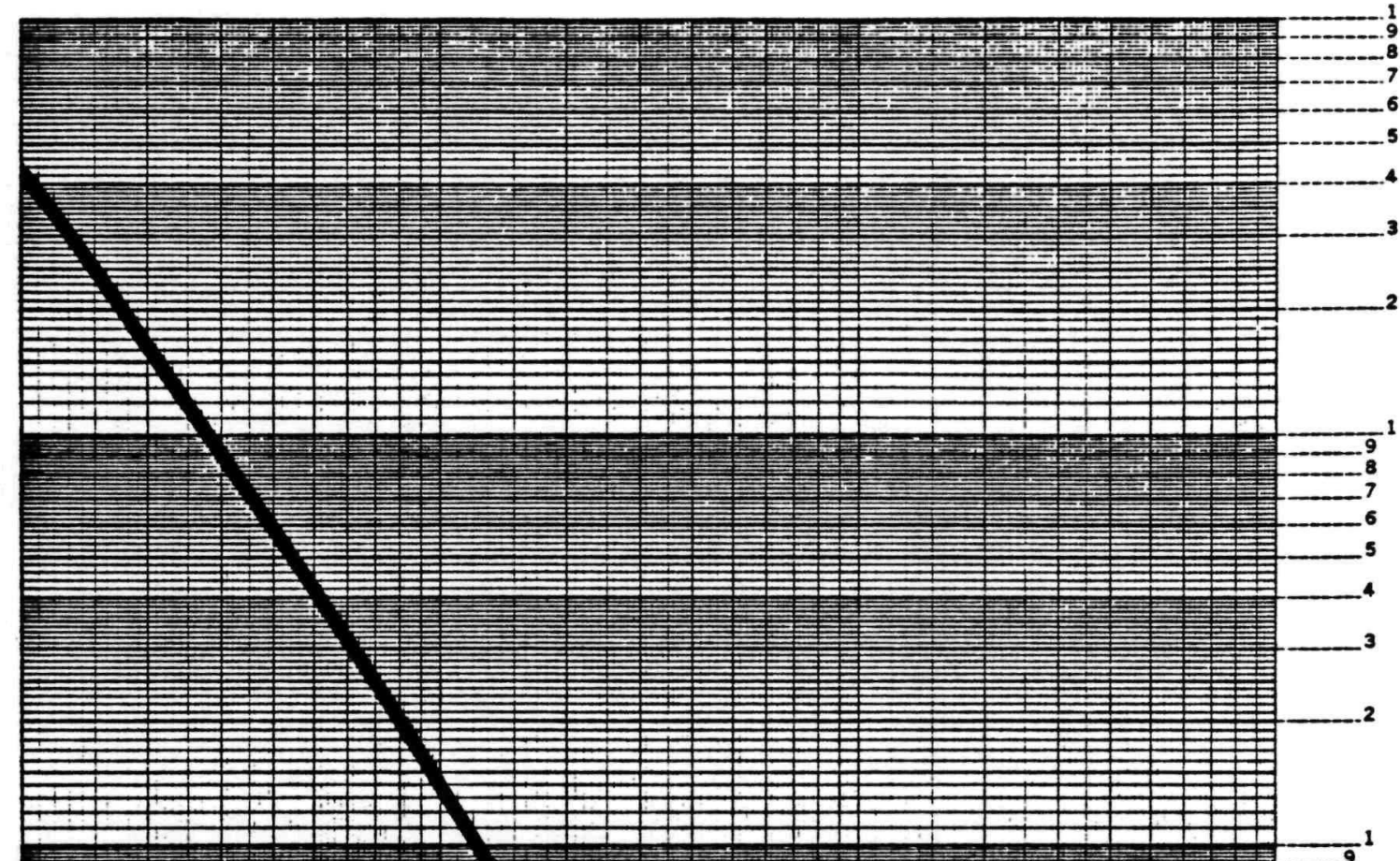
112 1

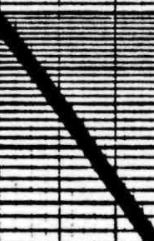

$10^{-6}$
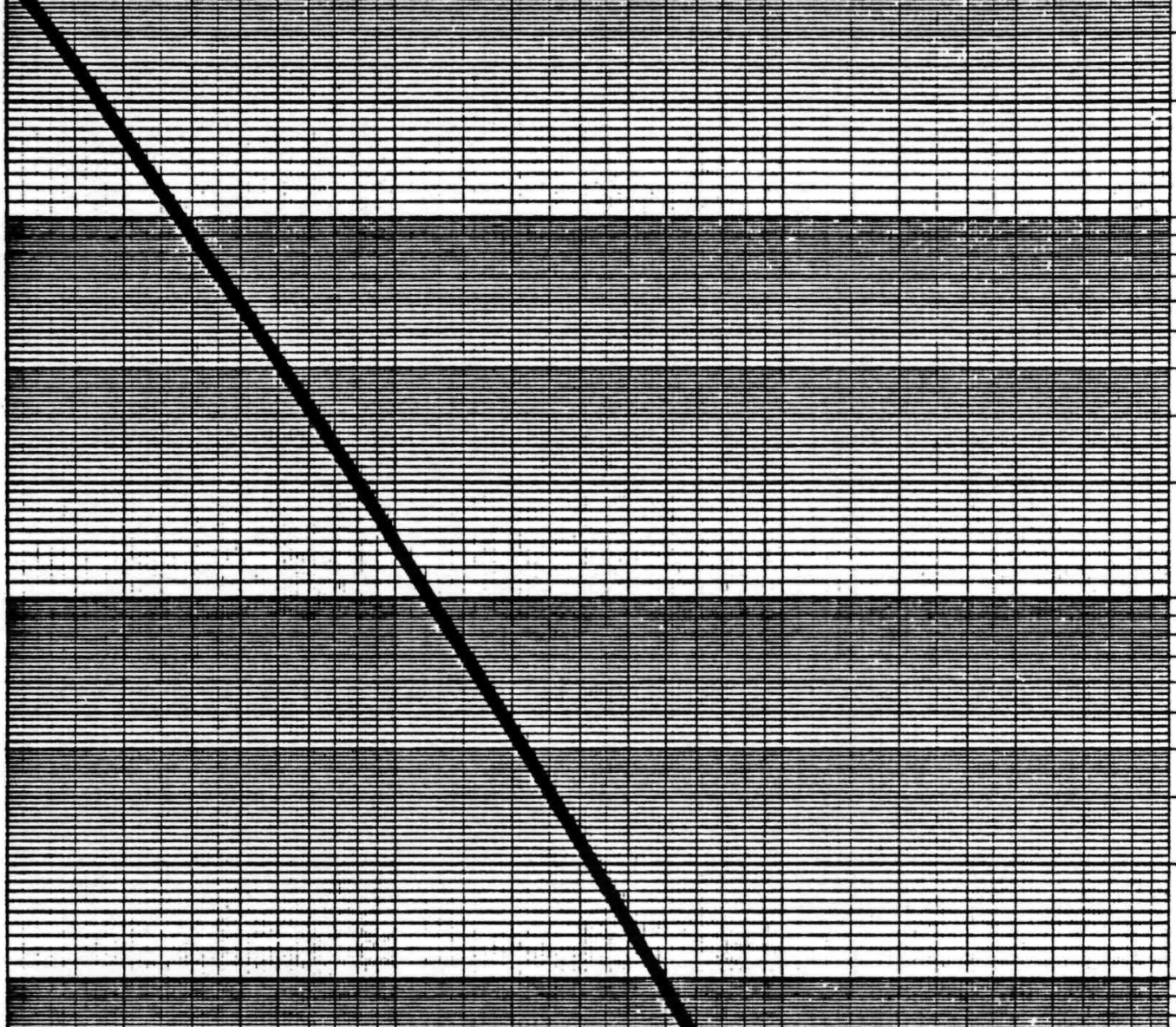

3

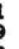




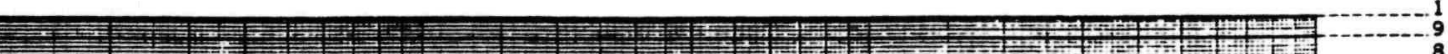

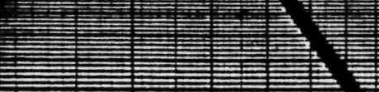

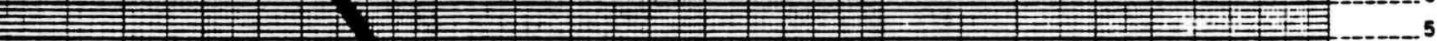
12171

$1+$
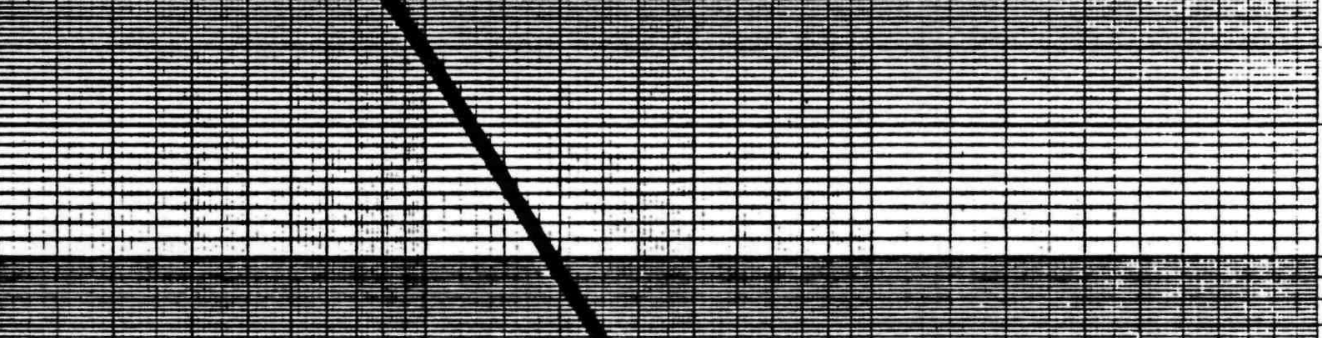

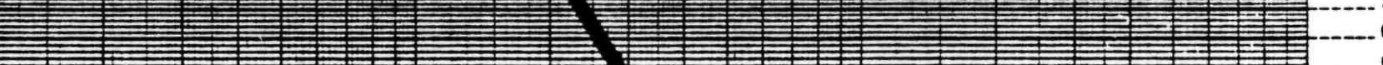
(1)
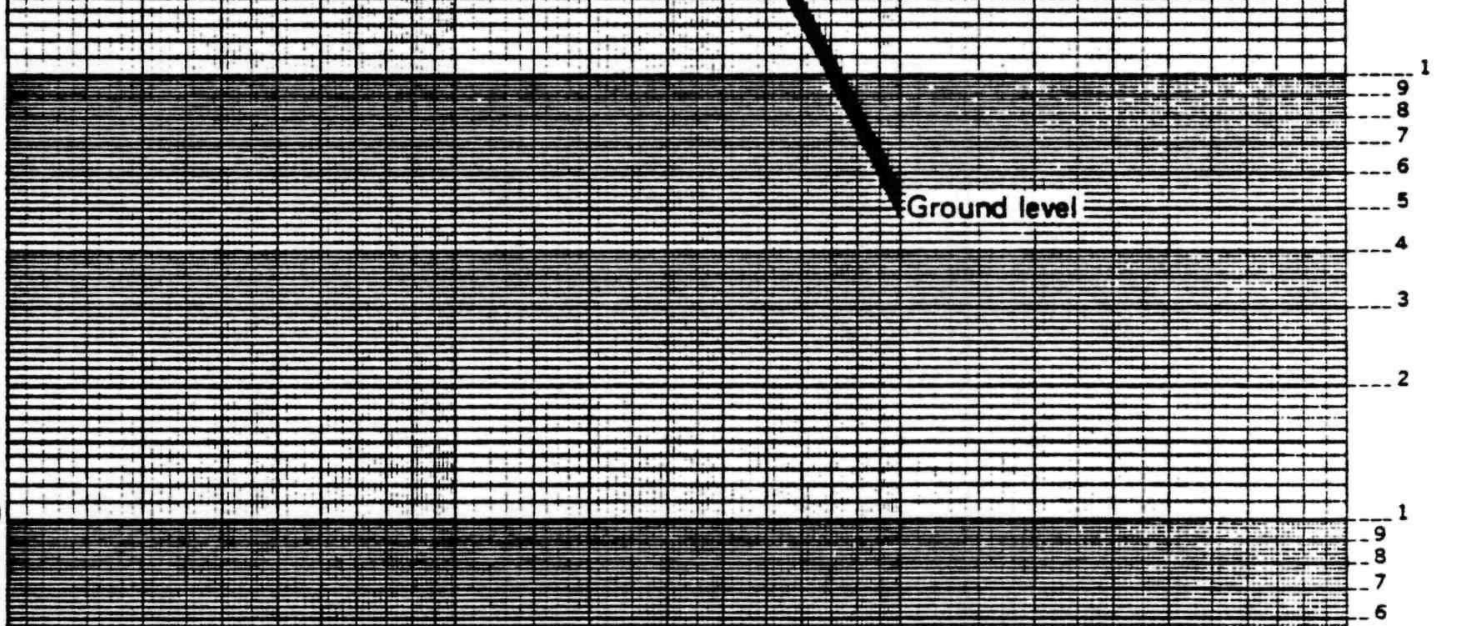

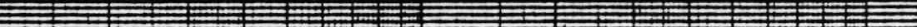

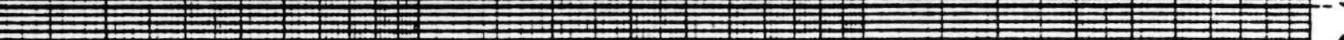

1

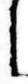

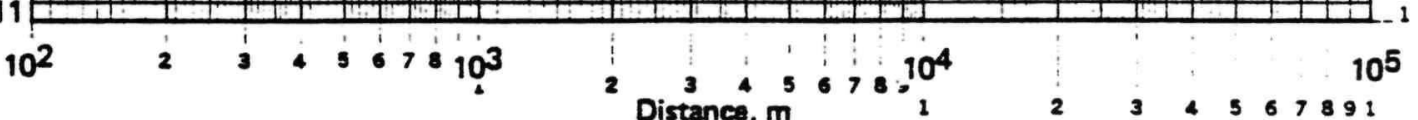

Figure 5-19. Vegetation concentrations due to low altitude releeses 
Using the mean value theorem of integral calculus,

$$
Q(0)=\bar{\omega}(0) A
$$

where

$$
\begin{aligned}
& \bar{\omega}(0)= \text { average integrated ground concentration over } \\
& \text { the affected area, } \mathrm{g} / \mathrm{m}^{2}
\end{aligned}
$$

The average time integrated airborne concentration due to initial deposition over the affected area can be represented as

$$
\bar{\psi}(0) \quad=\frac{0(0)}{\nabla}
$$

where

$$
\begin{aligned}
\psi(0)= & \begin{array}{l}
\text { average time integrated airborne concentration } \\
\text { over the affected area } \mathrm{A}, \frac{\mathrm{g}-\mathrm{sec}}{\mathrm{m}^{3}}
\end{array} \\
\dot{v}= & \text { effective deposition velocity, } \mathrm{m} / \mathrm{sec}
\end{aligned}
$$

Based on several studies in which airborne concentrations and ground deposition rates of radioactivity from low altitude atmospheric releases were measured simultaneously, the effective deposition velocity was assumed to be on the order of $0.01 \mathrm{~m} / \mathrm{sec}$.

In addition to the integrated airborne concentration due directly to the initial release, resuspension of ground deposited radioactive particulates will result in an added contribution. The resuspended airborne concentration at any time $t$ from Equation 5-35 can be written as

$$
\begin{aligned}
x_{R}(t) & =R(t) \omega(t) \\
& =R(t) \omega(0) e-\lambda_{w}=
\end{aligned}
$$

where the terms were defined previously. 
The integrated resuspended airborne concentration is

$$
\bar{\psi}_{R} \quad=\int_{0}^{\infty} R(t) \bar{\omega}(t) d t
$$

The total integrated airborne concentration is therefore,

$$
\begin{aligned}
\bar{\psi}_{I} & =\bar{\psi}(0)-\bar{\psi}_{R} \\
& =\frac{\omega(0)}{V}+\int_{0}^{\infty} R(t) \bar{\omega}(:) \dot{c} t \\
& =\frac{Q(0)}{A}\left[\frac{1}{V}+\int_{0}^{\infty} R(t) e^{-\lambda \omega t} d \tau\right]
\end{aligned}
$$

The integral has been developed further in section 5.1 .1 .2 and Equation 5-36.

The population exposure is

$$
\begin{gathered}
E=\rho A \psi T \\
I=\rho Q(0)\left[\frac{1}{V}+\int_{0}^{\infty} R(t) e^{-\lambda w t} d t\right]
\end{gathered}
$$

where

$$
\begin{aligned}
& E=\text { population exposure, persons }-g-y \Gamma / \mathrm{m}^{3} \\
& \rho=\text { population density, persons } / \mathrm{m}^{2}
\end{aligned}
$$

\subsection{AOUATIC DISPERSION MODELS}

Aquatic transfer processes include the interaction of airborne dispersed activity and intact point sources with water bodies such as rivers and streams, bays and large lakes, and oceans. These interactions include the dissolution of particulates and point sources, transfers to sediment, transfers to other water bodies, and transfers to food supplies such as fresh water food or seafood, as outlined in Figure 1-1. The aquatic transfer processes considered in this analysis are described below. 
The dissolution of fuel in the form of particulates or intact sources will be complex in nature, being a function of fuel encapsulation, chemical and physical form, and fuel geometry. The dissolution of fuel can be represented as shown in Figure 5-20. Encapsulated fuel in an aquatic environment is assumed to begin releasing dissolved fuel after time $t$, which is the time required for the encapsulation material to be dissolved. The quantity of dissolved fuel in solution as a function of time, referring to Figure 5-20, can be shown to be

$$
\begin{aligned}
g(t)=\frac{Q(0) \lambda_{d} s\left(t-t_{d}\right)}{\lambda_{f s}+\lambda_{d}-\lambda_{d s}}\left\{e^{-\left[\left(\lambda_{f s}-\lambda_{d s}\right) t_{0}+\lambda_{d s} t\right]}\right. \\
-e^{\left.-\left[\lambda_{f s}=-\lambda_{d}\left(=-t_{0}\right)\right]\right\}}
\end{aligned}
$$

where

$$
\begin{aligned}
s\left(t-t_{0}\right) & =\left\{\begin{array}{lll}
1 & \text { if } & t \geq t_{0} \\
0 & \text { if } & t<t_{0}
\end{array}\right. \\
t_{0} & =\begin{array}{l}
\text { time required for dissolution of encapsulation } \\
\text { material to occur, yrs }
\end{array}
\end{aligned}
$$

This formulation can represent a spectrum of aquatic release scenarios, ranging from the immediate release of the entire inventory $\left(t_{0} \rightarrow 0\right.$ and $\left.\lambda d \rightarrow-j\right)$, delayed release associated with the dissolution of very soluble fuel (large $\lambda d$ ), and the dissolution of very insoluble fuel (small $\lambda d$ ).

Studies of plutonium in aquatic environments indicate that dissolved fuef is transferred to the sediment sink with a 3.5 year half-Iife. (36) sediment sink in this case is taken to be that portion of sediment below the top $6 \mathrm{~cm}$. Therefore,

$$
\begin{aligned}
\lambda_{\mathrm{ds}} & =\frac{0.693}{3.5 \text { years }} \\
& =0.198 \mathrm{yz}^{-1}
\end{aligned}
$$

The $\lambda_{f g}$ in Figure 5-20 and Equation 5-46 represents the rate at which the intact fuel is removed from the aquatic environment by being covered with sediment or encrustation. The removal halflife associated with this removal mechanism has been somewhat arbitrarily taken to be 50 years, which is considered reasonable. 
$\boldsymbol{L}$

I

$\Gamma$

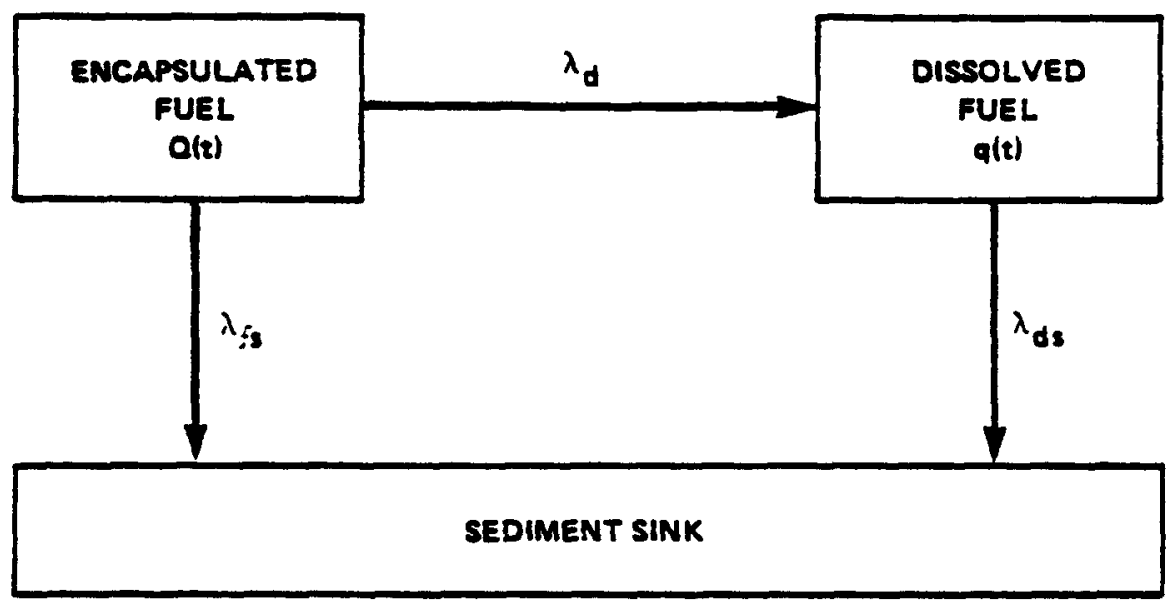

$Q(t)=$ QUANTITY OF FUEL INTACT AT TIME $t$, GRAMS

qIE = QUANTITY OF DISSOLVED FUEL IN SOLUTION AT TIME 2. GRAMS

$\lambda_{d}=$ FRACTION OF INTACT FUEL DISSOLVED PER UNIT TIME, y.'1

$\lambda_{f s}=$ FRACTION OF INTACT FUEL COVERED BY SEDIMENT PER UNIT TIME, Yr.

$\lambda_{\mathrm{ds}}$ - FRACTION OF DISSOLVED FUEL IN SOLUTION TRANSFERRED TO SEDIMENT SINK PER UNIT TIME, YP.-1

Figure 5-20. Diseolution of encapsulated fuel in aquatic environments. 
Bence,

$$
\begin{aligned}
\lambda_{\text {fs }} & =\frac{0.693}{50 \text { years }} \\
& =1.386 \times 10^{-2} \mathrm{yr}^{-1}
\end{aligned}
$$

The quantity of dissolved fuel $q(t) / Q(0)$ has been evaluated parametrically as a function of time for a range of $\lambda d$ and $t_{0}$ values, and the results are presented in Figure 5-21.

Particulate and vapor fuel dispersed atmospherically as a result of high or low altitude releases may be deposited on water surfaces. Equations 5-26 through 5-28 and 5-32, and Figure 5-14 for ground concentrations due to high altitude releases can be used directly to calculate the fuel transferred to water surfaces by multiplying by water surface area, determined using information presented in section 5.1.1.1. The same applies for low altitude releases, using the appropriate ground concentrations presented in Section 5.1.2.

It has been assumed that particulate and vapor fuel released atmospherically and deposited on the water surface are essentially in a dissolved state, corresponding to $t_{0}=0$.

The behavior of an operating reactor in water that could not be shutdown by command requires further evaluation and is not considered here.

The total amount of dissolved fuel can also be represented as

$$
q(t)=2 \pi \int_{0}^{R} c(t) r^{2} d r
$$

which fepresents the integration of the water concentration $c(t)$ in $\mathrm{g} / \mathrm{m}^{3}$ over a hemispherical volume extending out to some undefined radius $R$. Using the mean value theorem of integral calculus,

$$
\begin{aligned}
& q(t)=\bar{c}(t) \\
& \bar{c}(t)=\frac{g(t)}{V}
\end{aligned}
$$

where

$$
\begin{aligned}
\bar{c}(t) & =\begin{array}{l}
\text { average concentration of dissolved fuel in } \\
\text { water, } \mathrm{g} / \mathrm{m}
\end{array} \\
v & =\begin{array}{l}
\text { an arbitrary volume of water within which } \\
\text { the dissolved fuel is contained, } \mathrm{m}^{3}
\end{array}
\end{aligned}
$$


a

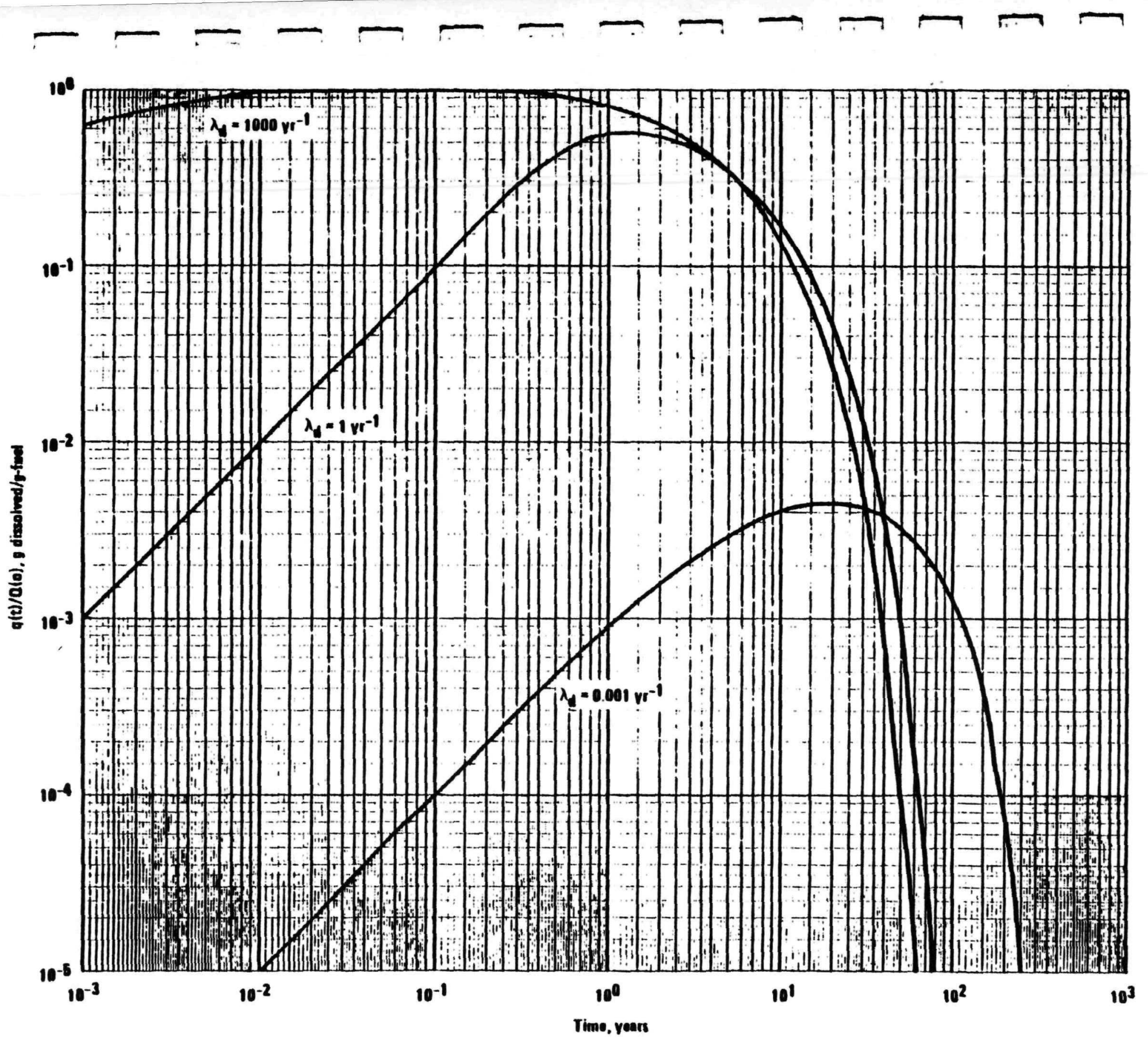

Figure 5-21. Dissolution of encapsulated fuel in aquatic environments as a function of time and dissolution rate. 
The interaction of the dissolved fuel with seafood, fresh water food, and drinking water must be considered in order to evaluate the resulting ingestion doses to people. The density of seafood caught annualiy in coastal waters has been estimated in Reference 2 to be

$$
\begin{aligned}
P_{f} & =8.613 \times 10^{-9} \mathrm{~g} / \mathrm{cm}^{3}-\mathrm{yr} \\
& =\text { density of caught seafood in coastal waters }
\end{aligned}
$$

The volume associated with the dissolved fuel will be taken as the elements of volume required to 1) produce an individual's annual requirement of seafood, and 2) to produce the annual requirement of seafood for the general population. The density of fresh water food in rivers, streams and lakes has been assumed to be the same as that for oceans. Impact probabilities of shallow ocean and fresh water have been presented in Table 5-2 and Figure 5-3. 


\subsection{RADIOLOGICAL DOSE MODELS}

Radiological dose models for both dispersed fuel and intact sources are presented in this section.

\subsection{RADIOLOGICAL DOSE MODELS FOR DISPERSED FUEL}

Radiation doses to both individuals and population groups have been calculated using the basic methods presented in OSNRC Regulatory Guide $1.109(22,37)$ and a Fortran Computer Code developed for this project by the Nos Corporation. The basic equation utilized in these calulations is as follows:

$$
D_{i j t}=\sum_{k} C_{k j t} \times \sigma_{j t} \times D C F_{k i}
$$

where

$$
\begin{aligned}
& D_{i j t}=\text { Dose to organ i from exposure to environmental } \\
& \text { medium } j \text { for time period } t \text {, mrem } \\
& \begin{aligned}
C_{k j t}= & \text { Average concentration of nuclide } k \text { in medium } j \\
& \text { for time period } t, \mathrm{pCi} / \mathrm{m}^{3}, \mathrm{pCi} / \mathrm{kg}, \mathrm{pCi} / 1, \mathrm{pCi} / \mathrm{m}^{2}
\end{aligned} \\
& \begin{aligned}
& U_{j t}= \text { Usage of environmental medium } j \text { by an individual } \\
& \text { over time period } t, \mathrm{~m}^{3}, \mathrm{~kg}, 1, \text { hours }
\end{aligned} \\
& D F_{i}=\text { Dose factor for nuclide } k \text { and organ } i \text {, mrem/pCi } \\
& \text { (inhaled or ingested), mrem/hr per } \mathrm{pCi} /\left(\mathrm{m}^{3} \text { or } \mathrm{m}^{2}\right. \text { ) }
\end{aligned}
$$

This equation is used to calculate exposure to either a maximum exposed individual or the average individual in a population group. Multiplying the average individual dose by the population at risk and a factor of 0.001 for units conversion yields population dose in manrem. The computer code uses population dose to estimate health effects as discussed in Chapter 7.

Eight exposure pathways are considered in this analysis. They are:

Air Inhalation
Air Immersion
Ground Shine
Vegetation Ingestion

Meat Ingestion

Milk Ingestion

Water Ingestion

Aquatic Foods Ingestion

Initially, the concentrations of each nuclide for each time period and environmental media is determined. Air concentrations are determined for both the inhalation and immersion exposure pathway as follows:

$$
C A_{k t}=\frac{X(0, t)}{Q(0)} \times Q(0) \times S P A_{k t} / \Delta t
$$


where

$$
\begin{aligned}
& C A_{k t}=\text { Average concentration of nuclide } k \text { in air for } \\
& \text { time period } t, \mathrm{pci} / \mathrm{m}^{3} \\
& \underline{x(0, t)}=\text { Time integrated normalized mass concentration } \\
& Q(0) \text { of released fuel in air at ground level for } \\
& t \text { ime period } t, g-s e c / m^{3} \text { per } g \text { of fuel released } \\
& \text { (Section 5.1) } \\
& Q(0)=\text { Mass of fuel released at time } 0, g \\
& \text { SPA }{ }_{k t}=\text { Specific activity of nuclide } k \text { in the released } \\
& \text { fuel at time } t, \mathrm{pCi} / \mathrm{gm} \text { (Chapter 4) } \\
& \Delta t=\text { Length of time step } t \text {, sec }
\end{aligned}
$$

Ground surface deposition is used in determining ground shine doses. Total ground surface deposition is determined as follows:

$$
{C G_{k t}}=\frac{\omega(t)}{Q(0)} \times Q(0) \times \text { SPA }_{k t}
$$

where

$$
\begin{aligned}
C G_{k t}= & \begin{array}{l}
\text { Average deposition of nuclide } k \text { at } t \text { ime } t, \\
\mathrm{PCi} / \mathrm{m}^{2}
\end{array} \\
\frac{\omega(t)}{Q(0)}= & \begin{array}{l}
\text { Normalized mass deposition of released fuel on } \\
\text { ground surfaces at } t \text { ime } t, g / \mathrm{m}^{2} \text { per } g \text { of fuel } \\
\text { released }(5-1)
\end{array}
\end{aligned}
$$

The mass deposition in this equation considers all fuel deposited on ground surfaces from time 0 , and weathering removal.

Released radionuclides in and on vegetation can result in human exposure from direct ingestion of vegetation or the ingestion of animal products from animals exposed to this vegetation. Radionuclide concentrations in three types of vegetation are calculated: vegetables, pasture grass and stored animal feed. Two pathways for contamination of vegetation are considered as follows:

$$
C v_{k t v}=v r_{k t v}+v R_{k t v}
$$

where

$$
\begin{aligned}
C V_{k t v}= & \text { Total concentration in vegetation type } v \text { radio- } \\
& \text { nuclide } k \text { at time } t, p c i / k g \\
V_{k t v}= & \text { Portion of the total vegetation concentration } \\
& \text { of nuclide } k \text { at time } t \text { contributed by foliar } \\
& \text { deposition, pci/kg }
\end{aligned}
$$




$$
\begin{aligned}
V_{k t v}= & \text { Portion of the total vegetation concentration on } \\
& \text { nuclide } k \text { at time } t \text { contributed by root uptake, } \\
& p c i / k g
\end{aligned}
$$

The foliar deposition portion is determined by:

$$
V_{k t v}=\frac{\frac{C_{v}(t)}{Q(0)} \times Q(0) \times \operatorname{SPA}_{k t}}{Y_{V}} \times e^{-\lambda_{k} t_{h v}}
$$

where

$$
\begin{aligned}
\frac{C_{v}(t)}{Q(0)}= & \begin{array}{l}
\text { Normalized mass deposition of released fuel } 0 \\
\text { vegetation surfaces at } t \text { ime } t, \mathrm{~g} / \mathrm{m}^{2} \text { per } \mathrm{g} \text { fuel } \\
\\
\text { released (Section } 5.1 \text { ) }
\end{array} \\
Y_{v}= & \text { Yield of vegetation type } v, \mathrm{~kg} / \mathrm{m}^{2}(0.75 \text { for } \\
& \text { pasture, } 2.0 \text { for feed and vegetables) } \\
\lambda_{k}= & \text { Radiological delay constant for nuclide } \mathrm{k}, \mathrm{l} / \mathrm{sec} \\
t_{\mathrm{h} v}= & \text { Time from harvest of vegetation type } v \text { to con- } \\
& \text { sumption by man or animals, sec (Equivalent } \\
& \text { to } 0 \text { days for pasture, } 90 \text { days for feed and } \\
& 14 \text { days for vegetables) }
\end{aligned}
$$

The root uptake portion is determined by:

$$
v R_{k t n}=\frac{\omega(t)}{Q(0)} \times Q(0) \times \operatorname{SPA} k t \times B_{k v} \times e^{-\lambda_{k} t_{h v}} \times \frac{1}{\bar{P}}
$$

where

$$
\begin{aligned}
B_{k v}= & \text { Bioaccumulation factor for vegetation for nuclide } \\
& k, \text { pCi } / \mathrm{kg} \text { vegetation per } \mathrm{pCi} / \mathrm{kg} \text { soil (from Reference } \\
& 38, \text { see Table } 6-1) \\
P= & \text { Product of soil density and a plow layer depth of } \\
& 6 \text { inches, } \mathrm{kg} / \mathrm{m}^{2}\left(240 \mathrm{~kg} / \mathrm{m}^{2}\right)
\end{aligned}
$$

The amount of a radionuclide which appears in animal products, either meat or milk is a function of the amount of a radionuclide in the animal's diet. This concentration is determined from the concentrations in pasture and feed and the relative contribution of these two dietary components as follows:

$$
C V_{k t d}=F_{p} F_{s} C V_{k t p}+\left(1-F_{p}\right) C V_{k t s}+F_{p}\left(1-F_{s}\right) C V_{k t s}
$$

where

$$
\begin{aligned}
& F_{P}=\text { Fraction of the year animals consume pasture } \\
& F_{s}=\text { Fraction of the animal's diet derived from pasture } \\
& \text { during the pasture season }
\end{aligned}
$$


Table 6-1. Dose Calculations Blotransfer Factors

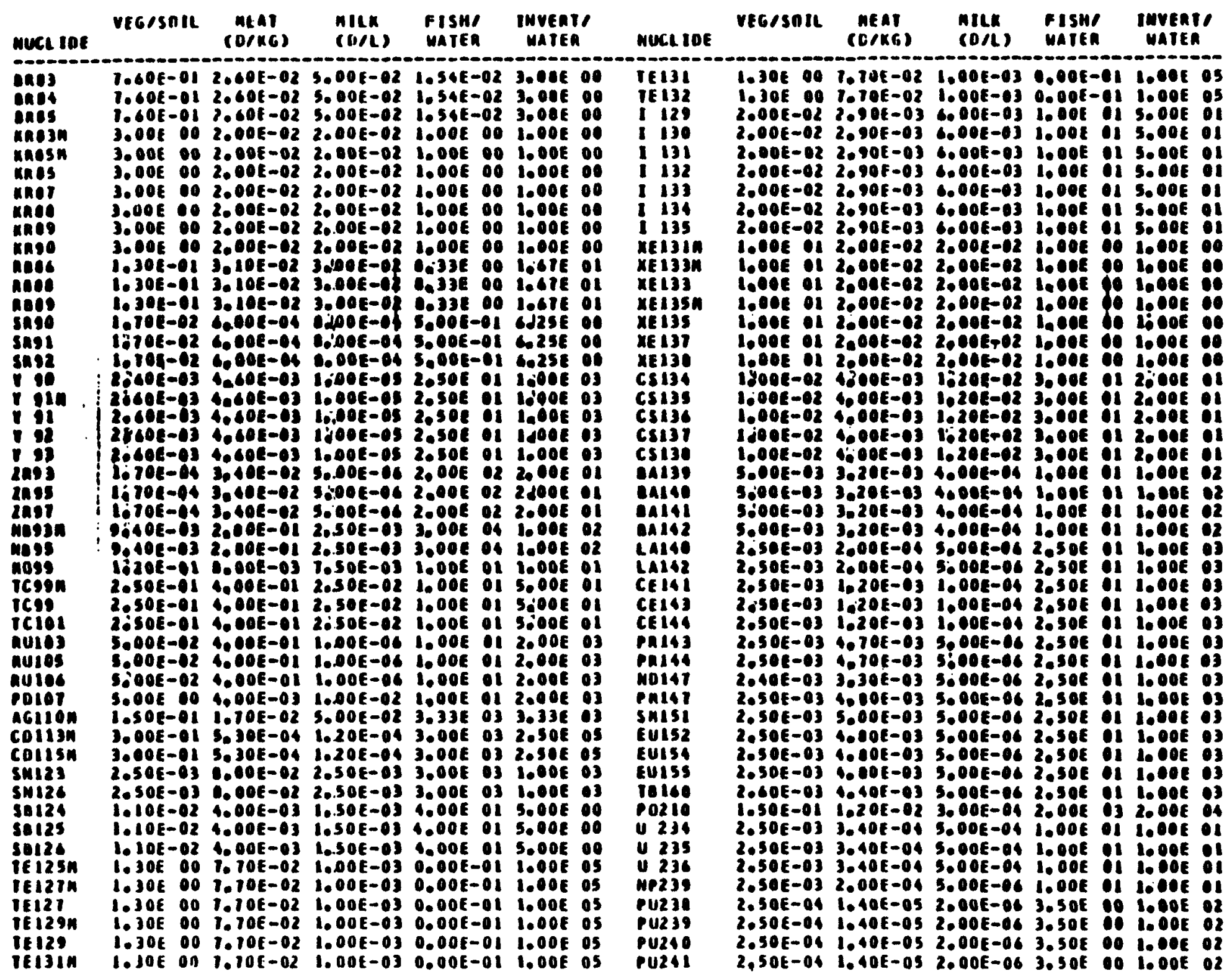


The subscripts of the $c v$ term $d, p$, and $s$ refer to the total diet, pasture, and stored feed, respectively.

The concentration in meat and milk is then determined by:

$$
M_{k t m}=B_{k m} \times C V_{k t d} \times I N_{m} \times e^{-\lambda_{k} t} s m
$$

where

$$
\begin{aligned}
& M_{k t m}=\text { Concentration of radionuclide } k \text { at } t \text { ime } t \text { in animal } \\
& \text { product m, pCi/kg or } \mathrm{pCi} / 1 \\
& B_{k m}=\text { Biological transfer rate of consumed radionuclide } k \\
& \text { to animal product } \mathrm{m} \text {, days } / \mathrm{kg} \text { or days } / \mathrm{l} \text {. (Table 6-I) } \\
& I N_{m}=\text { Daily intake rate of food by animal type } \mathrm{m}, 150 \mathrm{~kg} / \\
& \text { day for meat and milk animals) } \\
& t_{\text {sm }}=\text { Time from animal product m collection to consumption } \\
& \text { by man, sec (equivalent to } 20 \text { days for meat and } 4 \\
& \text { days for milk) }
\end{aligned}
$$

Buman exposures through aquatic pathways can occur via the consumption of contaminated potable water or aquatic foods living in contaminated water. Other aquatic dose pathways such as swimming and boating are thought to be inconsequential. The concentration of radionuclides in potable water is determined by:

$$
w_{k t}=\frac{\bar{C}(t)}{Q(0)} \times Q(0) \times \operatorname{SPA}_{k t} \times e^{-\lambda_{k} t_{p}} \times 0.001
$$

where

$$
\begin{aligned}
& w_{k t}=\text { Concentration of nuclide } k \text { in potable water at time } \\
& t, p C i / l \\
& \bar{C}(t)=\text { Normalized mass concentration of released fuel in } \\
& \overline{Q(0)} \text { water at } t \text { ime } t, g / \mathrm{m}^{3} \text { per } g \text { fuel released (Section } 5.2 \text { ) } \\
& t_{p}=\text { Time from extraction of water from a water body } \\
& \text { to consumption of the water by man, sec (equiva- } \\
& \text { lent to } 24 \text { hours) } \\
& 0.001=\text { Units conversion }=\mathrm{m}^{3} / 10001
\end{aligned}
$$


The concentrations of radionuclides in these aquatic food types are determined by:

$$
A_{k t a}=\frac{\bar{C}(t)}{Q(0)} \times Q(0) \times \operatorname{SPA}_{k t} \times B_{k a} \times e^{-\lambda_{k} t_{q} \times 0.0001}(6-10)
$$

where

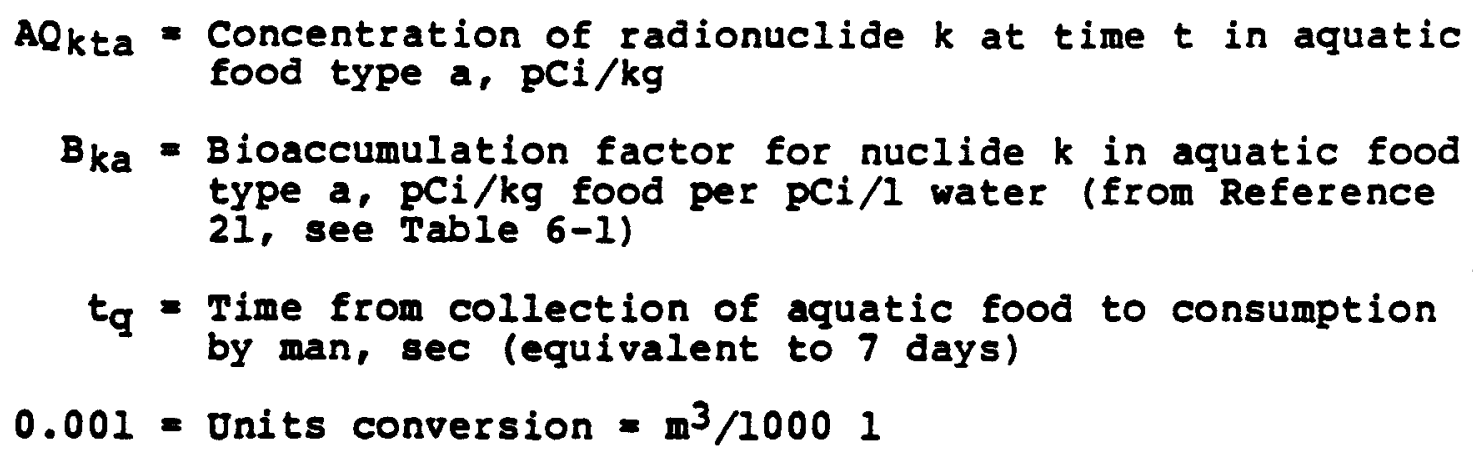

The model equations given above for calculating concentrations of $r$ adionuclides in biological medium (vegetation, meat, milk, and aquatic foods) include derived biological transfer parameters. These parameters assume that environmental medium concentrations are relatively stable for long periods (up to 1 year) so that equilibrium between the medium and the biological species can be achieved. The user of this model may select very short time periods for dose calculations. The model assumes that equilibrium between the environmental medium and all biological species is achieved instantaneously. This assumption will result in some inaccuracy in estimating biological medium concentrations for short time periods. Bowever, these inaccuracies will be minimal when concentrations and doses are integrated over long time periods. Caution must be used when interpreting dose rates from the consumption of foods in which concentrations have been determined for short time periods (less than 1 month).

The second term in equation $6-1$ is the usage of each environmental pathway. These usages are presented on an annual basis in Table 6-1. When calculating doses, these usage rates are scaled to the length of the time period involved. The usage rates used in this model are considered typical for the world's population. For the air immersion and ground shine pathways, continuous exposure is assumed with no credit for structural (Dwelling) shielding. The reference for each usage term is also given in Table 6-1.

The last term in equation $6-1$ is the dose factors. The dose factors are presented in Table 6-2. The inhalation and ingestion dose factors are actualiy dose commitment factors (DCF) and were obtained from OSNRC report NUREG-0172 (37). Once radionuclides are taken into the body, they can stay in the body for many years; being removed by normal biological functions at various rates. Thus, radionuclides taken into the body today 
Table 6-2. Osage Rates for Various Exposure Pathways

\begin{tabular}{|c|c|c|c|}
\hline Batbray & Dasage & Units & Reference \\
\hline $\begin{array}{l}\text { Inbalation } \\
\text { Air immersion } \\
\text { Ground shine }\end{array}$ & $\begin{array}{l}8000 \\
8760 \\
8760\end{array}$ & $\begin{array}{l}\mathrm{m}^{3} / \mathrm{yr}^{2} \\
\mathrm{hr} / \mathrm{yr} \\
\mathrm{hr} / \mathrm{yr}\end{array}$ & $\begin{array}{r}6 \\
22 \\
22\end{array}$ \\
\hline $\begin{array}{l}\text { Conswoption of } \\
\text { Vegetation } \\
\text { Meat } \\
\text { Milk* } \\
\text { Water } \\
\text { Aquatic foods ** }\end{array}$ & $\begin{array}{l}285 \\
9.8 \\
98.8 \\
370 \\
17.7\end{array}$ & $\begin{array}{l}\mathrm{kg} / \mathrm{yr} \\
\mathrm{kg} / \mathrm{yr} \\
1 / \mathrm{yr}^{2} \\
\mathrm{l} / \mathrm{yr} \\
\mathrm{kg} / \mathrm{yr}\end{array}$ & $\begin{array}{l}6 \\
6 \\
6 \\
6 \\
6\end{array}$ \\
\hline
\end{tabular}

- Includes a density conversion of $1.032 \mathrm{~kg} / \mathrm{l}$.

-728 Eish and 28 invertebrate species. 
Table 6-3. Dose Calculations Dose Factor Library

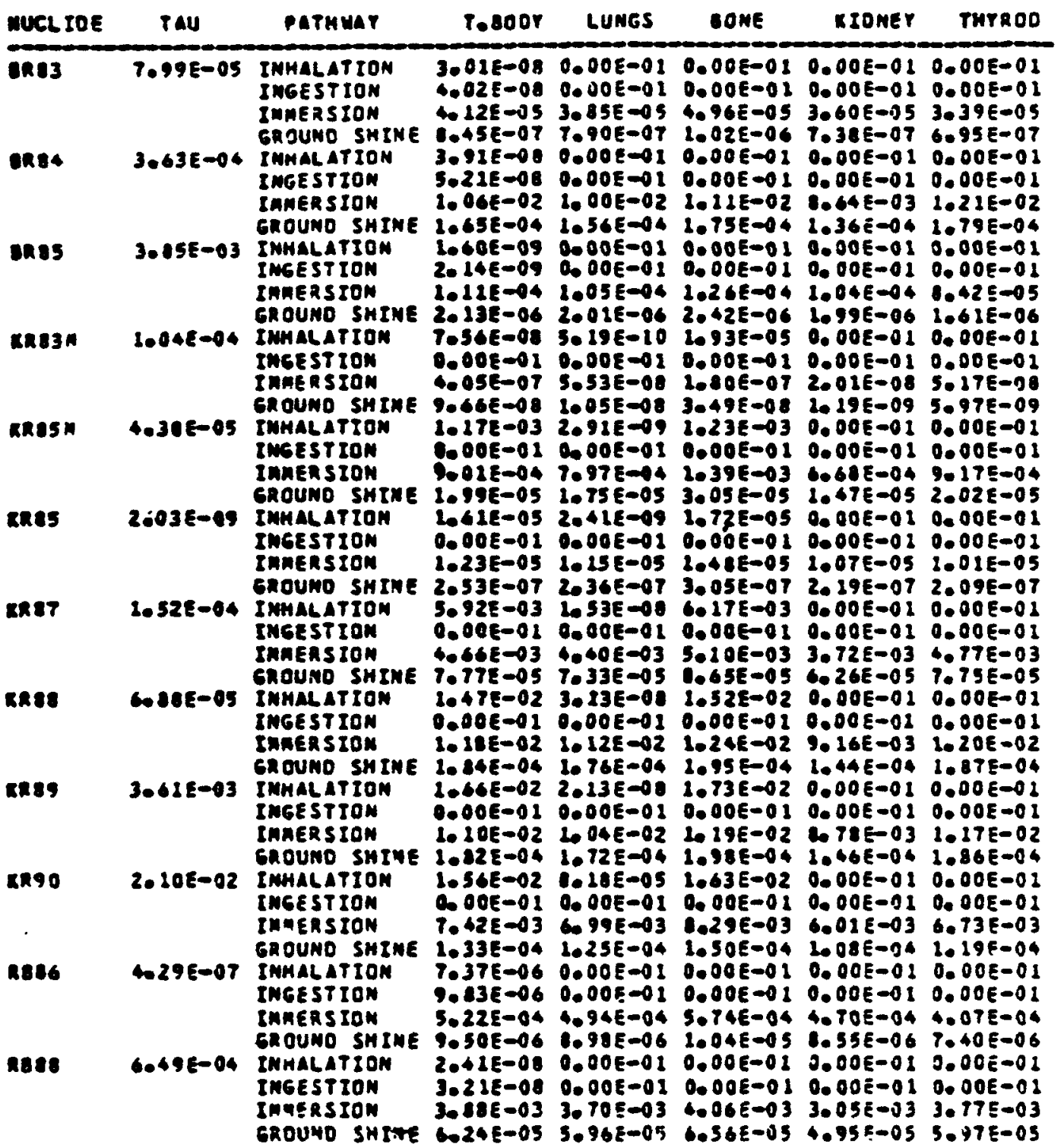

LeT To DOSE Facton GIERART

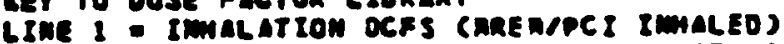

LINE 2 - INGESTION DCFS (AREAPPCI TWEESTED)

LIME 3 - INAERSION OCFS (MREMTTEAR PER DCIJMEAs)

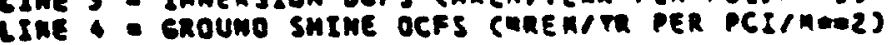


Table 6-3. Dose Calculations Dose Factor Library (Continued)

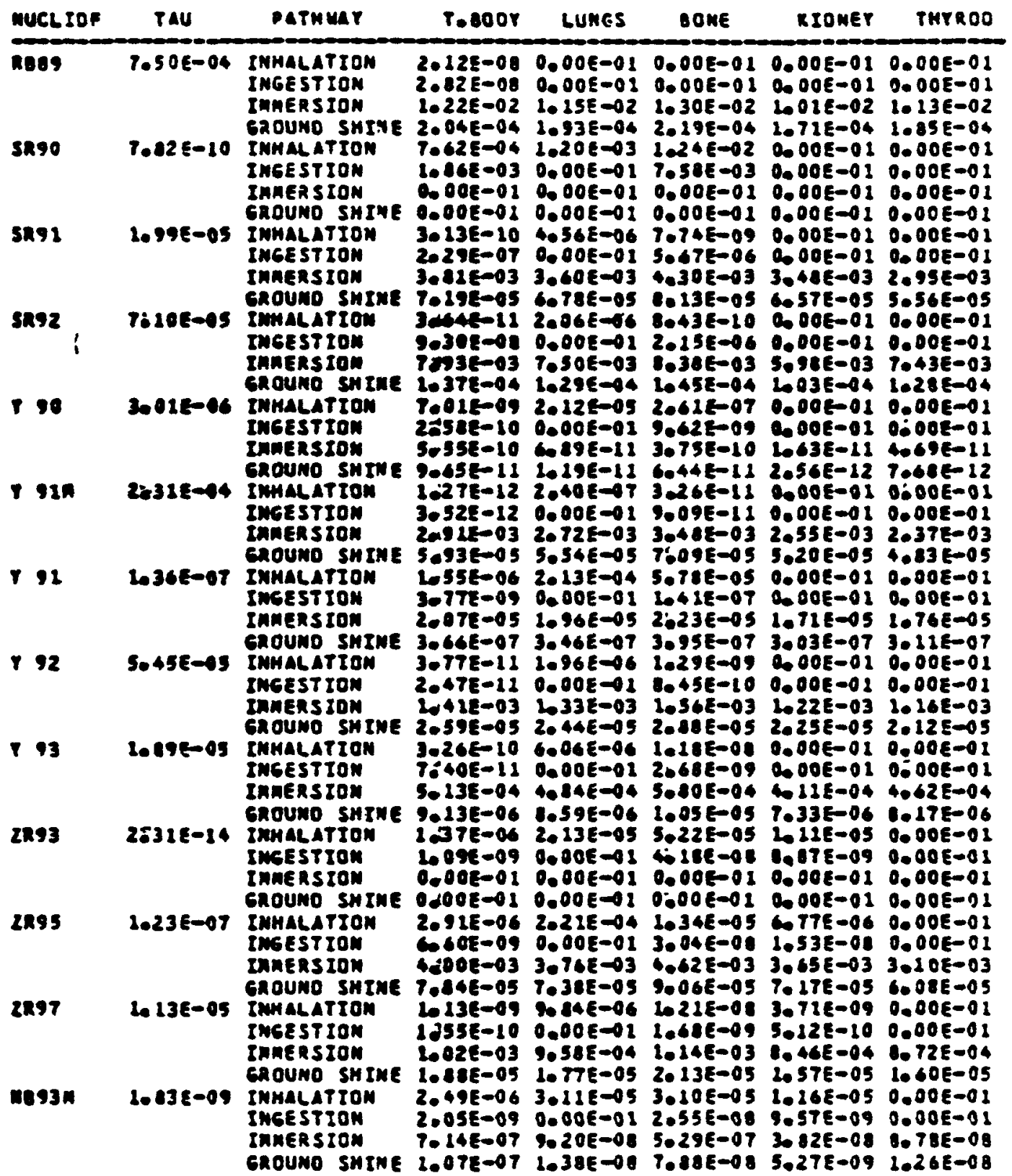


Table 6-3. Dose Calculations Dose Factor Library (Continued)

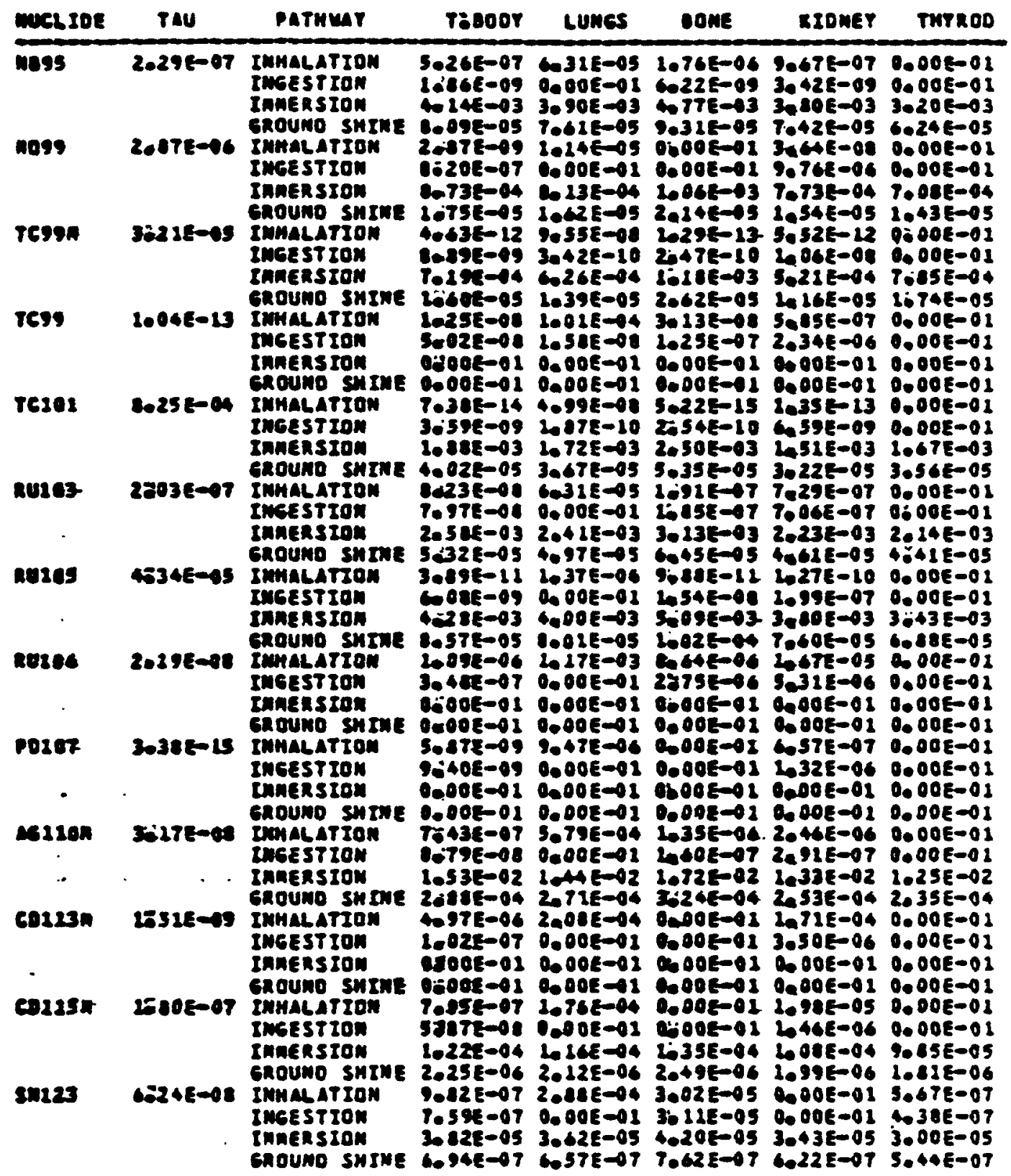


Table 6-3. Dose Calculations Dose Factor Iibrary (Continued)

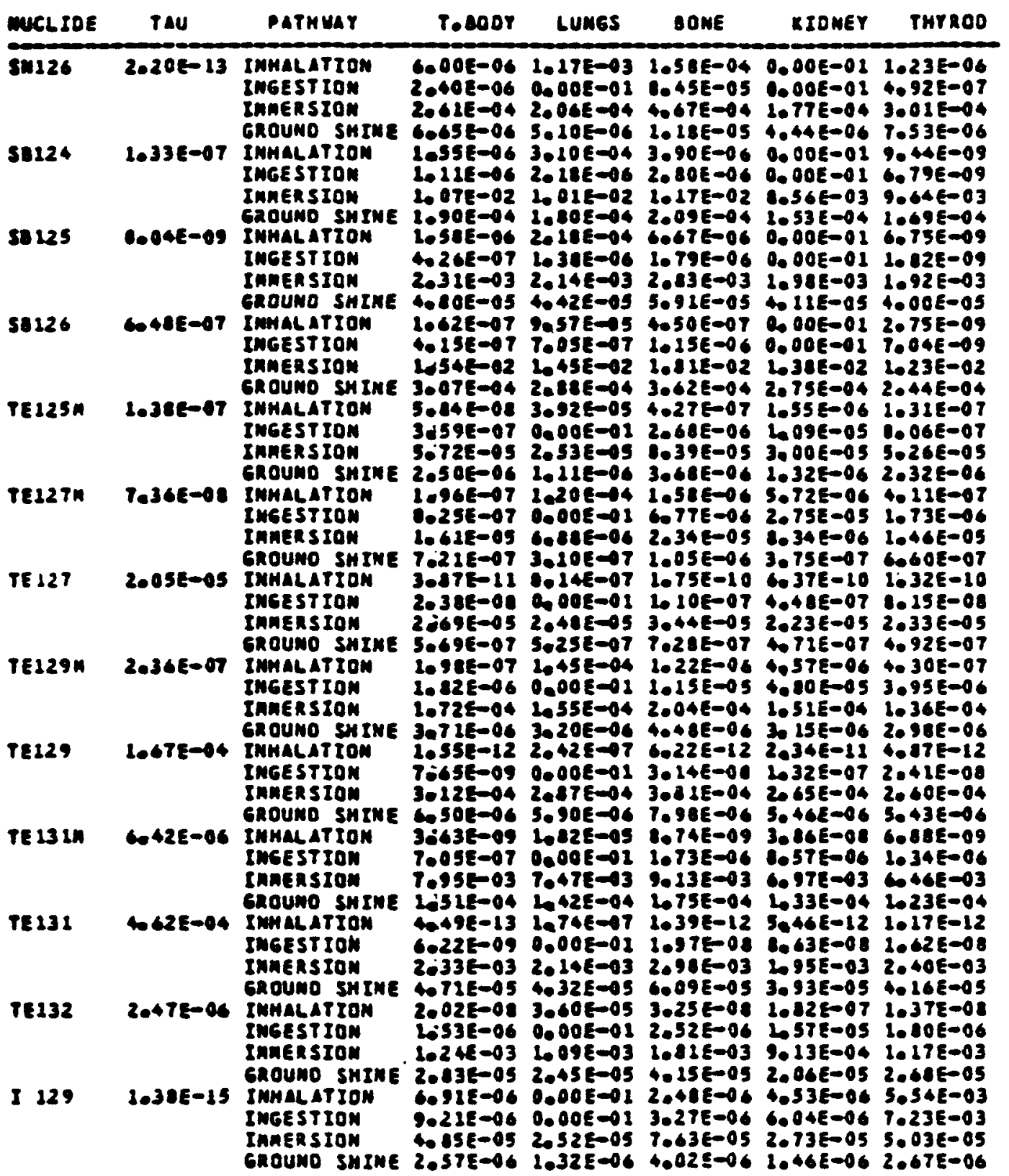


Table 6-3. Dose Calculations Dose Factor Iibrary (Continued)

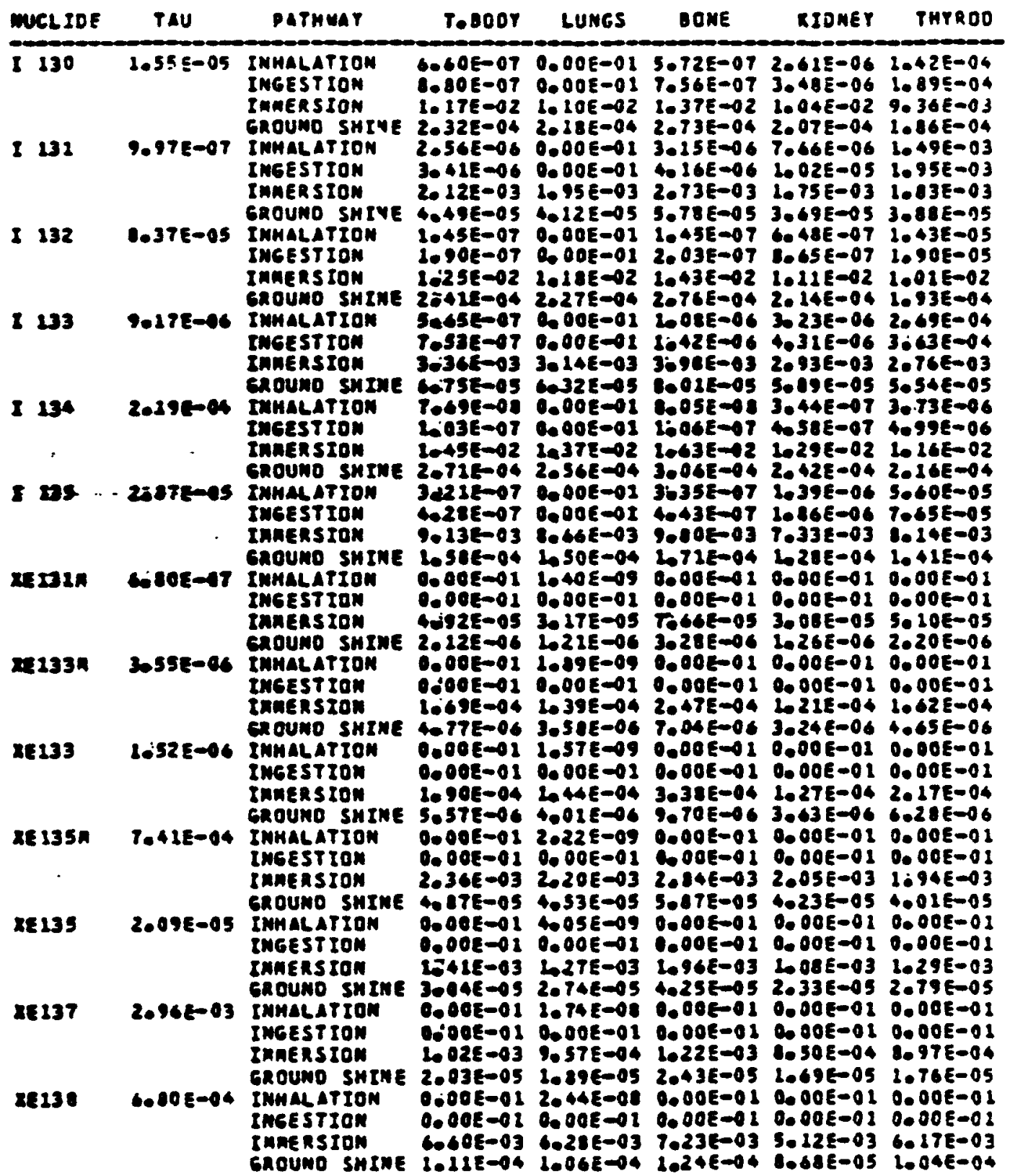


Table 6-3. Dose Calculations Dose Factor Iibrary (Continued)

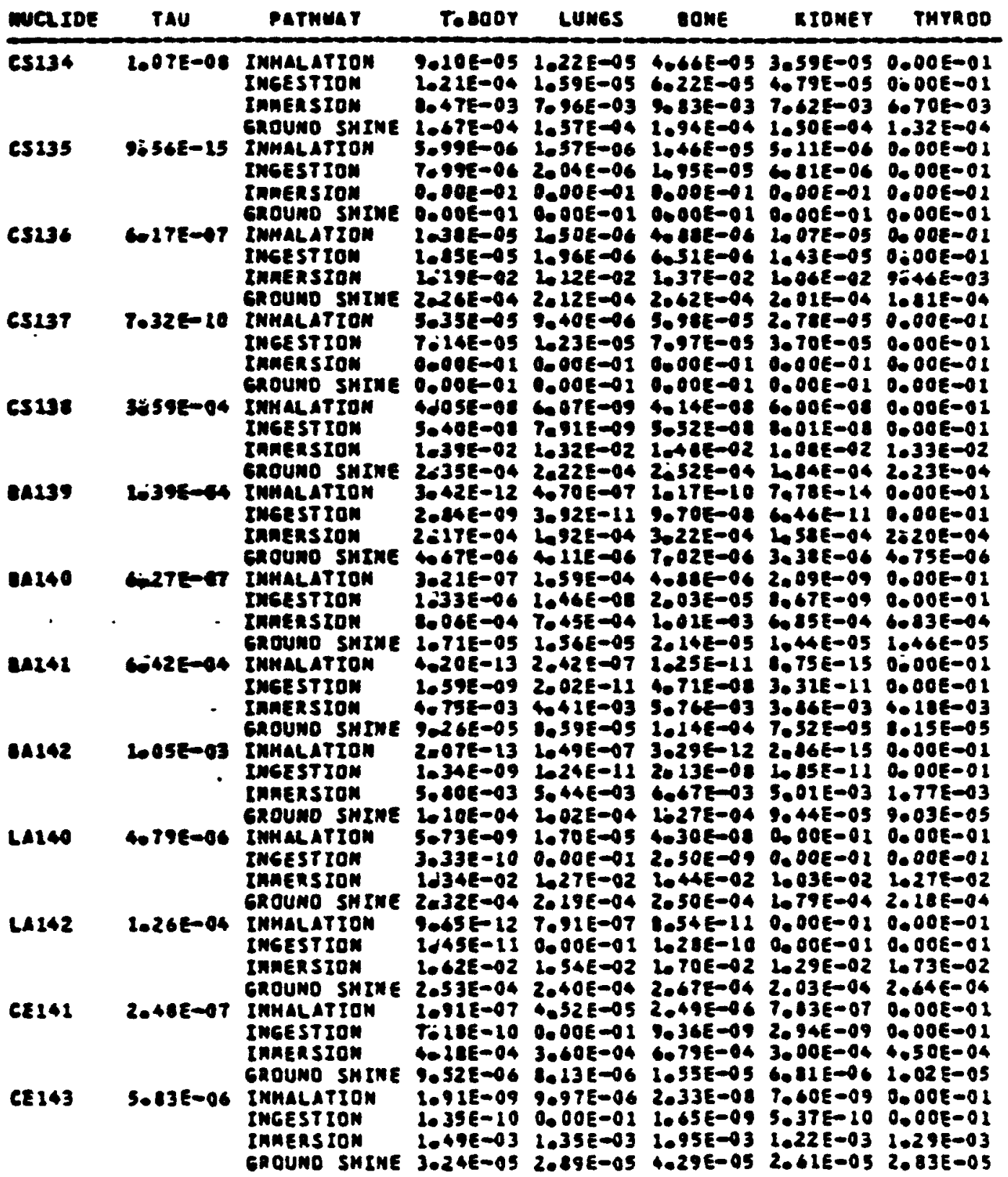


Table 6-3. Dose Calculat1ons Dose Factor Library (Continued)

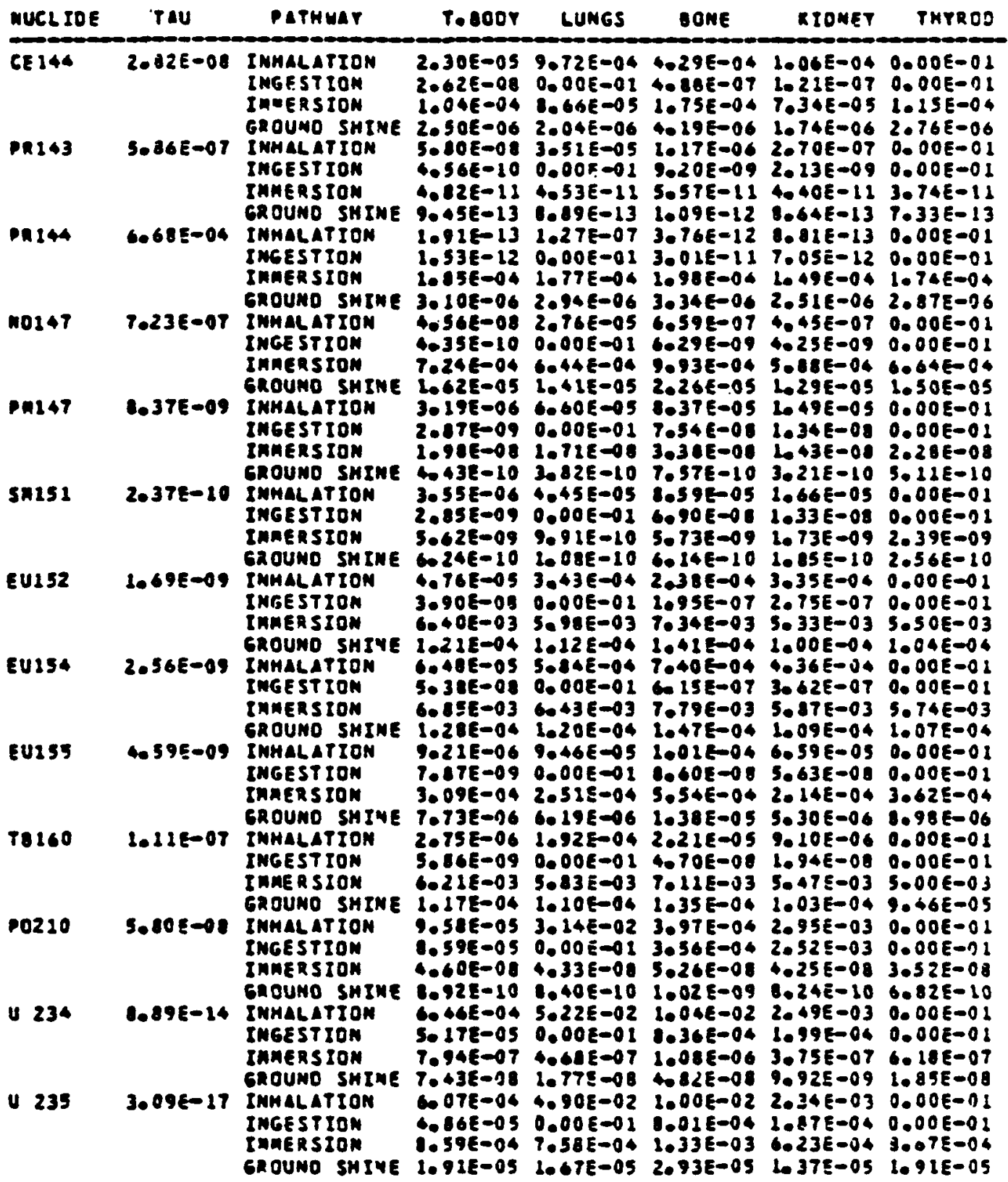


Table 6-3. Dose Calculations Dose Factor Iibrary (Continued)

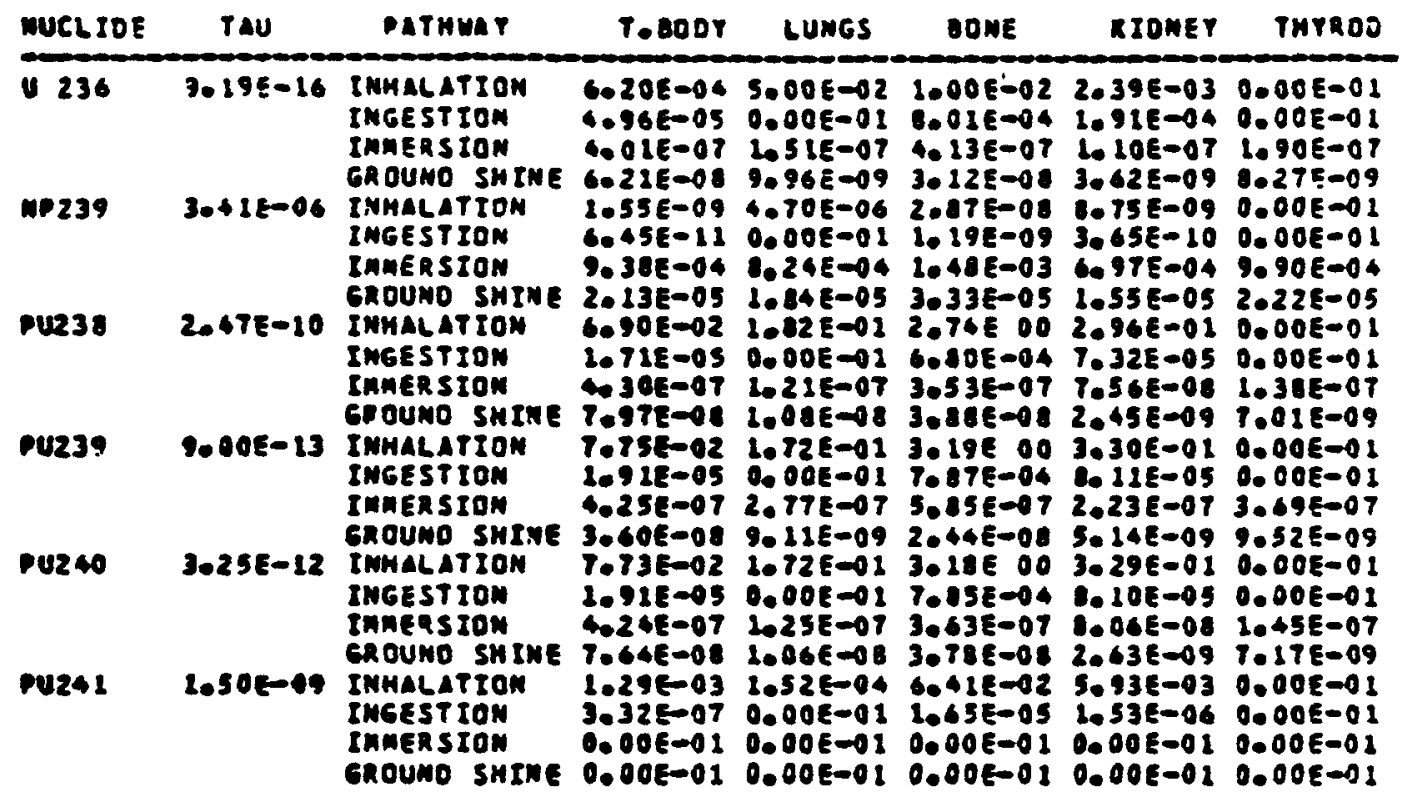

REY TO DOSE FACTOR LTERARY

LIWE 1 - INHALATION DCFS (AREM/PCI IWMALEO)

IIHE 2 - INGESTION DCFS (AREMTPET TMGESTED)

LIME 3 - IAMERSTON DCFS (MREMTYEAR PER DCI/MEN3)

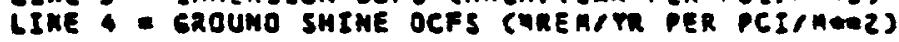


could be delivering doses 50 years from now. The DCF used in this analysis account for all doses to an individual for 50 years resulting from a single intake. The external dose factors (air immersion and ground shine) were developed by Rocher (39) and are in units of mrem/yr per $\mathrm{pCi} / \mathrm{m}^{2}$. These factors actually include an assumed usage rate of 8760 hours per year.

Five organs where considered in this analysis: total body, lungs, bone, kidneys, and liver. Dose factors for each type of exposure and for each organ are included in a dose factor library.

The computer code developed to implement the above model was prepared using the FORTRAN language. The user of the code supplies two run-specific input files. The first contains the nuclide name (e.g. XEI3IM) and specific activities for each of up to 10 time steps for each nuclide to be considered. The specific activities consider only radioactive decay and daughter nuclide ingrowth with time. The second file contains the balance of the user-supplied input. Included is the mass air concentration, ground deposition, foliar deposition, water concentration, and length in seconds for each time step. Other inputs include the grams of fuel originally released, the population at risk, health risk conversion factors for the five organs, the values of $F_{p}$ and $F_{S}$, and a key to control the calculation of potable water consumption doses.

The code operates in three modes depending on the user-supplied value for the population at $r$ isk (P). If a value of 1 is entered, the code computes individual dose reported in units of mrem. If a value of 0 or greater than 1 is entered, the code computes population dose (in man $r e m$ ) and health effects as expected premature cancer deaths. If the value is 0 , a population of 1 is assumed and the results can be scaled to any population size. A value greater than 1 is assumed to be the actual population at $r$ isk.

Code output includes tabular output as follows:

1. User-supplied imput parameters

2. Oser-supplied specific activities by nuclide and time

3. Dose factor library

4. Bio-transfer parameters

5. Dose or health effects by nuclide and time for each organ with all pathways summed

6. Dose or health effects by nuclide and organ for each time period with all pathways summed

7. Dose or health effects by nuclide and pathway integrated over a 1 year period for each organ 
8. Dose or health effects by organ, pathway and time with all nuclides summed

When operating in the individual dose mode $(P=1)$, the code produces Table 1 through 8 with dose reported in mrem. When operating in the population dose mode $(P=0$ or $P>1)$, the code first produces Tables 1 through 8 reporting population dose in man rem. Tables 5 through 8 are then repeated as health effects reported in premature cancer deaths.

\subsection{RADIOLOGICAL DOSE MODEL FOR INTACT FOEL}

The external radiation field dose rates due to a given system will change with time due to radioisotopic decay and buildup, resulting in varying gamma and neutron energy spectra with time. The external dose rates from a given system were calculated using an NOS-modified version of the QAD-P5 code derived from the QAD code originaliy developed by IASL. $(40,41,42)$

QAD is a point kernel code system designed to calculate fast neutron and gamma-ray penetration of various shield configurations. Estimates are made of gamma-ray flux, dose rate, and energy deposition at specified detector locations, accounting for buildup within the source using Capo's parameter definitions and attenuation by shielding materials. The fast neutron dose obtained using a modified Albert-Wetton kernel was found to agree most closely with measure values.

Input data for the code consists of 1) a description of the source distribution and intensity by a number of point isotopic sources, 2) a mathematical representation of the physical geometry with quadratic surface, and 3) a tabulation of attenuation coefficients applicable to the shielding materials, buildup factors, and conversion factors.

In applying the code, equivalent cylindrical geometries are assumed for all systems considered. Thickness and material type were specified for each layer of the system. Gamma photon and neutron production rates and energy spectra as a function of time, derived from the ORIGEN runs described in Chapter 4, are input into successive $Q A D$ runs to determine the external dose rates versus time at specified distances in the axial and radial directions of each system. Measured gamma and neutron dose rates from actual systems were then used to calibrate the calculated values at $t$ ime $t=0$, assuming that the measured values represented fresh fuel. Calculated values for subsequent time periods were scaled accordingly. The results indicated that the calculated and measured values at $t$ ime $t=0$ were in good agreement, being within a factor of two at most. 


\subsection{POPULATION BEALTH EFFECTS MODEL}

The population risk associated with postulated accidents involving systems evaluated in this report is reported in terms of health effects resulting from calculated population doses.

Radiation effects can be classified as either somatic effects that become evident in the exposed individuals, or genetic effects that become evident in the exposed individual's descendents. The late effects of radiation observed in man and animals are induction of cancer, cataracts, impairment of fertility, developmental irregularities of fetus, and shortening of life. There is experimental evidence to show that impairment of fertility and cataract production are threshold events not likely to occur at low dose levels. It is believed that the risk of cancer may be increased by smaller amounts of radiation than are effective for the other types of injuries. Consequently, the somatic injury of primary concern is cancer. The genetic risks of radiation may range from dominant single gene mutants whose effects may be categorically recognized, to subtle genetic contributions to disease conditions that are primarily environmental or nongenetic in origin.

The relationship between radiological doses and resulting health effects, and the model used in calculating such health effects are described in this chapter.

\subsection{Dose Response Models}

The quantitative estimation of the expected health effects resulting from radiation exposure employs data derived from medical, accident, occupational, and warfare related exposure of humans to different radiation sources. These studies have been summarized by the NAS-NRC Committee on the Biological Effects of Ionizing Radiation (BEIR), $(43,44)$ and by the United Nations scientific Comittee on the Effects of Atomic Radiation (UNSCEAR) $(45,46)$ For various reasons, these and other studies have not provided a clear answer to the most pressing and controversial question in radiobiology: what are the health effects of low doses of radiation, delivered in a single exposure or in multiple exposures over time. The 0.S. Department of Health Education and Welfare's (BEW) Interagency Task Force on Ionizing Radiation recently reviewed studies bearing on this question, examined various hypotheses that could explain the data, and concluded that existing knowledge is insufficient to provide an unequivocal answer to the low dose question. (47)

Despite such difficulties, attempts have been made to estimate low dose effects, so that limits may be set on exposures during activities involving radiation. The estimates are premised on two assumptions, which are theoretically sound, but which have been viewed as overestimating the probable effect of low doses: first, that there is no threshold dose level below which radiation exposure 
has no carcinogenic effect; and second, that the incidence of cancer at low doses is directly proportional to the response at high doses. This is the so-called linear dose response model for which the important indicator of population risk is the integral of the population versus dose curve, expressed in man-rem.

Other models have been developed to predict low dose effects. One of these produces a linear quadratic dose response curve, reflecting the hypothesis that cancer incidence is proportionately lower at low doses than at high doses, in part because cells may repair themselves more easily at low doses.

Recently, four studies of populations exposed to very low levels of radiation have been performed. (47) some results of these studies suggest that the linear hypothesis, rather than being conservative may underestimate the $r$ isk of cancer from radiation exposure by a factor of 10 or more. The Hew Task Force review of these four studies concluded that while the studies are suggestive, none of them is sufficiently extensive, complete or free from methodological problems to provide conclusive information at present on the effects of radiation at low doses. (47) However, there are indications that high linear energy transfer (LET) radiation (alpha particle and neutrons) have a higher response per unit dose at low than Low LET radiation ( $x-r a y$, gamma, and beta). (44) If a linear hypothsis, assumed to hold for low LET radiation, is also applied to high LET radiation, then the resulting high LET dose response may indeed be underestimated. This problem has prompted a recommendation to increase the quality factor for alpha particles and neutrons use in dose calculations from 10 to 20 , and other changes to internal dosimetry models.

Bealth effects estimates based on the linear dose response model have been summarized by the BEIR I Committee and UNSCEAR. $(43,44)$ The BEIR and UNSCEAR reports have arrived at comparable risk estimates of the expected mortality from radiation-induced cancer, but the BEIR I report presents these estimates in a form that is more readily applied to the estimation of risk in populations that include the normal age distribution.

The BEIR I report makes estimates of both absolute risk and relative risk of cancer death due to radiation. The absolute risk estimates are based on the difference between the risk in the irradiated population and the risk in the nonirradiated population. The relative risk estimates are based on the ratio on the risk in the irradiated population to the risk in the nonirradiated population. Either type of estimate also involves assumptions about the years at elevated risk following the latent period. For leukemia, the years at elevated risk following a latent period of 2 years was assumed to be 25 years. For all other cancers, either a 30 -year or a duration of life interval of elevated risk was assumed following a latent period of 15 years. This leads to four types of risk estimates for death due to cancer types other than leukemia. Only the two estimates of the latter group which lead to the lowest and the highest predictions of cancer mortality are considered here, the 
the lowest being the absolute risk model with a 30-year plateau, and the highest being the relative risk model with a lifetime plateau. These estimates are presented in columns 3 and 4 of Table 7-1. The corresponding health effects estimates documented by onsCEAR $(46)$ are presented in column 5 of Table 7-1 for comparison.

The health effects resulting from radiation exposure have also been documented by ICRP in ICRP Report $26 .(48)$ The ICRP $r$ isk estimates, presented in column 6 of Table 7-1, were derived from the BEIR I report, and essentially correspond to the low end BEIR report risk estimates based on the absolute risk model with a 30-year plateau of elevated risk following the latent period. Earlier estimates of the health effects resulting from radiation exposure have also been documented by the National Council on Radiation Protection and Measurements (NCRP). (49)

The EPA has adopted the policy of assuming a linear relationship between the population exposure and its biological effect. $(50,51)$ Information contained in References 43 and 44 formed the basis for this policy decision. In arriving at health effect estimators, EPA has adopted those based mainly on the average of the two $r$ isk models considered in the BEIR I report, corresponding to models for relative and absolute risk, respectively. The resulting EPA risk estimators are presented in column 7 of Table 7-1.

The linear dose response model has been adopted by the International Commission on Radiation Protection (ICRP), the O.S. Enviconmental Protection Agency (EPA), and the O.S. Nuclear Regulatory Commission (NRC) for planning purposes. Models adopted in the recent official BEIR III report are discussed in detail in Section 6.2. However, it was not possible to incorporate the BEIR III results into the present model at this time. The linear dose response model is considered conservative, especially for low dose levels, when compared to the linear-quadratic model. For planning purposes the linear dose response model has been adopted in this report. Such an approach is consistent with the current position of regulatory agencies such as EPA, as well as the ICRP.

The population doses calculated at the end of each time step of the model are transformed into health effects using the following relationship:

$$
B_{i}=\sum_{j} D_{i j} h_{j}
$$

where

$\mathbf{H}_{i}=$ cancer deaths as a result of radiological exposure
during time step $i$ 
Table 7-1. Alternative Health Befects Risk Bstimator for Radiation Exposure

\section{Health Befects Risk Bstimators, effects/ren-yra}

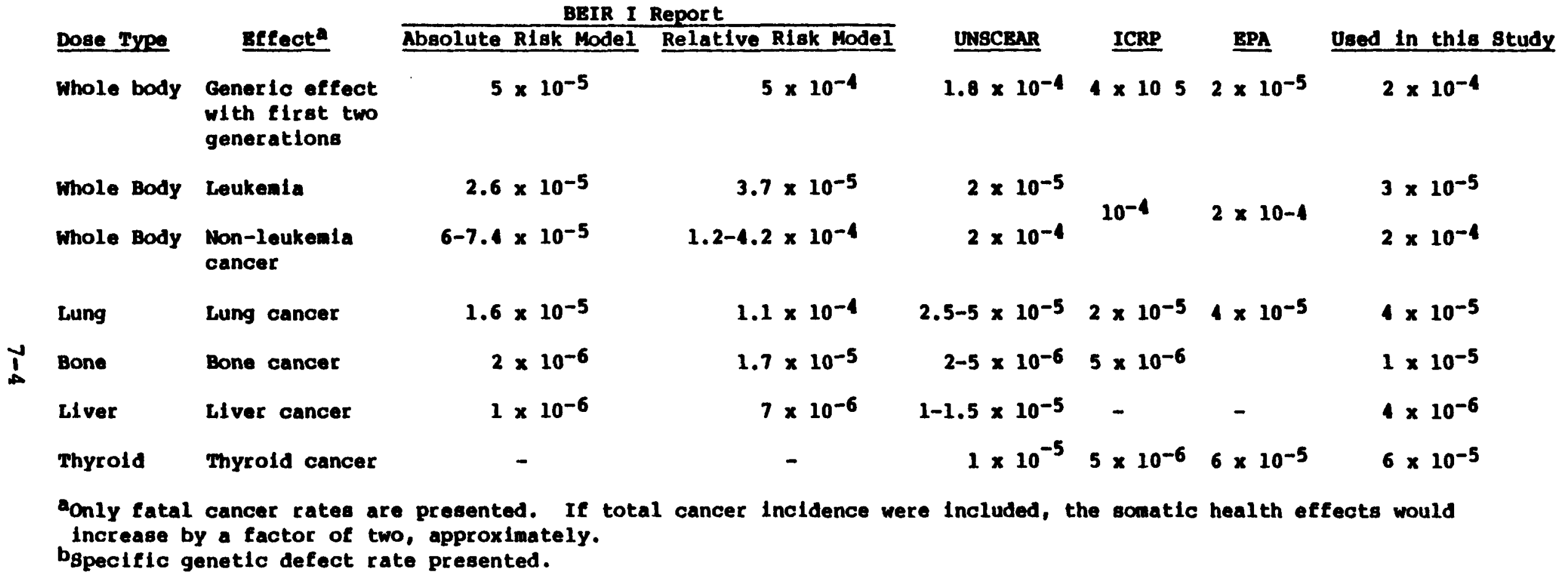




$$
\begin{aligned}
D_{i j} & =\text { population dose to organ } j \text { during time } i \text {, person-rem } \\
h_{j} & =\begin{array}{l}
\text { health effects estimator for organ } j \text {, cancer deaths/ } \\
\text { person-rem }
\end{array}
\end{aligned}
$$

\subsection{BEIR III REPORT SUMMARY}

The BEIR committee has recently published a detailed review of available data on radiation-induced health effects entitled The Effects on Populations of Exposure to Low Levels of Ionizing Radiation (known as BEIR III). (44) The BEIR III uses a variety of methods and data to quantify the health impacts of low levels of radiation. The appendix provides quantitative estimates of the health risks associated with low levels of ionizing radiation based on the BEIR III findings. This appendix does not deal with the immediate effects of high levels of radiation often referred to as "acute radiation syndrome."

The BEIR III identifies three categories of radiation-induced human health effects: (1) cancer, (2) genetic disorders, and (3) somatic effects other than cancer. The committee believes that cancer induction is the most important effect of low-dose radiation. In this context, "Iow dose" refers to chronic doses of up to a few rads per person per year or acute exposures of up to 25 rads. Natural background radiation ranges from 0.1 to 0.2 rads per person per year. Genetic effects of low-level radiation have been well documented and are addressed in detail in BEIR III.

The BEIR III concludes that low-dose exposure of human populations does not increase the risk of somatic effects other than cancer and developmental changes in unborn children. The report also indicates that developmental changes in unborn children are probably not caused by radiation at or below natural background levels. For these reasons, only cancer and genetic disorders are considered in this analysis.

\subsubsection{CANCER RISK ESTIMATES}

The BEIR III uses a variety of data, mathematical models, projection models, and exposure situations to quantify the risks of radiation-induced cancers. The final estimates of risk can vary widely based on the selected data and models. Furthermore, there are many uncertainties associated with the data utilized in the BEIR III analysis that preclude strict quantitative interpretation of the BEIR III risk estimates.

Radiation dose is measured in a number of units. The "rad" is the basic unit of absorbed dose in terms of energy per unit mass. One rad equals 0.01 joules/kilogram. The rad, however, does not include a consideration of the relative biological damage caused by different types of radiation. This relative damage is considered in the unit "rem" which is referred to as the dose equivalent. The rem is simply the rad multiplied by a quality factor 
to account for the relative biological damage caused by the type of radiation under consideration. The quality factor is determined from the "linear energy transfer" of the type of radiation.

Ionizing radiation can generally be characterized as either low or high LET. LOW LET radiation includes gamma and $x$-radiation and electrons while high LET radiation includes alpha particles and neutrons. IET is a measure of the rate at which the energy of the radiation is transferred to human tissue as it passes through the tissue. Some gamma radiation (low LET) can pass right through the human body without depositing its energy. The energy of high LET radiation, like alpha particles, is generally all deposited in the tissue in which it is emitted.

The BEIR III report presents detailed quantitative analyses for the health risk from low LET radiation only. The health risks from high LET radiation are discussed in less detail. There is considerable controversy over biological effects of low doses of high LET radiation. Estimates of health risk from both high and low LET radiation are presented here. They are based on the best available information from the BEIR III report. It is possible that future investigations into the effects of high LET radiation could greatly alter these estimates.

Cancer data from the Japanese survivors of atomic bombs are used in most of the analyses in the BEIR III report. Individual dose rates to these individuals were very high compared to the low dose rates described above. A major question addressed by BEIR III is how to extrapolate the cancer risks observed at the relatively high dose rates down to the lower dose rates. BEIR III has adopted a parametric family of functions to accomplish this extrapolation. These functions or models are the (1) Iinear, (2) linear quadratic, and (3) quadratic. The linear model has been the model endorsed in previous BEIR committee reports and is the model that results in the highest estimates of risk. This model is also endorsed in the BEIR III report for quantitating the genetic risks of radiation. The linear-quadratic model has been endorsed by BEIR III for estimating cancer risk from low LET radiation. The endorsement of this model is a significant change from the previous recomendations of the BEIR committee. The risk estimates using this model are somewhat lower than those of the linear model. The quadratic model results in the lowest risk estimates.

The BEIR III report does not make a firm recommendation on the most applicable model for use in estimating the risk from high LET radiation. However, based on statements made in the report, it is believed that the linear model would provide the best available estimate of cancer risk from this type of radiation.

The rad (absorbed dose) is used for the health risk estimates for low LET radiation in BEIR III. If a quality factor of 1 is assumed for the low LET radiation, the estimates also apply on a per rem basis. Since doses are generally calculated in 
units of rem, the health risk estimates presented here are on a per rem basis.

One characteristic of cancer is that it takes a long time to develop, referred to as an expression time or latent period. Leukemia has a characteristically short expression time (less than 25 years), while other cancers can have expression times up to the life span of an individual. Because only about 30 years of cancer data have been collected on the survivors of the atomic bombs, the data do not account for all of the cancers (other than leukemia) that might develop because of the bomb's radiation. Two projection models have been developed to account for these future cancer deaths: (I) the absolute risk projection model assumes that the cancer rate ( $r$ isk per year) observed since the atomic bomb blasts will continue throughout the life span of those exposed; (2) the relative risk model assumes the excess radiation-induced $r$ isk is proportional to the natural incidence of cancer with age. The relative risk model results in cancerrisk estimates greater than those predicted by the absolute model. However, BEIR III states that the absolute model is generally more applicable to most forms of cancer. However, a controversy exists over which projection model should be used. Estimates using both projection models as well as the arithmetic average of the two projection models are presented here.

The BEIR III states that the age and the sex of individuals exposed are important factors in determining the risk of cancer from the radiation exposure. All BEIR III risk estimates include values for both males and females. For this analysis, the arithmetic average of the risk estimates for the two sexes has been used. (The slightly higher number of females than males does not warrant a weighted average by sex.) The BEIR III presents annual age-specific cancer risk coefficients. These coefficients are used in computerized life-table calculations to determine the number of excess cancer deaths per million persons for various exposure situations. Estimates are made for a single exposure of $10 \mathrm{rads}$ and chronic exposure to $1 \mathrm{rad} / \mathrm{year}$ over a Iifetime and over three other age brackets. The life-table estimates of total cancer risk for these two exposure situations include an assumed age distribution for the United states and also assumes that the individual dose rate is the same for all ages. This analysis has used the life-table estimates.

Because not all cancers are fatal, BEIR III presents cancer risk as both cancer fatalities and cancer incidence. About 50 percent of all cancers are fatal for females, while 65 percent are fatal for males. It is difficult to judge the societal cost of cancer death, although it does provide a common base on which the risks of various human activities can be compared. Cancer incidence is more difficult to judge because of the varying degrees of ill bealth and of treatment costs. Some members of the BEIR III committee feel that cancer incidence is a more complete index of risk. Eowever, the BEIR III also states that the ". . . uncertainties surrounding the data bases for incidence are greater than those 
for mortality." Consequently, this analysis evaluates cancer mortality only.

The cancer-risk estimates used in this analysis have been developed from the data in Tables $V-14$ through $V-21$ of the BEIR III report. The life-table estimates of excess fatal cancer cases per million persons from a single exposure to 10 rads and from continuous lifetime exposure to 1 rad per year were utilized. For both the linear and linear-quadratic models, these values can then be applied directly to population doses if individual chronic dose rates are less than 12 rads per person per year and if acute exposures are less than 25 rads. At higher doses, the quadratic component of the Iinear-quadratic model becomes significant, and individual dose rates must be considered in the evaluation of cancer $r$ isk. This relationship is depicted in Figure 7-1. BEIR III defines the risk of fatal cancer, other than leukemia and bone cancer, using the linear quadratic model as:

$$
\text { Excess Risk }=1.397\left(D+0.008614 D^{2}\right)
$$

where $D$ is in units of rads. This formula is utilized in the life-table computations to obtain the number of excess fatal cancers per million persons from a single exposure of 10 rads or continuous exposure to $1 \mathrm{rad} /$ year over a 1 ifetime $(67$ years for males, 75 years for females). To apply these estimates to other dose rates, the calculated number of cancers per million persons can be divided by the total exposure to yield risk factors in units of fatal cancers/106 man-rad. Solving equation 7-2 for the $1 \mathrm{rad} /$ year exposure situation and applying the results linearly is equivalent to converting equation 7-2 to:

$$
\text { Excess Risk }=1.409 \cdot D
$$

As can be seen in Figure 7-1, the results of equations 1 and 2 are very similar at low doses. The difference in the results of the two equations is less than 10 percent at a dose of 12 rads. A similar computation can be performed for the 10 rad acute exposure situation. The computation indicates that a 10 percent difference is reached at 25 rads. This computation also shows that the Iinear application of the Iinear quadratic model for acute exposures of 1 rad or less results in a 8 percent overestimate of $r$ isk. These inaccuracies associated with the linear application of the linear-quadratic model are deemed acceptable.

Risk estimates for total cancers other than leukemia and bone cancer resulting from total body exposure were taken from Tables $V-19$ and $V-20$ of BEIR III. Leukemia and bone cancer risk estimates were taken from Tables $v-17$ and $v-18$. All of these estimates are based on the Life Span study Mortality Data for Japanese bomb survivors. These risk estimates are presented in Table 7-2. 


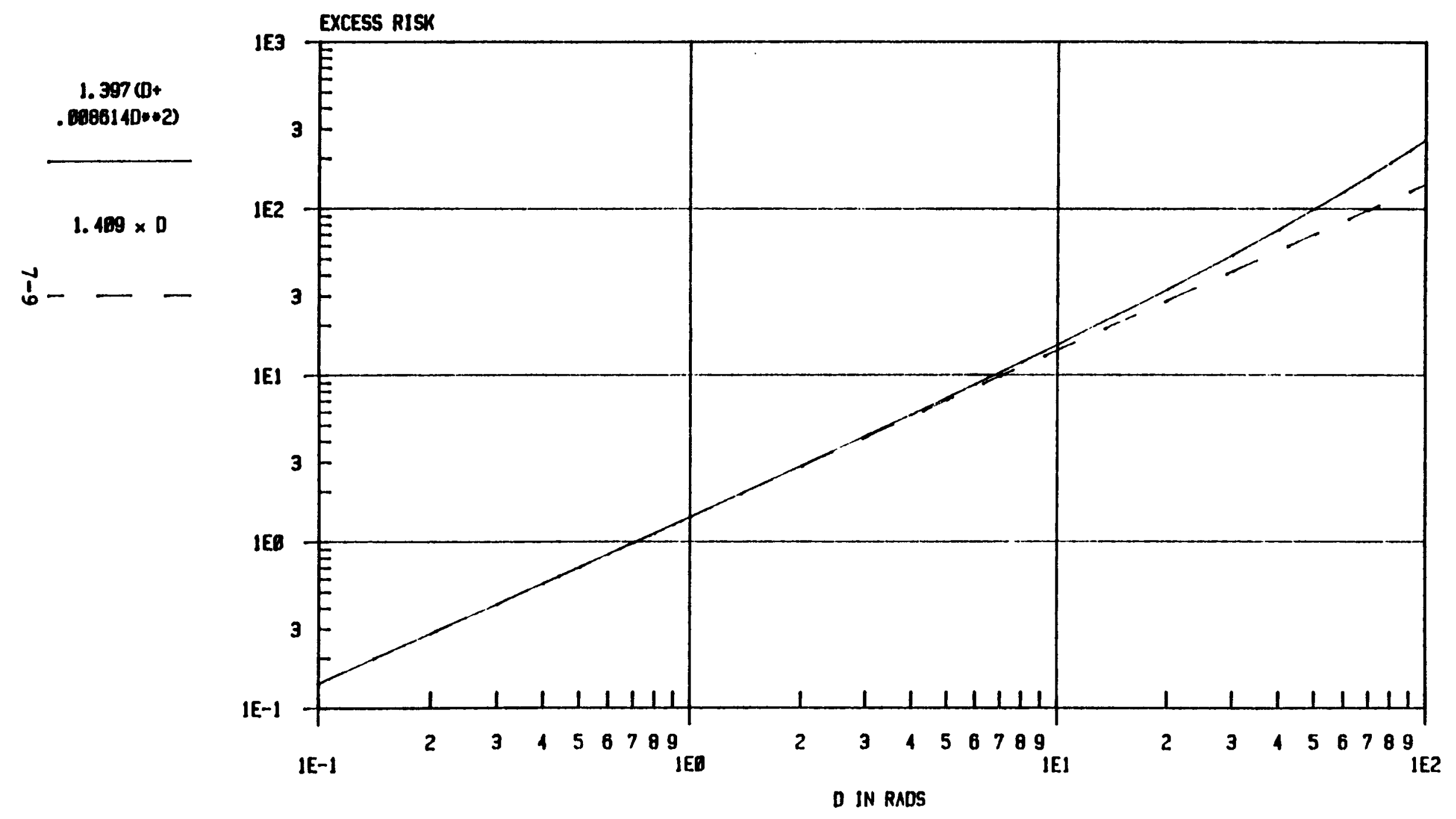

Figure 7-1. Comparison of computational methods for BEIR III risk estimates. 
Table 7-2. BEIR III Cancer Risk Betinates - Fatal Cancers Per Million Man-Rem of Total Body Exposurea

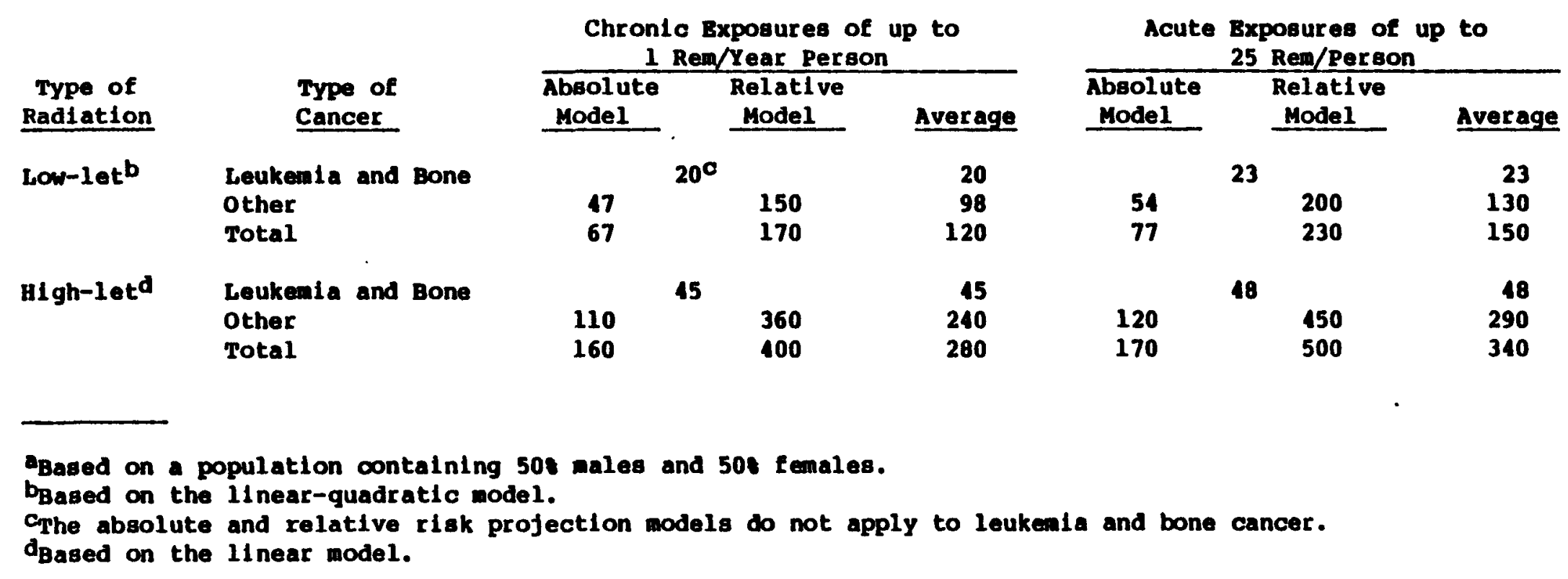


Comparison of these data indicates the acute exposure results in slightly higher cancer risk per rem than chronic exposure. The cancer risk per rem from high LET radiation using the linear model is more than a factor of two higher than the cancer risk/ rem of low LET radiation (Iinear-quadratic model). Since these estimates are in units of rem, the high LET doses with which the risk estimates are combined already include a quality factor of 10 or 20 . The total difference in the cancer causing potential of the two types of radiation is a factor of between 20 and 40 .

The BEIR III does not present life-table estimates of cancer risk on a cancer-site- (organ-) specific basis. Cancer-risk coefficients for specific sites are developed in BEIR III from a variety of data sources and are presented in BEIR III Table V-14. Site-specific risk estimates for this analysis were calculated with the following formula:

$$
H_{i}=\frac{a_{i} R_{i}}{\sum\left(a_{i} x R_{i}\right)} H_{t}
$$

where:

$$
\begin{aligned}
B_{i}= & \text { the organ-specific health risk estimates for organ } i \\
& \text { in cancer deaths per } 106 \text { man-rem; } \\
a_{i}= & \text { the organ-specific cancer incidence coefficients from } \\
& \text { BEIR III Table } v-14 \text { in } 1 / 106 \text { rad-years; } \\
R_{i}= & \text { the mortality per incidence ratio for each cancer site } \\
& \text { from BEIR III Table v-15; } \\
B_{t}= & \text { an appropriate life-table risk estimate for all cancers } \\
& \text { other than leukemia and bone cancer in cancer deaths } \\
& \text { per } 106 \text { man-rem. }
\end{aligned}
$$

These calculations are shown in Table 7-3.

Most dose assessments include the calculation of doses to the total body or the total body and some combination of other organs. In exposure situations which are limited to external exposures, doses to the total body only are calculated.

In these cases, the total body doses are multiplied by the total cancer risk estimates in Table 7-2 to estimate the number of cancer fatalities. In this case, the total number of cancer fatalities is estimated by:

$$
C F=D_{\mathrm{T}} \cdot \mathrm{B} \cdot \mathrm{E}_{\mathrm{T}}
$$

where $D_{T, B}$ is the total body population dose and $B_{T}$ is the total cancer risk estimator from Table 7-2. 
Table 7-3. Excese Cancer Incldence and Mortallty Per M1111on Persone Per Year Per Rad by Cancer site (Organ)

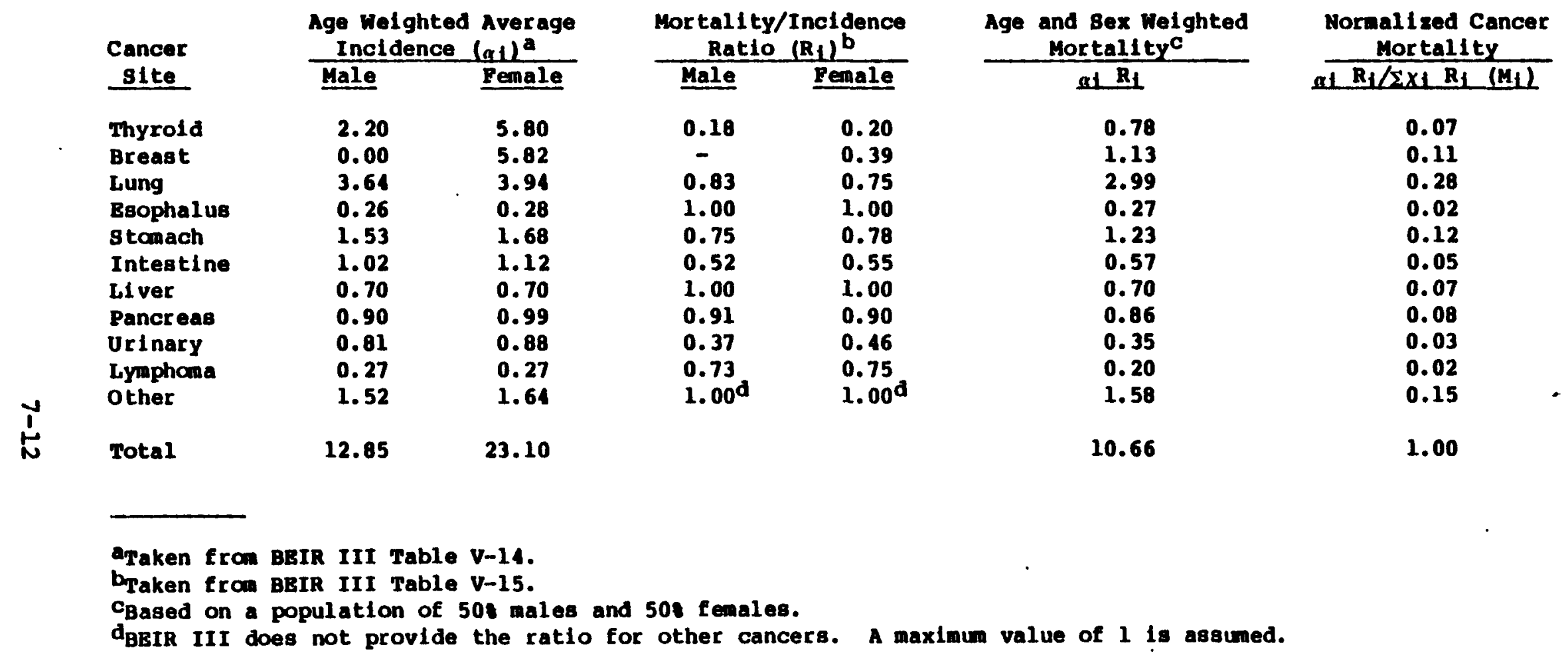


In other instances, both total body and organ doses are calculated. When the total body doses are calculated from external exposure only and organ doses from internal exposure only, the total cancer fatalities are estimated by:

$$
C F=D_{T . B .} H_{T}+\sum_{I}^{n} D_{i} M_{i} B_{0}+D_{\text {Bone }} B_{L}
$$

where $n$ is the number of organs for which doses have been calculated, $D_{i}$ is the population dose organ $i, M_{i}$ is the normalized cancer mortality for organ $i$ (Table 7-3), $\mathbb{H}_{0}$ is the cancer $r$ isk estimator for cancers other than leukemia and bone cancer (Table 7-2), DBone is the population bone dose, and $\mathrm{B}_{\mathrm{L}}$ is the leukemia and bone cancer estimator (Table 7-2).

In most instances, the calculated total body and organ-specific doses account for all dose pathways including both internal and external exposure. In this case, the total number of cancer fatalities is estimated by:

$$
C F=\sum_{I}^{n} D_{i} M_{i} B_{O}+\sum_{n+I}^{k} M_{i} \quad D_{T B} H_{O}+D_{B O n e} B_{L}
$$

where $k$ is the total number of organs listed in Table 2 including the "other" category. In this case, the total body dose is being used as the dose to all organs for which doses have not been calculated.

Genetic Risk Estimates.

Genetic disorder risk estimates based on BEIR III are more straightforward than cancer risk. BEIR III Table IV-2 estimates that between 60 and 1100 genetic disorders (sum of autosomal dominant and irregularly inherited) will occur from a gonadal dose of $1 \mathrm{rem} / g$ eneration/million liveborn offspring in an equilibrium population. An equilibrium population is defined as one in which there is one liveborn offspring per person per generation (2 children per family). This is equivalent to saying that from 60 to 1100 disorders will occur man-rem gonadal dose. These two values were used in this analysis as the range of genetic disorders with their geometric mean of 257 as the "probable" risk value. These estimates have not changed significantly from the previous BEIR committee results. Dose to the gonads is generally used in estimating genetic risks, however, if gonadal doses are not available, total body doses may be used. 


\subsection{UPDATE ON INTERNAL DOSE MODEL}

Some comments are in order regarding the current validity of the internal dose rate conversion factors for Pu-238 developed in OSM Vol. 2. The recently gublished ICRP 30 presents an updated internal dosimetry model. $\{52)^{2}$ The basic lung dynamic model remains the same, except that the dose due to cross irradiation of one organ by gamma photons from another organ is included in the analysis. This will not affect calculated doses for Pu-238 because the alpha radiation doses completely. dominate the gamma photon radiation doses anyway. However, there was a change in the value of $f_{1}$ for Pu-238, the fraction of activity in the small intestine that is transferred to the blood, which was increased Erom $10^{-6}$ to $10^{-5}$ for $Y$ solubility class compounds. This will not affect the inhalation dose rate conversion factors, because the fraction of inhaled activity cleared to the blood through the small intestines can be considered negligible, amounting to $10^{-5}$ to $4.3 \times 10^{-5}$ of the inhaled activity cleared from the respiratory system directly to the blood, depending on the region cleared. However, straight ingestion doses, not considered in the analysis, will increase in importance by an order of magnitude, making the ingestion pathway more important than previously.

Another important change is how doses are calculated. In Reference 53 (ICRP 2) a quality factor of 10 was used for alpha radiation, along with a relative damage factor of 5 and a bone mass of $7000 \mathrm{~g}$ yielding a dose calculation multiplier of $7.14 \times 10^{-3}$. ICRP 30 now recommends a quality factor of 20 for the bone, but no mention is made of the use of damage factor. Instead, the bone is divided into two parts, the bone surface and red marrow with masses of 120 and $1500 \mathrm{~g}$, respectively. All isotopes of plutonium are assumed to be uniformly distributed over bone surfaces at all times following their deposition in the skeleton. A new term called the absorbed fraction is defined, which is the fraction of energy emitted as a given radiation type in a specified source tissue which is absorbed in a specified target tissue. When this is taken into account, the new multipliegs for Pu-238 become $4.17 \times 10^{-2}$ for bone surfaces and $1.33 \times 10^{-3}$ for red marrow per unit of bone activity burden.

Aside from changes in the internal dose model described above, results will vary considerably depending on what particle size distribution is selected for analysis. This aspect of internal dosimetry has not changed. Caution should especially be exercised before ignoring particle sizes above 4 microns. Although particle sizes below 4 microns have large dose rate conversion factors on a per unit of activity inhaled basis, particle sizes up to 100 microns or larger can be deposited in the respiratory region, primarily in the nasopharyngeal region. The small dose rate conversion factors of these large particles is balanced by their large mass fraction, and hence activity, that is deposited in the nasopharyngeal region. This effect can be seen when a typical particle spectrum for fractured puO $_{2}$, based on 
LASL impact stugijes and presented in OSM, Vol. 2 Table 2.1-3, is considered. (2) Using the OSM doses results as an example, inhalation dose rate conversion factors as a function of particle size for a flat particle size spectrum and the typical particle size spectrum cited above are presented in Figures 8-1 and 8-2, respectively, for the bone. $(54)$ There is no difference in the lung dose due to this effect, consistent with the past assumption that particles above 4 microns can be ignored when lung doses only are of concern. However, the differences in doses to the bone are tremendous, with particles above $4 \mathrm{microns}$ accounting for most of the bone doses. This is due to the large amount of activity deposited in the nasopharyngeal region that is subsequently cleared to the blood.

ICRP 30 handles the problem of particle size distributions by presenting respiratory system inhalation deposition fractions, not in terms of given actual particle size, but in terms of the activity median aerodynamic diameter (AMAD) of log normal particle size distributions with AMAD's in the range of 0.2 to 10 microns and geometric standard deviations of less than 4.5 microns.

Caution should be exercised at this point when considering the deposition and transfer of large particles in the nasopharyngeal region. References 42 and 45 indicate that $10^{-2}$ of the activity deposited in the nasopharyngeal region is transferred to the blood with a clearance half-time of 0.01 days. Nus has contended in the past that such a large rapid transfer of large particles directly to the blood is implausible, and that recommendations are made by NuS to restrict the fraction cleared to a value to that for the small intestine $\left(10^{-6}\right.$. previously and now $10^{-5}$ ). The two approaches are reflected in the curves in Figures 8-1 and $8-2$. This problem is further discussed in Reference 53. 


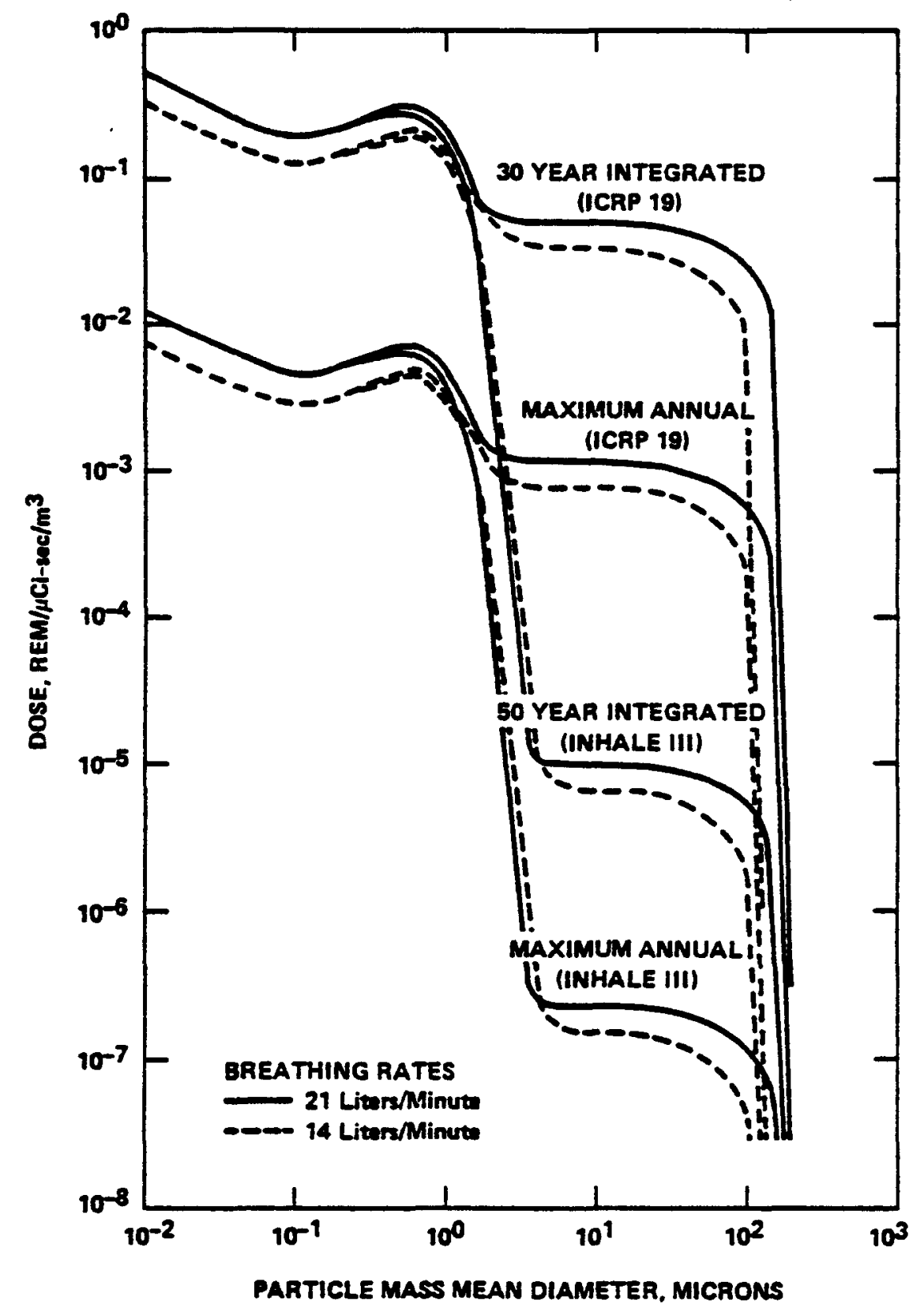

Figure 8-1. Pu-238 inhalation doses (bone) using INHALE III and ICRP 19 region fraction for $\mu$, with fiat particle size distribution. 


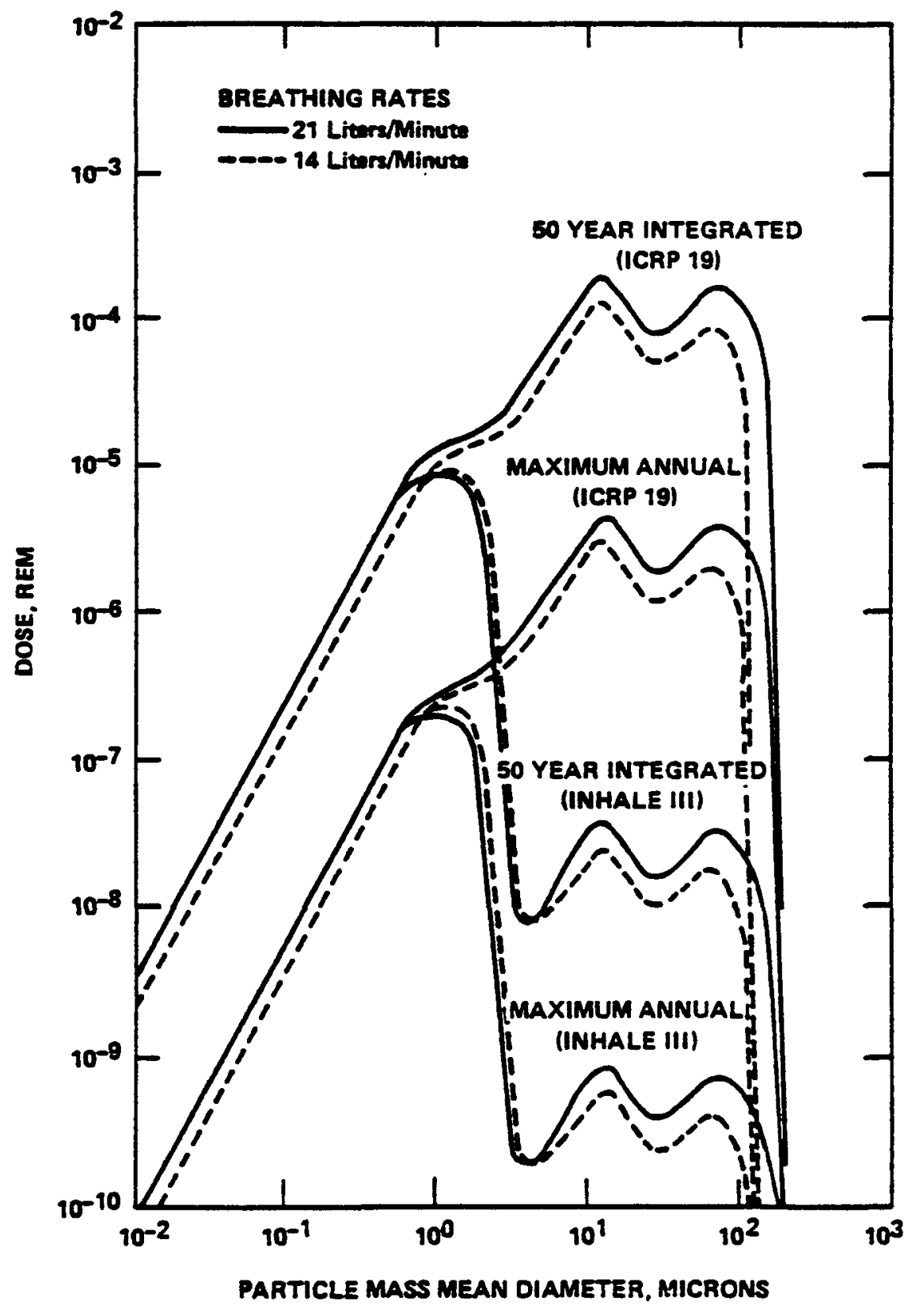

Figure 8-2. PU-238 inhalation doses (bone) using INHALE III and ICRP 19 region fraction for $\mu$, with $1 \mu \mathrm{Ci}$-sec/ $/ \mathrm{m}^{3}$ or entire PPO particle size distribution (270 ft/sec impact). 


\subsection{REFERENCES}

1. Bartram, B. W. and R. W. Fell, Plutonium Long Term Risk Model, Prepared for U.S. Department of Energy by NuS Corporation, NUS-3608 (June 30, 1980).

2. NUS Corporation, Overall Safety Manual, Prepared for 0.S. Atomic Energy Commission (June 1975).

3. Remeny, J. G., and J. I. Snell, Finite Markov Chains, D. Van Nostrand Company, Princeton, New Jersey (1960).

4. Eeldman, C., and M. J. Malmberg, Plutonium Monitoring in the Environment, NUS Corporation Report 3033 (September 1977).

5. Hakonson, T. E., "Environmental Pathways of Plutonium into Terrestrial Plants and Animals," Health Physics, 29, 583 (1975).

6. Mullen, A. A., and R. E. Mosley, Availability, Uptake and Translocation of Plutonium Within Biological Systems: A Review of Significant Data, PB-252 179 (April 1976).

7. Cohen, B. L., "Hazards from Plutonium Toxicity," Health Physics, 32, 359 (1977).

8. Comar, C. I., Plutonium: Facts and Influences Summary Report, PB-260 720 (August 1976).

9. Tamura, T., Physical and Chemical Characteristics of Plutonium in Existing Contaminated Solids and Sediments, IAEASM-199/52, International Atomic Energy Agency, Vienna (1975).

10. Hakonson, T. E., et al.. Accumulation and Transport of Soil Plutonium in Liquid Waste Discharge Areas at Los Alamos, IAEA-SMI99799, International Atomic Energy Agency, Vienna (1975).

11. Bernhardt, D. E., Evaluation of Sample Collection and Analysis Techniques for Environmental Plutonium, PB-253960 (April 1976).

12. Little, C. A., Plutonium in a Grassland Ecosystem, dissertation, Colorado State University, Fort Collins, Colorado, CoO-1156-83 (1976).

13. Fleincher, R. L., "On the Dissolution of Respirable $\mathrm{PuO}_{2}$ Particles," Eealth Physics, 29, 69 (1975).

14. Romney, E. M., et al., Eealth Physics, 19, 487 (1970).

15. Gaury, J. C., and A. Fraizier, "Influence of Trophic Level and Calcification on the Uptake of Plutonium Observed In situ, in Marine Organisms," Health Physics, 32, 21 (1977). 
16. Aarkrog, A., "Envirommental Behavior of Plutonium Accidentally Released at Thule, Greenland," Health Physics, 32 , 271 (1977).

17. Schell, W. R., and R.L. Watters, "Plutonium in Aqueous Systems," Eealth Physics, 29, 589 (1975).

18. Oak Ridge National Laboratory, ORIGIN Isotope Generation and Depletion Code, Matrix Exponential Method, CCC-217 (November 8, 1975).

19. TRW, Safety Analysis Report for Transit, TRW (A) -111464291 (April 24, 1970).

20. Martin Marietta, Nuclear Division, SNAP 29 Preliminary Safety Analysis Report, MND-2062-3314-1 (June 1968).

21. Buge, K., P. Brown, G. Arnason, S. Lazarick, and M. Levitz, A Computer Program for the Fall and Dispersion of Particles in the Atmosphere, Prepared for Sandia Corporation by Travelers Research Corporation, SC-CR-67-2530 (1967).

22. U.S. Nuclear Regulatory Commission, Calculation of Annual Doses to Man from Routine Releases of Reactor Effluents for the Purpose of Evaluating Compliance with 10CFR50, OSNRC Regulatory Guide 1.109, Rev. I (October 1977).

23. National Academy of Sciences - National Research Council, Committee on the Biological Effects of Ionizing Radiation (BEIR I), The Effects on Populations of Exposure to Low Levels of Ionizing Radiation (1972).

24. National Academy of Sciences - National Research Council, Committee on the Biological Effects of Ionizing Radiation (BEIR III), The Effects on Populations of Exposures to Low Levels of Ionizing Radiation (1980).

25. Bateman, H., "The Solution of a System of Differential Equations Occurring in the Theory of Radioactive Transformations," Proc. Cambridge Phil. Soc., 15, 423-427 (1910).

25. Perez, I. and J. Coleman, A Comprehensive Dose Model and Computer Code for Inhaled Radioactive Particles, NuS Corporation Report 596 (June 1969).

26. National Aeronautics and Space Administration, Orbital Plight Handbook - Part I, Basic Techniques and Data, Prepared by Marshall Space Flight Center (1963).

27. Feldman, C. and M. Malmberg, Plutonium Monitoring in the Environment, Nus Corporation Report 3033 (September 1977).

28. Comar, B. Plutonium: Facts and Influences Summary Report, PB-260 720 (August 1976). 
29. Sagendorf, J. F., Xoodoo Program for Evaluating Atmospheric Dispersion from Nuclear Power Stations, National Oceanic and Atmospheric Administration, NOAA-TM-ERL-ARL-42 (May 1974).

30. D.S. Nuclear Regulatory Commission, Methods for Estimating Atmospheric Transport and Dispersion of Gaseous Effluents in Routine Releases from Light-Water-Cooled Reactors, USNRC Regulatory Guide 1.111 (1977).

31. Turner, D. B., Norkbook of Atmospheric Dispersion Estimates, U.S. Environmental Protection Agency, AP-26 (1970).

32. Briggs, G. A., Plume Rise, AEC Critical Review Series, TID25075 (1969).

33. Chandler, M. M. Final Results of a Worldwide Meteorological Study of Ground Level Atmospheric Dispersion Parameters, wuS Corporation Report 1157 (March 1974).

34. Bartram, B. W. and D. W. Pyatt, Safety Assessment for Space Reactors, Prepared for U.S. Department of Energy by NUS Corporation, Nus-3442 (December 1979).

35. O.S. Nuclear Regulatory Commission, Generic Environmental Impact Statement on Uranium Milling (Draft), NUREG-05II (April 1979).

36. Edington, D., J. Alberto, M. Wahlgren, J. Kartunen, and C. Reeve, Plutonium and Americium in Lake Michigan Sediments, IAEA-SM-199/47, International Atomic Energy Agency, Vienna (1975).

37. U.S. Nuclear Regulatory Commission, Users Guide to GASPAR Code, NUREG-0597 (June 1980).

38. Thompson, S. E., C. A. Burton, D. J. Quinn, Y. L. Ng, Concen tration Eactors of Chemical Elements in Edible Aquatic drganisms, URIL-50564, Rev. I (October 1972).

39. Rocher, D. C., "Dose Rate Conversion Factors for External Exposure to Photon and Electron Radiation Erom Radionuclides Occurring in Routine Releases from Nuclear Fuel Cycle Facilities," Health Physics, Vol. 38, Number 4, P. 543 (April $1980)$.

40. Malenfant, R. E., QAD: A Series of Point Kernel General Purpose Shielding Programs, Los Alamos Scientific Laboratory, LA-3573 (April 1967).

41. Oak Ridge National Laboratory, $Q A D$, Point Rernel General Purpose Shielding Codes: OAD IV, OAD P-5, OAD-B, OAD-V, QAD-INT, OAD-BD, OAD-5R, OAD-BR, and OAD-P5A, ORNL-4181. 
42. Huang, R., QAD-SO: NUS Modified QAD-P5, NUS Corporation, NUS-TM-EC-16 (November 1973).

43. National Academy of Sciences - National Research Council, Committee on the Biological Effects of Ionizing Radiation (BEIR I), The Effects on Populations of Exposure to Low Levels of Ionizing Radiation (1972).

44. National Academy of Sciences - National Research Council, Committee on the Biological Effects of Ionizing Radiation (BEIR III), The Effects on Populations of Exposures to Low Levels of Ionizing Radiation (1980).

45. United Nations Scientific Committee on the Effects of Atomic Radiation (UNSCEAR), Ionizing Radiation: Levels and Effects, Volumes I and II (1972).

46. United Nations Scientific Committee on the Effects of Atomic Radiation (ONSCEAR), Sources and Effects of Ionizing Radiation (1977).

47. D.S. Department of Health, Education, and Welfare, Summary of Work Group Reports of the Interagency Task Force on Ionizing Radiation (Draft) (February 27, 1979).

48. International Commission on Radiation Protection, Recommendations of the International Commission on Radiological Protection, ICRP Publication 26 (January 17, 1979).

49. National Council on Radiation Protection and Measurements, Basic Radiation Protection Criteria, NCRP Report 39 (January 15, 1971).

50. U.S. Environmental Protection Agency, "EPA Policy Statement on Relationship Between Radiation Dose and Effect," Federal Register, Vol. 41 (March 3, 1975).

51. O.S. Environmental Protection Agency, Radiological Quality of the Environment in the United States, EPA 520/1-77-009 (September 1977).

52. International Commission on Radiological Protection, Limits for Intakes of Radionuclides by Workers, ICRP 30, Part A, Pergamon Press, New York (1979).

53. International Commission on Radiological Protection, ICRP 2, Report of Committee II on Permissible Dose for Internal Radiation, Pergamon Press, New York (1959).

54. Bartram, B. W., "Pu-238 Inhalation Doses Due to Large Particles," NoS Corporation Memorandum ESD-76-465 (AQ) (JulY $16,1976)$. 


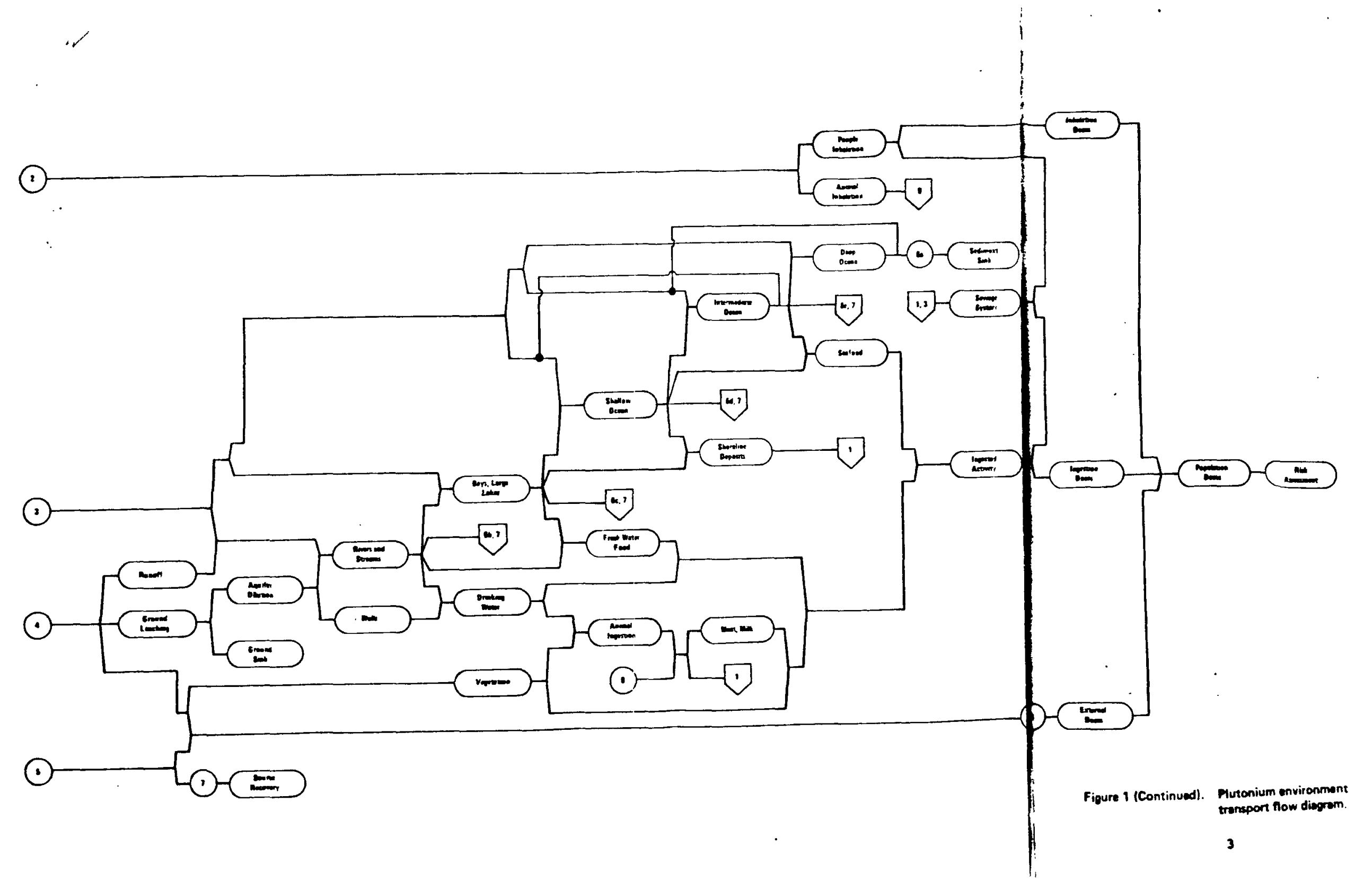




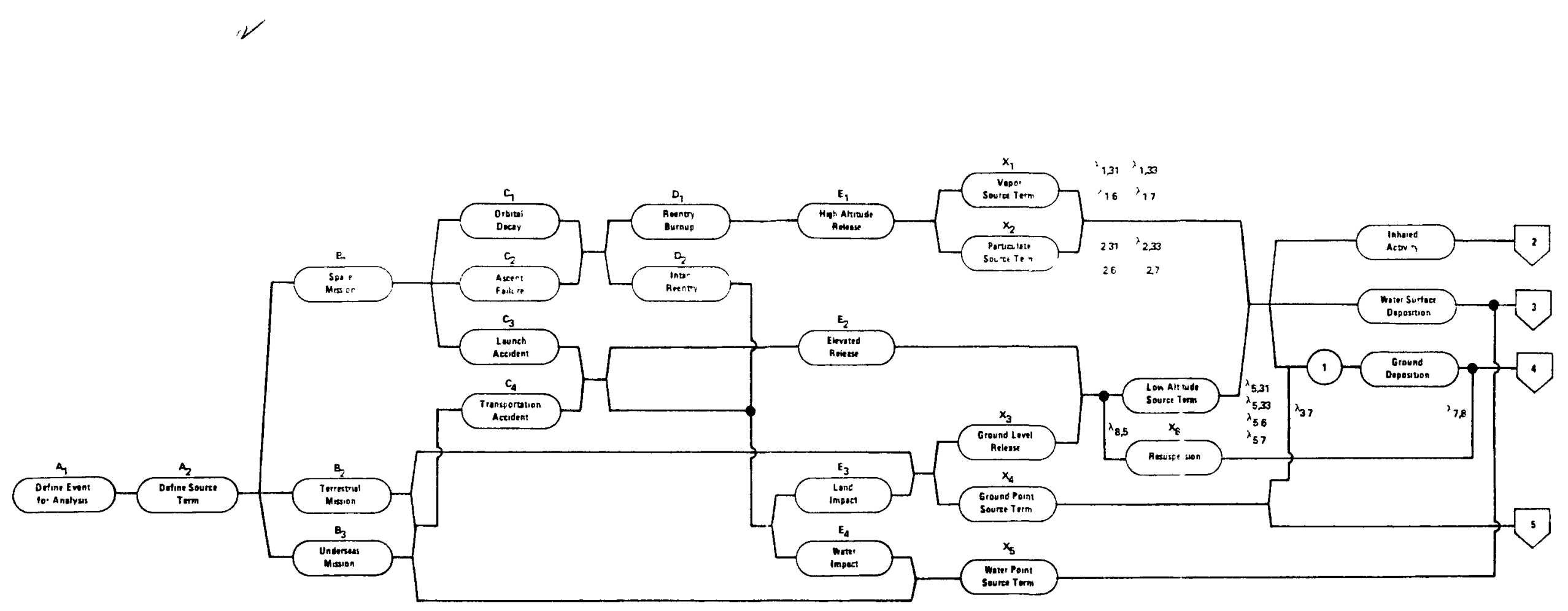




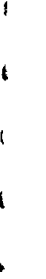

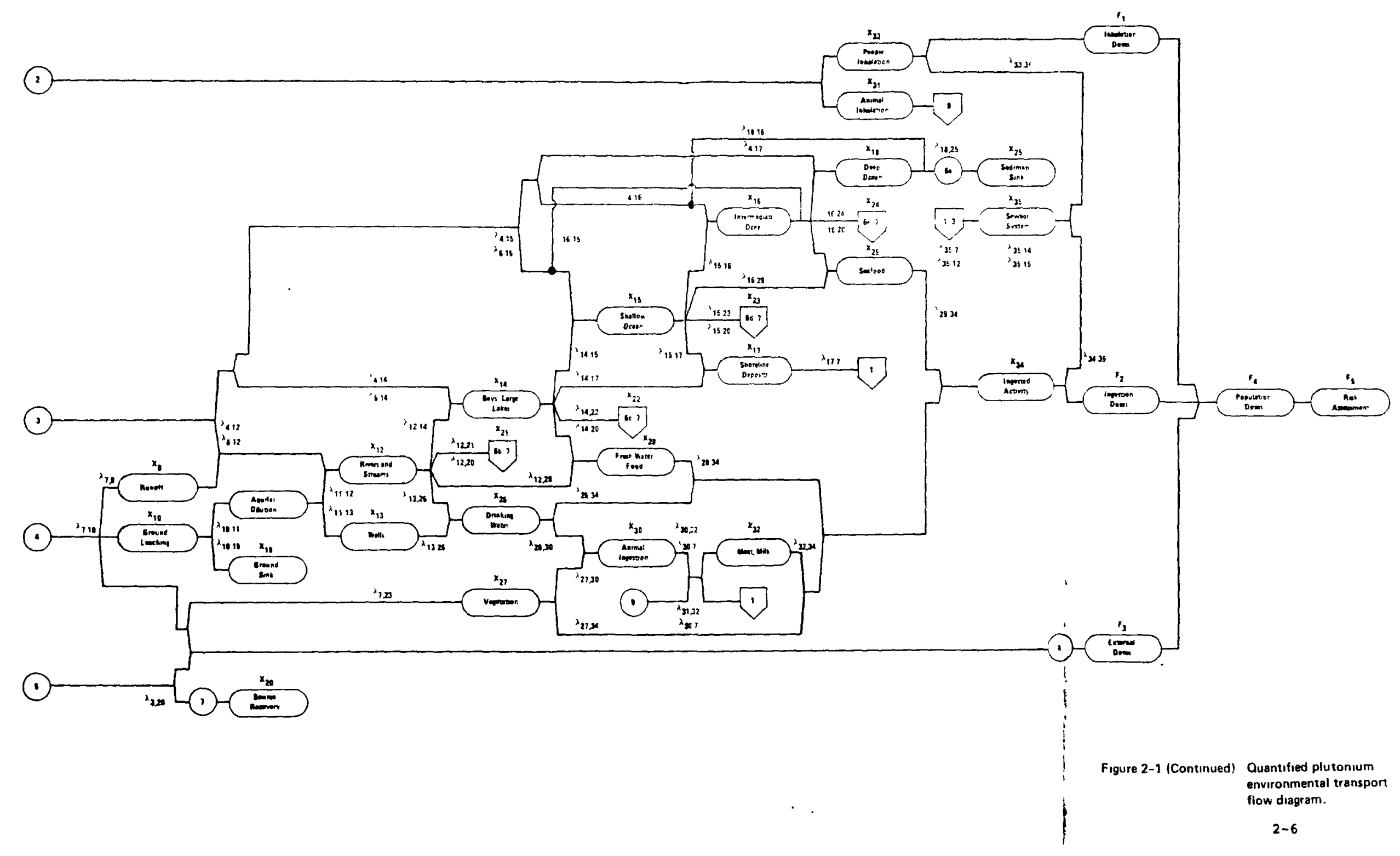



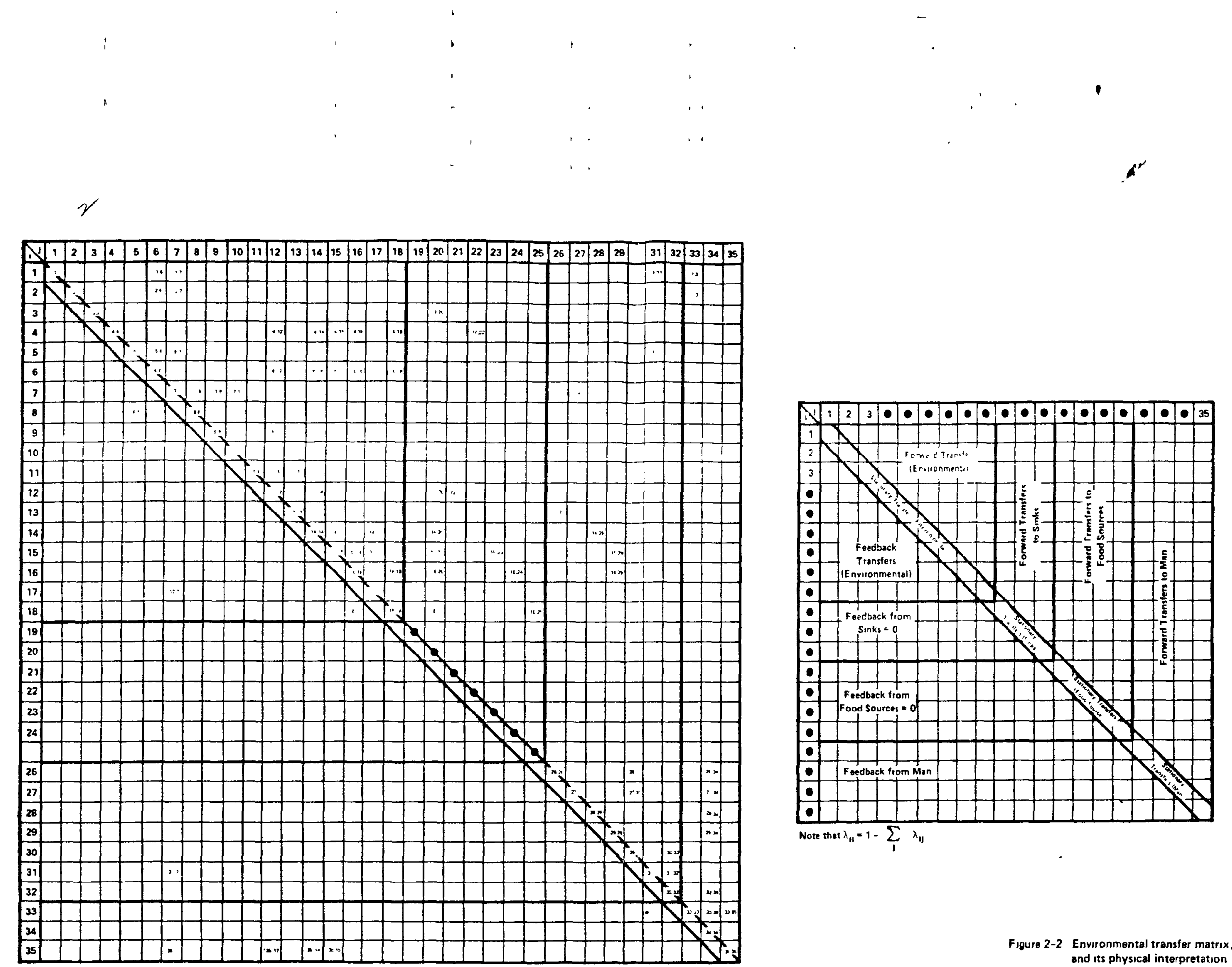

Figure 2-2 Environmental transfer matrix $\left[\lambda_{1,1}\right]$ and its physical interpretation 

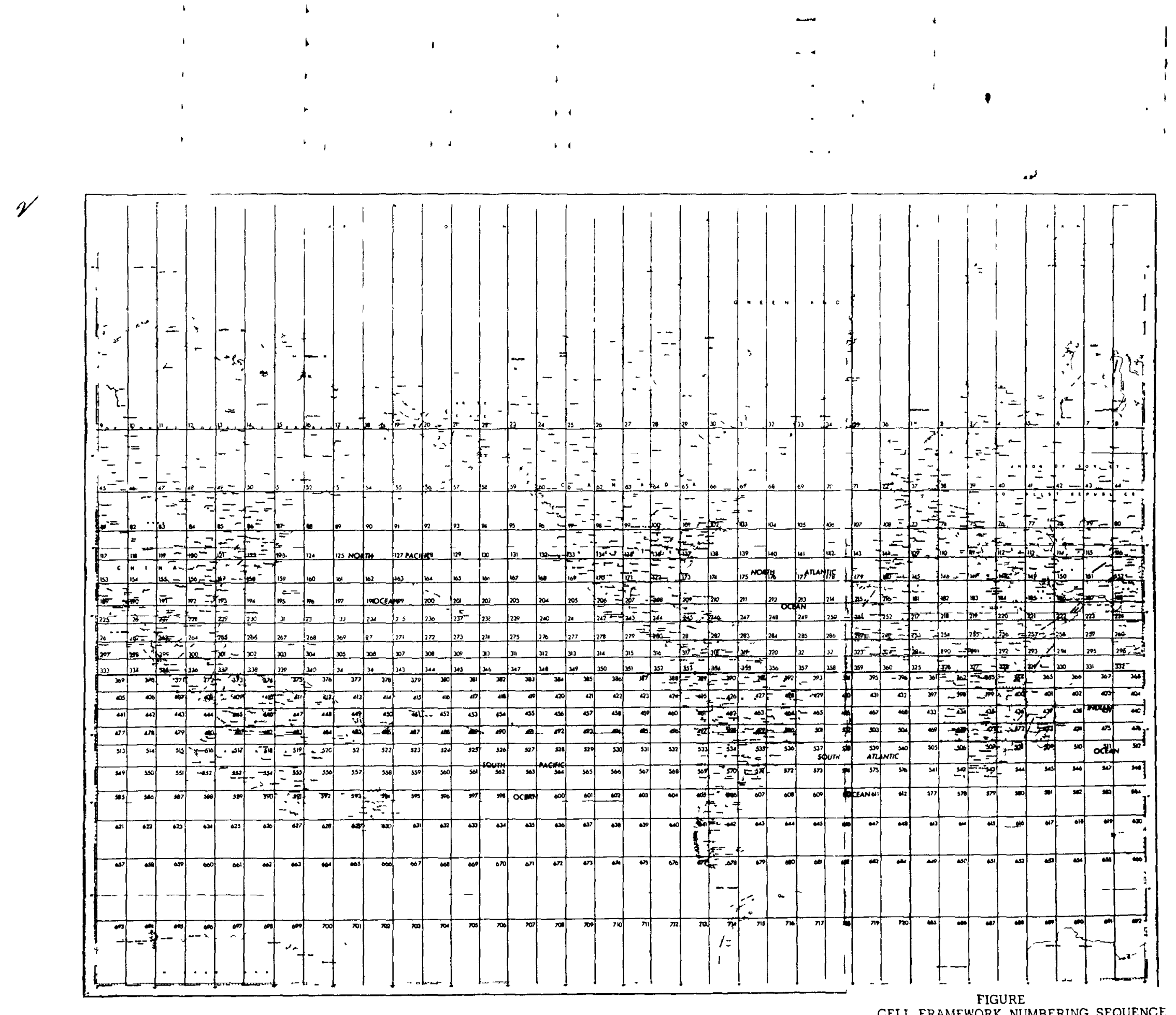